\title{
Rigidity of 3-colorings of the discrete torus
}

\author{
Ohad Noy Feldheim ${ }^{\mathrm{a}, 1}$ and Ron Peled ${ }^{\mathrm{b}, 2}$ \\ a Mathematics Department, Stanford University, Stanford, CA, USA. \\ E-mail: ohadfeld@stanford.edu \\ ${ }^{\mathrm{b}}$ School of Mathematics, Raymond and Beverly Sackler Faculty of Exact Sciences, Tel Aviv University, Tel Aviv, Israel. \\ E-mail: peledron@post.tau.ac.il
}

Received 27 May 2015; revised 14 December 2016; accepted 8 March 2017

\begin{abstract}
We prove that a uniformly chosen proper 3-coloring of the $d$-dimensional discrete torus has a very rigid structure when the dimension $d$ is sufficiently high. We show that with high probability the coloring takes just one color on almost all of either the even or the odd sub-torus. In particular, one color appears on nearly half of the torus sites. This model is the zero temperature case of the 3-state anti-ferromagnetic Potts model from statistical physics.

Our work extends previously obtained results for the discrete torus with specific boundary conditions. The main challenge in this extension is to overcome certain topological obstructions which appear when no boundary conditions are imposed on the model. Locally, a proper 3-coloring defines the discrete gradient of an integer-valued height function which changes by exactly one between adjacent sites. However, these locally-defined functions do not always yield a height function on the entire torus, as the gradients may accumulate to a non-zero quantity when winding around the torus. Our main result is that in high dimensions, a global height function is well defined with high probability, allowing to deduce the rigid structure of the coloring from previously known results. Moreover, the probability that the gradients accumulate to a vector $m$, corresponding to the winding in each of the $d$ directions, is at most exponentially small in the product of $\|m\|_{\infty}$ and the area of a cross-section of the torus.

In the course of the proof we develop discrete analogues of notions from algebraic topology. This theory is developed in some generality and may be of use in the study of other models.
\end{abstract}

Résumé. Nous montrons qu'un 3-coloriage aléatoire du tore $d$-dimensionnel a une structure rigide quand la dimension $d$ est suffisamment grande. Nous montrons qu'avec grande probabilité, le coloriage prend au plus une couleur sur, soit presque tout le sous-tore pair, soit presque tout le sous-tore impair. En particulier, une couleur apparaît sur presque la moitié des sites du tore. Ce modèle correspond au cas de température 0 du modèle de Potts anti-ferromagnétique à trois états, en physique statistique.

Notre travail étend les résultats obtenus pour le tore discret avec des conditions frontière spécifiques. La difficulté principale dans cette extension est de surmonter les obstructions topologiques qui apparaissent quand aucune condition frontière n'est imposée. Localement, un 3-coloriage propre définit le gradient discret d'une fonction de hauteur à valeurs entières qui diffère exactement d'un entre deux points voisins. Néanmoins, ces fonctions locales ne définissent pas forcément une fonction de hauteur sur le tore entier, car les gradients peuvent s'accumuler en une quantité non-nulle en s'enroulant autour du tore. Notre résultat principal est qu'en grande dimension, une fonction de hauteur est bien définie sur le tore entier avec grande probabilité, ce qui permet de déduire la structure rigide du coloriage en utilisant des résultats précédents. De plus, la probabilité que le gradient s'accumule en un vecteur $m$ correspondant à l'enroulement le long de chacune des $d$ directions est exponentiellement petit en le produit de $\|m\|_{\infty}$ et de l'aire de la section du tore dans cette direction.

Au long de la preuve, nous introduisons des analogues discrets de notions de topologie algébrique. Cette théorie est développée dans une généralité qui peut permettre une utilisation pour d'autres modèles.

MSC: Primary 82B20; 82B26; secondary 82B41; 60C05; 60D05; 60K35; $05 \mathrm{~A} 16$

\footnotetext{
${ }^{1}$ Research supported by an ERC advanced grant.

${ }^{2}$ Research supported by an ISF grant and an IRG grant.
} 


\section{Introduction}

We study proper 3 -colorings of $\mathbb{T}_{n}^{d}$, the d-dimensional discrete torus $(\mathbb{Z} / n \mathbb{Z})^{d}$, whose side length $n$ is even. Our main theorem is that in high dimensions, a uniformly chosen proper 3-coloring of $\mathbb{T}_{n}^{d}$ is nearly constant on one of the two bipartition classes of $\mathbb{T}_{n}^{d}$. Precisely, denote the partite classes of $\mathbb{T}_{n}^{d}$ by $V^{0}$ and $V^{1}$. A proper 3-coloring of $\mathbb{T}_{n}^{d}$ is a function $f: \mathbb{T}_{n}^{d} \rightarrow\{0,1,2\}$ satisfying $f(v) \neq f(w)$ whenever $v$ and $w$ are adjacent in $\mathbb{T}_{n}^{d}$. Denote by $\mathrm{CP}_{i, k}(f)$ the proportion of color $k$ on $V^{i}$, that is,

$$
\mathrm{CP}_{i, k}(f):=\frac{\left|\left\{v \in V^{i}: f(v)=k\right\}\right|}{\left|V^{i}\right|} .
$$

Theorem 1.1. There exist $d_{0}, c>0$ such that for every integer $d \geq d_{0}$ and every even integer $n$, a uniformly chosen proper 3-coloring $f: \mathbb{T}_{n}^{d} \rightarrow\{0,1,2\}$ satisfies

$$
\mathbb{E}\left(\min _{i \in\{0,1\}} \mathrm{CP}_{i, k}(f)\right) \leq \exp \left(-\frac{c d}{\log ^{2} d}\right) \text { for all } k \in\{0,1,2\} .
$$

Thus, the theorem asserts that typically in high dimensions, for each color there is a partite class on which the color hardly appears. Equivalently, one of the partite classes is dominated by a single color.

The next section describes the main idea of the proof. More precise definitions are given in Section 2.

\subsection{Relation with height functions}

Our proof of Theorem 1.1 exploits a connection between proper 3-colorings and height functions, which we now describe. It is convenient to introduce the required notions on a general graph. Suppose $G$ is a connected, bipartite graph with a fixed vertex $v_{0} \in V(G)$. Let $\operatorname{Col}\left(G, v_{0}\right)$ be the set of all proper 3-colorings of $G$ taking the value 0 at $v_{0}$. That is,

$$
\operatorname{Col}\left(G, v_{0}\right):=\left\{f: V(G) \rightarrow\{0,1,2\}: f\left(v_{0}\right)=0, f(v) \neq f(w) \text { when }(v, w) \in E(G)\right\} .
$$

An integer-valued function on $V(G)$ is called a homomorphism height function on $G$, or simply height function or HHF, if it differs by exactly one between adjacent vertices of $G$. Let $\operatorname{Hom}\left(G, v_{0}\right)$ be the set of all homomorphism height functions on $G$ which take the value 0 at $v_{0}$. Precisely,

$$
\operatorname{Hom}\left(G, v_{0}\right):=\left\{f: V(G) \rightarrow \mathbb{Z}: f\left(v_{0}\right)=0,|f(v)-f(w)|=1 \text { when }(v, w) \in E(G)\right\} .
$$

In this paper, we always take $G$ to be either $\mathbb{T}_{n}^{d}$ or $\mathbb{Z}^{d}$ for some $n$ and $d$. We consider both $\mathbb{T}_{n}^{d}$ and $\mathbb{Z}^{d}$ to come with a fixed coordinate system and denote by $\mathbf{0}$ the vector $(0,0, \ldots, 0)$ in that system. For these graphs, we abbreviate $\operatorname{Col}(G, \mathbf{0})$ to $\operatorname{Col}(G)$ and $\operatorname{Hom}(G, \mathbf{0})$ to $\operatorname{Hom}(G)$.

The connection we need between proper colorings and height functions is summarized by the following two facts:

1. For any graph $G, v_{0} \in V(G)$ and $h \in \operatorname{Hom}\left(G, v_{0}\right)$, the function $g: V(G) \rightarrow\{0,1,2\}$ defined by

$$
g(v):=h(v) \bmod 3
$$

belongs to $\operatorname{Col}\left(G, v_{0}\right)$.

2. When $G=\mathbb{Z}^{d}$, the above correspondence defines a bijection between $\operatorname{Hom}\left(\mathbb{Z}^{d}\right)$ and $\operatorname{Col}\left(\mathbb{Z}^{d}\right)$.

The first fact is straightforward and the second fact appears to be folklore in the field (see Proposition 2.1).

Our goal in this work is to use the above correspondence to transfer known results on height functions, proved in [18], to results on colorings, thereby obtaining Theorem 1.1. Our task is, however, made complicated by the following 
obstruction. The above correspondence is not a bijection when $G=\mathbb{T}_{n}^{d}$. In other words, there exist colorings in $\operatorname{Col}\left(\mathbb{T}_{n}^{d}\right)$ which are not the modulo 3 of any height function in Hom $\left(\mathbb{T}_{n}^{d}\right)$. For instance, the coloring 012012 of $\mathbb{T}_{6}^{1}$ provides one such example. The source of this problem is of a topological nature, stemming from the fact that the torus has non-contractible cycles. This poses a major difficulty, preventing a direct use of the known results on height functions. The following theorem, whose proof occupies most of this paper, provides a way around this difficulty. It shows that the above correspondence is, nonetheless, close to being bijective when the dimension $d$ is sufficiently high.

Theorem 1.2. There exist $d_{0}$ and $c>0$ such that for every integer $d \geq d_{0}$ and every even integer $n$, a uniformly chosen proper 3-coloring of $\mathbb{T}_{n}^{d}$ satisfies

$\mathbb{P}\left(f\right.$ is not the modulo 3 of some HHF on $\left.\mathbb{T}_{n}^{d}\right) \leq \exp \left(-c_{d} n^{d-1}\right)$,

with $c_{d}=\frac{c}{d \log ^{2} d}$.

In the next section we explain how Theorem 1.1 follows from the above theorem and a result on height functions proved in [18]. In Section 1.3 we present some background. The rest of the paper is devoted to the proof of Theorem 1.2. Section 2 contains the first part of the proof and a proof overview. The proof is inspired by ideas from algebraic topology but the necessary tools are developed completely in the discrete setting. We believe that some of these tools could prove useful in other models as well, especially the trichotomy theorems of Section 3, Theorem 3.2 and Theorem 3.4, which deal with discrete counterparts of manifolds of codimension one. The connection between our work and algebraic topology is expounded upon in Section 2.4. Section 8 is dedicated to remarks and open problems.

\subsection{Remarks and extensions}

We point out that the bound presented in Theorem 1.1 is near optimal. Perhaps surprisingly, Theorem 1.1 itself implies the following claim.

Proposition 1.3. There exist $d_{0}, c>0$ such that for every integer $d \geq d_{0}$ and every even integer $n$, a uniformly chosen proper 3-coloring $f: \mathbb{T}_{n}^{d} \rightarrow\{0,1,2\}$ satisfies

$$
\mathbb{E}\left(\min _{i \in\{0,1\}} \mathrm{CP}_{i, k}(f)\right) \geq \exp (-c d) \quad \text { for all } k \in\{0,1,2\} .
$$

This proposition is proved in Section 7.

We also emphasize that Theorem 1.2 serves as a bridge between results on uniformly sampled homomorphism height functions on $\mathbb{T}_{n}^{d}$ and uniformly sampled proper 3-colorings. Thus, results on the former may be transferred easily to the latter, as is illustrated by the deduction of Theorem 1.1 in Section 1.4. One expects it to be possible to upgrade Theorem 1.1 by showing that the quantity $\min _{i \in\{0,1\}} \mathrm{CP}_{i, k}(f)$ is not only small on average, but also small with high probability as $n$ tends to infinity. To use Theorem 1.2 to this end would require extending the corresponding results on height functions. While we believe such extensions are possible, we do not delve further in this direction as our main concern in this paper is to establish the relation between the models.

As explained in Section 2 below, we approach Theorem 1.2 by identifying the set of proper 3-colorings with a set of quasi-periodic height functions. Each such height function has a well-defined slope, a vector which measures the amount by which it changes when going around the torus in each direction. Homomorphism height functions on $\mathbb{T}_{n}^{d}$ can be identified with quasi-periodic height functions with zero slope. The proof of Theorem 1.2 proceeds by finding a one-to-one map between quasi-periodic functions of a given non-zero slope, and a tiny subset of the quasi-periodic functions with zero slope, see Theorem 2.3 below. In fact, more can be deduced from our techniques. As we show in Theorem 2.4, the size of the set of quasi-periodic functions with a given slope may be estimated in terms of this slope, yielding stronger bounds for steeper slopes. For instance, the chance of sampling a proper 3-coloring whose corresponding height function changes by a linear amount when going around the torus, is exponentially small in $n^{d}$ rather than the $n^{d-1}$ appearing in Theorem 1.2. 
While Theorem 1.2 is proved in high dimensions, the main ingredient in its proof, the above-mentioned one-to-one mapping of quasi-periodic height functions with a given slope to quasi-periodic height functions with zero slope, is developed in all dimensions. The part which is missing in low dimensions is a counterpart of [18, Theorem 2.8], which would show that the probability that a low-dimensional HHF on $\mathbb{T}_{n}^{d}$ has a long level line is exponentially small in this length. This result is not expected in two dimensions (see discussion in Section 1.3 below), but may be valid already in dimensions $d \geq 3$. Theorem 1.2 would immediately extend to any dimension in which this result is established. Appropriate analogs of Theorem 1.1 in dimensions $d \geq 3$ may also be valid, as the proof of Theorem 1.1 relies on Theorem 1.2 and input on the fluctuations of homomorphism height functions provided in [18] in high dimensions (see Section 1.4 below).

\subsection{Background and related works}

Our work is not the first to establish rigidity of proper 3-colorings in high dimensions. Previously, a result analogous to Theorem 1.1 in which the proper 3-coloring is sampled from the set of colorings with 'zero boundary conditions' was established in [18], and also by Galvin, Kahn, Randall and Sorkin in [7]. The restriction to such 'zero boundary conditions' makes the problem simpler from a topological point of view since it essentially removes the non-trivial cycles of $\mathbb{T}_{n}^{d}$, rendering the correspondence described in Section 1.1 into a bijection of height functions and proper 3 -colorings with these boundary conditions. The results of [18] and [7] imply Roman Kotecký's conjecture (see [14] for context and [7] for additional details), that the proper 3-coloring model admits at least 6 different Gibbs states in high dimensions.

Galvin and Randall [8, Theorem 2.1] established a related result in the same setting as Theorem 1.1. They showed that for each color $k$, with probability at least $1-\exp \left(-c_{d} n^{d-1} / \log ^{2} n\right)$, the proportions of the color on the two bipartite classes differ by at least $\rho$, where $\rho \approx 0.22$. In terms of the quantities $\mathrm{CP}_{i, k}(f)$ used in Theorem 1.1 , this means that $\left|\mathrm{CP}_{0, k}(f)-\mathrm{CP}_{1, k}(f)\right| \geq \rho$ with high probability. Taking into account that each color may appear on at most half of the vertices of the torus, this implies that $\min _{i \in\{0,1\}} \mathrm{CP}_{i, k}(f) \leq \frac{1-\rho}{2} \approx 0.39$ with high probability. In contrast, Theorem 1.1 shows that $\mathbb{E}\left(\min _{i \in\{0,1\}} \mathrm{CP}_{i, k}(f)\right) \leq \exp \left(-c d / \log ^{2}(d)\right)$, a bound which is near optimal by Proposition 1.3. As discussed in Section 1.2, we believe this bound may be shown to hold not only on average but with high probability as $n$ tends to infinity by extending the corresponding results on height functions. The techniques of [8] are rather different from ours. While we proceed by developing the topological theory of discrete height functions, the work [8] stays fully in the realm of 3-colorings.

Other related results include torpid mixing of the Glauber dynamics for proper 3-colorings of $\mathbb{T}_{n}^{d}[8]$ and the fact that homomorphism height functions have bounded range on the hypercube graph $\{0,1\}^{d}$, as proved by Kahn [11] and Galvin [5].

In statistical physics terminology, the proper 3-coloring model is the same as the zero temperature case of the antiferromagnetic 3-state Potts model. It is expected that the analog of our result continues to hold for small, positive temperature, but this remains unproven. In two dimensions, the model is equivalent to the uniform six-vertex, or square ice, model (this was pointed out by Andrew Lenard, see [16]). It is expected that the analog of Theorem 1.1 fails in two dimensions, as the square ice model is conjectured to be in a disordered phase, in the sense that the model should have a unique Gibbs state when $d=2$. However, it may well be that multiple Gibbs states exist already for any $d \geq 3$. Investigating other graphs, Kotecký, Sokal and Swart [15] have shown that the model has multiple Gibbs states on certain planar lattices. This result was extended by Huang et al. [10] who have shown that for every $q \geq 3$, there are planar lattices on which the proper $q$-coloring model has multiple Gibbs states.

The fact that a uniformly chosen 3-coloring on the torus is the modulo 3 of a height function with high probability (Theorem 1.2) is also expected to fail in two dimensions. Some evidence for this phenomenon is provided by the study of the dimer model. In the dimer model, one samples uniformly a perfect matching of an underlying graph. On suitable graphs, the perfect matching defines locally the gradient of an integer-valued height function and one may study similar questions to those studied here. Boutillier and de Tilière [1] (see also Kenyon [13, Section 4.17]) considered the dimer model on a piece of the hexagonal lattice wrapped around a torus. They showed that the random height differences accumulated when winding around the torus tend to a non-degenerate limit distribution (a discrete Gaussian-type distribution) as the side length of the torus increases.

It is conjectured that the rigidity phenomenon described by Theorem 1.1 has an analog for proper colorings with more than 3 colors. Specifically, that for any $q \geq 4$ there exists a $d_{0}(q)$ such that a uniformly sampled proper $q$ coloring of $\mathbb{T}_{n}^{d}, d \geq d_{0}(q)$, has the following structure with high probability. The colors split into two sets of sizes 
$\lfloor q / 2\rfloor$ and $\lceil q / 2\rceil$, with the even sublattice colored predominantly by colors from one set and the odd sublattice colored predominantly by colors from the other set. While this conjecture remains open, several related results have appeared. Galvin and Tetali [9], following work of Kahn [12], gave approximate counts for the number of graph homomorphisms from $d$-regular graphs to arbitrary finite graphs. Specializing to proper $q$-colorings of $\mathbb{T}_{n}^{d}$, their results support the above conjecture. Meyerovitch and Pavlov [17] analyzed, so called, axial products of shifts of finite type, a more general model than graph homomorphisms on $\mathbb{Z}^{d}$, and found explicit expressions for the limiting topological entropy of such models as $d$ tends to infinity. Their results are also in agreement with the above conjecture. Galvin and Engbers [4] established the analog of the conjecture, and more general rigidity results for graph homomorphisms, in the limit when $n$ is fixed and $d$ tends to infinity. Similar rigidity results on expander and tree graphs are established in [20,21].

Of related interest is the hard-core model in $\mathbb{T}_{n}^{d}$. In this model, one samples an independent set $I$ of $\mathbb{T}_{n}^{d}$ with probability proportional to $\lambda^{|I|}$. It is expected that there exists some $\lambda_{c}=\lambda_{c}(d)$ satisfying that, with high probability, if $\lambda>\lambda_{c}$ the sampled independent set resides predominantly in one of the two sublattices, whereas if $\lambda<\lambda_{c}$ no such structure appears. While the existence of $\lambda_{c}$ is still open (and there are examples of graphs for which it does not exist, see [2]) one may still define $\lambda_{c}^{\prime}=\lambda_{c}^{\prime}(d)$ as the infimum over $\lambda$ for which the model admits multiple Gibbs states. Dobrushin [3] proved that $\lambda_{c}^{\prime}<\infty$ in every dimension $d \geq 2$, with an upper bound growing to infinity with $d$. Galvin and Kahn [6] significantly improved this result by showing that $\lambda_{c}^{\prime}$ tends to zero with $d$. The quantitative bound obtained in [6] was further improved in [19]. The main technical ingredient in both [6,19], as well as the aforementioned [7,18], is a careful analysis of the structure of certain special cutsets in $\mathbb{T}_{n}^{d}$, when the dimension $d$ is sufficiently high. This is in contrast to this work, in which discrete analogs of topological considerations constitute the bulk of the argument.

\subsection{Proof of Theorem 1.1}

We end the introduction by explaining how to deduce Theorem 1.1 from Theorem 1.2 and a result of [18] on the fluctuations of typical homomorphism height functions on $\mathbb{T}_{n}^{d}$.

We start with the following lemma, which states the required result on the typical behavior of height functions.

Lemma 1.4. There exist $c>0$ and $d_{0}$ such that in all dimensions $d \geq d_{0}$, if $h$ is uniformly sampled from $\operatorname{Hom}\left(\mathbb{T}_{n}^{d}\right)$ then

$$
\mathbb{P}(|h(u)-h(v)| \geq 3) \leq \exp \left(-\frac{c d}{\log ^{2} d}\right) \quad \forall u, v \in \mathbb{T}_{n}^{d} .
$$

Proof. Theorem 2.1 in [18] gives, in particular, that there exist $c>0$ and $d_{0}$ such that in all dimensions $d \geq d_{0}$ and for every $u, v \in \mathbb{T}_{n}^{d}$, if $h$ is uniformly sampled from $\operatorname{Hom}\left(\mathbb{T}_{n}^{d}, u\right)$, then

$$
\mathbb{P}(|h(v)| \geq 3) \leq \exp \left(-\frac{c d}{\log ^{2} d}\right) .
$$

The lemma follows from this by using the fact that the mapping $T_{u}: \operatorname{Hom}\left(\mathbb{T}_{n}^{d}\right) \rightarrow \operatorname{Hom}\left(\mathbb{T}_{n}^{d}, u\right)$ defined by $T_{u}(h)(v):=$ $h(v)-h(u)$ is a bijection.

We are now ready to prove Theorem 1.1. First, observe that by symmetry, it suffices to prove the theorem for a uniformly chosen coloring in $\operatorname{Col}\left(\mathbb{T}_{n}^{d}\right)$, i.e., a coloring normalized at $\mathbf{0}$.

Let $f$ be uniformly chosen from $\operatorname{Col}\left(\mathbb{T}_{n}^{d}\right)$. Recall that

$$
\mathrm{CP}_{i, k}(f)=\frac{\left|\left\{v \in V^{i}: f(v)=k\right\}\right|}{\left|V^{i}\right|},
$$

where $V^{0}$ and $V^{1}$ are the partite classes of $\mathbb{T}_{n}^{d}$. Fix $k \in\{0,1,2\}$ and let

$$
X:=\min _{i \in\{0,1\}} \mathrm{CP}_{i, k} .
$$

We need to show that $\mathbb{E}(X) \leq \exp \left(-c d / \log ^{2} d\right)$ for some $c>0$ and all sufficiently high $d$. 
Fix $d$ sufficiently high and $c>0$ sufficiently small for the following arguments. Define the event

$$
A:=\left\{f \text { is the modulo } 3 \text { of some HHF in } \operatorname{Hom}\left(\mathbb{T}_{n}^{d}\right)\right\} .
$$

By symmetry again, Theorem 1.2 implies that

$$
\mathbb{P}\left(A^{c}\right) \leq \exp \left(-\frac{c}{d \log ^{2} d} n^{d-1}\right)
$$

Hence,

$$
\mathbb{E}(X)=\mathbb{E}\left(X \mathbb{1}_{A}\right)+\mathbb{E}\left(X \mathbb{1}_{A^{c}}\right) \leq \mathbb{E}(X \mid A)+\exp \left(-\frac{c}{d \log ^{2} d} n^{d-1}\right) .
$$

Thus we focus on estimating $\mathbb{E}(X \mid A)$. Conditioning on $A$, there exists some $h \in \operatorname{Hom}\left(\mathbb{T}_{n}^{d}\right)$ for which $f \equiv h(\bmod 3)$. Moreover, since distinct functions in $\operatorname{Hom}\left(\mathbb{T}_{n}^{d}\right)$ give rise to distinct colorings in $\operatorname{Col}\left(\mathbb{T}_{n}^{d}\right)$ under the modulo 3 operation, it follows that, conditioned on $A, h$ is uniformly distributed in $\operatorname{Hom}\left(\mathbb{T}_{n}^{d}\right)$.

Now note that if $u, v \in \mathbb{T}_{n}^{d}$ are vertices in different partite classes of $\mathbb{T}_{n}^{d}$ then $h(u)$ and $h(v)$ have different parity. Thus, for such vertices, we have the following containment of events,

$$
\{f(u)=f(v)\}=\{h(u) \equiv h(v) \quad(\bmod 3)\} \subseteq\{|h(u)-h(v)| \geq 3\} .
$$

We conclude that $X$ satisfies the following relation.

$$
\begin{aligned}
X^{2} & =\frac{1}{\left|V^{0}\right|^{2}} \min _{i \in\{0,1\}}\left|\left\{v \in V^{i}: f(v)=k\right\}\right|^{2} \leq \frac{1}{\left|V^{0}\right|^{2}}\left|\left\{v \in V^{0}: f(v)=k\right\}\right| \cdot\left|\left\{v \in V^{1}: f(v)=k\right\}\right| \\
& \leq \frac{1}{\left|V^{0}\right|^{2}} \sum_{u \in V^{0}, v \in V^{1}} \mathbb{1}_{(f(u)=f(v))} \leq \frac{1}{\left|V^{0}\right|^{2}} \sum_{u \in V^{0}, v \in V^{1}} \mathbb{1}_{(|h(u)-h(v)| \geq 3) .} .
\end{aligned}
$$

Hence, we may use Lemma 1.4 to deduce that

$$
\mathbb{E}(X \mid A) \leq \sqrt{\mathbb{E}\left(X^{2} \mid A\right)} \leq \frac{1}{\left|V^{0}\right|} \sqrt{\sum_{u \in V^{0}, v \in V^{1}} \mathbb{P}(|h(u)-h(v)| \geq 3)} \leq \exp \left(-\frac{c d}{\log ^{2} d}\right) .
$$

Together with (1.3), this establishes Theorem 1.1.

\section{Preliminaries and overview}

This section is divided into an introduction to the objects and notation of the paper, and to a reduction of Theorem 1.2 to a statement concerning quasi-periodic functions on the integer lattice. At the end of the section we give a glimpse into the ideas of the proof, and discuss the relation between our work and algebraic topology.

\subsection{Preliminary definitions}

\section{Lattice and torus}

We write $\mathbb{Z}^{d}$ for the nearest-neighbor graph of the standard $d$-dimensional integer lattice, and $\mathbb{T}_{n}^{d}=(\mathbb{Z} / n \mathbb{Z})^{d}$ for the graph of the $d$-dimensional discrete torus with side length $n$. We assume $n$ is an even integer greater or equal than 4 , fixing it throughout the paper. We also assume both graphs come with a fixed coordinate system, letting $e_{i} \in \mathbb{Z}^{d}$ be the $i$ th standard basis vector for $1 \leq i \leq d$. In both graphs, two vertices are adjacent if they differ by one in exactly one coordinate. As $n$ is even, both graphs are bipartite. In both we thus refer to the vertices in the bipartition class of $\mathbf{0}=(0, \ldots, 0)$ as even, and to the rest of the vertices as $o d d$. For a vector $v \in \mathbb{Z}^{d}$, and a set $U \in \mathbb{Z}^{d}$ we write $U+v$ to denote $\{u+v: u \in U\}$. 
Distance and boundary

Let $G$ be a connected graph. We write $u \sim v$ to denote that a pair of vertices $u, v \in V(G)$ are adjacent. For a set of vertices $U \subseteq V(G)$ we define the boundary of $U$ to be the set of edges

$$
\partial U:=\left\{e \in E(G): e \cap U \neq \varnothing \text { and } e \cap U^{c} \neq \varnothing\right\} .
$$

We use $\operatorname{dist}(u, v)$ for the shortest-path distance between $u$ and $v$, and extend this notion to non-empty sets $U, V \subseteq$ $V(G)$, defining

$$
\operatorname{dist}(U, V):=\min \{\operatorname{dist}(u, v): u \in U, v \in V\} .
$$

If one of the sets $U, V$ is empty, we write $\operatorname{dist}(U, V)=\infty$. For a set of vertices $U$, we denote

$$
\begin{aligned}
& U^{+}:=\{u \in V(G): \operatorname{dist}(\{u\}, U) \leq 1\}, \\
& U^{-}:=\left\{u \in V(G): \operatorname{dist}\left(\{u\}, U^{c}\right)>1\right\} .
\end{aligned}
$$

Note that $U^{-}=\left(\left(U^{c}\right)^{+}\right)^{c}$. We also abbreviate $U^{++}:=\left(U^{+}\right)^{+}$and $U^{--}:=\left(U^{-}\right)^{-}$. The following simple relations hold for any two sets $U, V \subseteq V(G)$ :

$$
\begin{aligned}
& U^{+} \subseteq V \quad \Longleftrightarrow \quad U \subseteq V \quad \text { and } \quad \partial U \cap \partial V=\varnothing, \\
& \operatorname{dist}\left(U^{+}, V\right)=\max (\operatorname{dist}(U, V)-1,0), \\
& U \subseteq V \quad \Longleftrightarrow \quad \forall W \subset V(G), \quad \operatorname{dist}(U, W) \geq \operatorname{dist}(V, W) .
\end{aligned}
$$

For a set of vertices $U$, we define the internal vertex boundary of $U$ to be

$$
\partial_{\bullet} U:=U \backslash U^{-} .
$$

Similarly we define the external vertex boundary of $U$ to be

$$
\partial_{\circ} U:=U^{+} \backslash U \text {. }
$$

In both $\mathbb{Z}^{d}$ and $\mathbb{T}_{n}^{d}$, we call a set of vertices $U$ odd if all the vertices of $\partial_{\bullet} U$ have the same parity (in [18] a different convention is used, calling a set $U$ odd if all vertices of $\partial_{\bullet} U$ are odd). The internal and external vertex boundaries of an odd set of vertices $U \subsetneq T_{10}^{2}$, as well as $U^{+}$and $U^{-}$, are depicted in Figure 1 .

Homomorphism height functions, 3-colorings and quasi-periodic functions

A proper 3-coloring of a graph $G$ is a function $f: V(G) \rightarrow\{0,1,2\}$ satisfying $f(v) \neq f(w)$ when $(v, w) \in E(G)$. An integer-valued function on $V(G)$ is called a homomorphism height function on $G$, or simply height function or HHF, if it differs by exactly one between adjacent vertices of $G$. We usually work with $\operatorname{Col}\left(G, v_{0}\right)$ and $\operatorname{Hom}\left(G, v_{0}\right)$, the sets of colorings and height functions normalized to take the value 0 at the vertex $v_{0}$, as defined in (1.1) and (1.2). When $G=\mathbb{T}_{n}^{d}$ or $\mathbb{Z}^{d}$ we abbreviate $\operatorname{Col}(G, \mathbf{0})$ to $\operatorname{Col}(G)$ and $\operatorname{Hom}(G, \mathbf{0})$ to $\operatorname{Hom}(G)$.

Let $V$ be either $\mathbb{Z}$ or $\{0,1,2\}$. We say that a function

$$
f: \mathbb{Z}^{d} \rightarrow V \text { is periodic if } f(v)=f(w) \text { whenever } v-w=n e_{i} \text { for some } i .
$$

We denote by PC the set of periodic proper 3-colorings in $\operatorname{Col}\left(\mathbb{Z}^{d}\right)$. Similarly, for an integer vector $m=$ $\left(m_{1}, \ldots, m_{d}\right) \in \mathbb{Z}^{d}$, we say that a function

$$
h: \mathbb{Z}^{d} \rightarrow \mathbb{Z} \text { is quasi-periodic with slope } m \text { if } f(v)=f(w)+m_{i} \text { whenever } v-w=n e_{i} \text { for some } i .
$$

We write $\mathrm{QP}_{m}$ for the set of quasi-periodic HHFs with slope $m$ in $\operatorname{Hom}\left(\mathbb{Z}^{d}\right)$. Note that for an HHF, being periodic is equivalent to being quasi-periodic with slope $\mathbf{0}$. We remark that our definition of slope is not completely standard and it may be equally natural to say that a quasi-periodic function with slope $m$, according to our definition, has, in fact, 


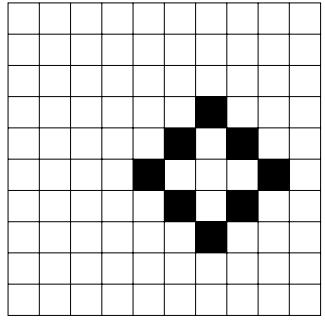

$U^{-}$

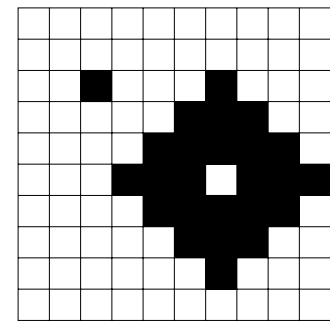

$U$

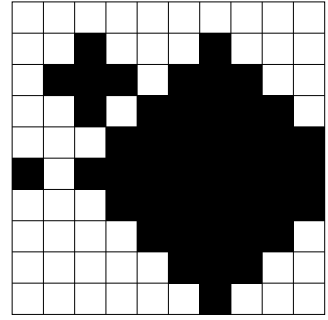

$U^{+}$

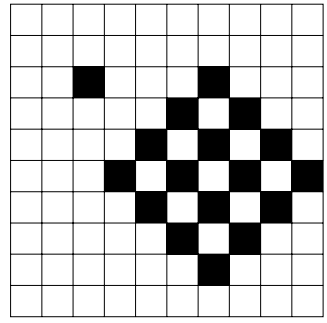

$\partial_{\bullet} U$

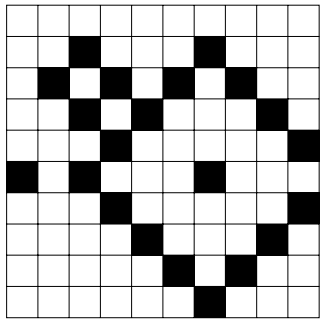

$\partial_{\circ} U$

Fig. 1. Boundary operations on some odd set $U$ in $T_{10}^{2}$.

slope $\frac{1}{n} \cdot m$. Our definition is chosen as it is convenient to work with integer vectors, keeping in mind that $n$ is fixed throughout the paper.

Observe that, in fact,

$$
\mathrm{QP}_{m}=\varnothing \text { if } m \notin 2 \mathbb{Z}^{d} \text { or if }\left|m_{i}\right|>n \text { for some } i .
$$

To see this, note that any $h \in \operatorname{Hom}\left(\mathbb{Z}^{d}\right)$ must take even values on even vertices, and satisfy $|h(v)| \leq \operatorname{dist}(v, 0)$, since $h$ changes by one between adjacent vertices. Thus, we must have that $m_{i}=h\left(n e_{i}\right)$ is even and $\left|h\left(n e_{i}\right)\right| \leq n$ for all $i$. The quasi-periodic functions whose slope is not a multiple of 6 will not play a role in our work, as we show in Proposition 2.2. Thus we define

$$
\mathrm{QP}:=\bigcup_{m \in 6 \mathbb{Z}^{d} \cap[-n, n]^{d}} \mathrm{QP}_{m} .
$$

Denote by $\pi: \mathbb{Z}^{d} \rightarrow \mathbb{T}_{n}^{d}$ the natural projection from the integer lattice to the torus, defined by

$$
\pi\left(\left(v_{1}, \ldots, v_{d}\right)\right)=\left(v_{1} \bmod n, \ldots, v_{d} \bmod n\right)
$$

(where we identify the coordinate system of the torus with $\{0, \ldots, n-1\}^{d}$ ). Observe that $\pi$ extends naturally to a bijection between periodic proper 3-colorings (of $\mathbb{Z}^{d}$ ) and proper 3-colorings of $\mathbb{T}_{n}^{d}$, as well as to a bijection between periodic HHFs (on $\mathbb{Z}^{d}$ ) and HHFs on $\mathbb{T}_{n}^{d}$. With a slight abuse of notation we also denote these extensions by $\pi$.

\section{Relations between HHFs and 3-colorings}

It is not difficult to see that the mapping $\operatorname{Mod}_{3}$, which takes an HHF $h$ to the function defined by

$$
\operatorname{Mod}_{3}(h)(v):=h(v) \bmod 3,
$$

maps every HHF to a proper 3-coloring. As mentioned in the introduction, it is a known fact that $\operatorname{Mod}_{3}$ defines a bijection between $\operatorname{Hom}\left(\mathbb{Z}^{d}\right)$ and $\operatorname{Col}\left(\mathbb{Z}^{d}\right)$, that is between the set of HHFs on $\mathbb{Z}^{d}$ normalized at $\mathbf{0}$ and the set of proper 3-colorings of $\mathbb{Z}^{d}$ normalized at $\mathbf{0}$. As we could not locate a reference for this fact, we provide a short proof now. 
Proposition 2.1. The map $\operatorname{Mod}_{3}$ defines a bijection between $\operatorname{Hom}\left(\mathbb{Z}^{d}\right)$ and $\operatorname{Col}\left(\mathbb{Z}^{d}\right)$.

Proof. We first check that $\operatorname{Mod}_{3}$ is an injective map. Suppose $h_{1}, h_{2} \in \operatorname{Hom}\left(\mathbb{Z}^{d}\right)$ are two distinct height functions with $\operatorname{Mod}_{3}\left(h_{1}\right)=\operatorname{Mod}_{3}\left(h_{2}\right)$. As $h_{1}(\mathbf{0})=h_{2}(\mathbf{0})=0$, it follows that there exist two adjacent vertices $v, w \in \mathbb{Z}^{d}$ satisfying that $h_{1}(v)=h_{2}(v)$ but $h_{1}(w) \neq h_{2}(w)$. However, as $\left|h_{1}(v)-h_{1}(w)\right|=\left|h_{2}(v)-h_{2}(w)\right|=1$, this contradicts our assumption that $\operatorname{Mod}_{3}\left(h_{1}\right)(w)=\operatorname{Mod}_{3}\left(h_{2}\right)(w)$.

We proceed to show that $\operatorname{Mod}_{3}$ is onto. Let $f \in \operatorname{Col}\left(\mathbb{Z}^{d}\right)$. Our goal is to define an $h \in \operatorname{Hom}\left(\mathbb{Z}^{d}\right)$ satisfying that $\operatorname{Mod}_{3}(h)=f$. First, define a spanning tree $\mathcal{T}$ of $\mathbb{Z}^{d}$, rooted at $\mathbf{0}$, as follows: Given $v=\left(v_{1}, \ldots, v_{d}\right) \in \mathbb{Z}^{d} \backslash\{\mathbf{0}\}$ let $k(v)$ equal the minimal $k$ for which $v_{k} \neq 0$. Define the parent $v^{*}$ of $v$ in $\mathcal{T}$ by setting $v_{j}^{*}=v_{j}$ for all $j \neq k(v)$ and setting $v_{k(v)}^{*}=v_{k(v)}-1$ if $v_{k(v)}>0$ or $v_{k(v)}^{*}=v_{k(v)}+1$ if $v_{k(v)}<0$, noting that $v^{*} \sim v$ and $\operatorname{dist}\left(v^{*}, \mathbf{0}\right)=\operatorname{dist}(v, \mathbf{0})-1$. Now define $h(v)$ by induction on $\operatorname{dist}(v, \mathbf{0})$. Set $h(\mathbf{0}):=0$ and, for $v \in \mathbb{Z}^{d} \backslash\{\mathbf{0}\}$,

set $h(v)$ to be the unique integer satisfying $\left|h(v)-h\left(v^{*}\right)\right|=1$ and $h(v) \equiv f(v) \quad(\bmod 3)$.

As we clearly have $\operatorname{Mod}_{3}(h)=f$, it remains to verify that $h \in \operatorname{Hom}\left(\mathbb{Z}^{d}\right)$

Let $v, w \in \mathbb{Z}^{d}$ be adjacent vertices. We need to show that

$$
|h(v)-h(w)|=1 .
$$

Assume without loss of generality that $\operatorname{dist}(v, \mathbf{0})=\operatorname{dist}(w, \mathbf{0})+1$. We proceed again by induction on $\operatorname{dist}(v, \mathbf{0})$. If $w=\mathbf{0}$ or $v_{j} \neq w_{j}$ for some $j \leq k(w)$ then necessarily $v^{*}=w$, whence (2.7) follows from (2.6). Otherwise, observe that $v^{*} \sim w^{*}$. By the induction assumption, $\left|h\left(v^{*}\right)-h\left(w^{*}\right)\right|=1$. Using also the fact that $\left|h(v)-h\left(v^{*}\right)\right|=$ $\left|h(w)-h\left(w^{*}\right)\right|=1$ and $h(v) \not \equiv h(w)(\bmod 3)$ by $(2.6)$, it follows that (2.7) holds, as required.

This bijection does not extend to $\mathbb{T}_{n}^{d}$, as there are colorings in $\operatorname{Col}\left(\mathbb{T}_{n}^{d}\right)$ which are not the image of any HHF through $\operatorname{Mod}_{3}$. Nonetheless, $\operatorname{Col}\left(\mathbb{T}_{n}^{d}\right)$ is still in bijection with a subclass of quasi-periodic functions, as the following proposition states.

Proposition 2.2. The mapping $\pi \circ \operatorname{Mod}_{3}: \mathrm{QP} \rightarrow \operatorname{Col}\left(\mathbb{T}_{n}^{d}\right)$ is a bijection.

Proof. We first show that the mapping is well-defined. Let $h \in \mathrm{QP}_{m}$ for some $m \in 6 \mathbb{Z}^{d}$. By quasi-periodicity, $h(v) \equiv$ $h\left(v+n e_{i}\right)(\bmod 3)$, for all $1 \leq i \leq d$ and $v \in \mathbb{Z}^{d}$. Consequently $\operatorname{Mod}_{3}(h) \in \mathrm{PC}$ and hence $\pi$ may be applied to $\operatorname{Mod}_{3}(h)$ to produce an element of $\operatorname{Col}\left(\mathbb{T}_{n}^{d}\right)$.

Since $\operatorname{Mod}_{3}$ is a bijection between $\operatorname{Hom}\left(\mathbb{Z}^{d}\right)$ and $\operatorname{Col}\left(\mathbb{Z}^{d}\right)$ and $\pi$ is a bijection between PC and $\operatorname{Col}\left(\mathbb{T}_{n}^{d}\right)$, we deduce that $\pi \circ \operatorname{Mod}_{3}$ is one-to-one on QP. All that remains in order to show that this mapping is a bijection, is to prove that it is onto.

Let $f \in \operatorname{Col}\left(\mathbb{T}_{n}^{d}\right)$. Define $g:=\pi^{-1}(f) \in \mathrm{PC}$ and an HHF $h$ by $h:=\operatorname{Mod}_{3}^{-1}(g)$. We need to show that $h \in \mathrm{QP}_{m}$ for some $m \in 6 \mathbb{Z}^{d} \cap[-n, n]^{d}$. We first show that for any $v, w \in \mathbb{Z}^{d}$ and $1 \leq i \leq d$,

$$
h\left(v+n e_{i}\right)-h(v)=h\left(w+n e_{i}\right)-h(w) .
$$

For this it suffices to show that for any $v \in \mathbb{Z}^{d}$ and $1 \leq i, j \leq d$,

$$
h\left(v+n e_{i}\right)-h(v)=h\left(v+e_{j}+n e_{i}\right)-h\left(v+e_{j}\right) .
$$

Since $h\left(v+e_{j}\right)-h(v)$ and $h\left(v+e_{j}+n e_{i}\right)-h\left(v+n e_{i}\right)$ are both in $\{-1,1\}$ by the definition of homomorphism height function, the equality (2.8) follows upon recalling that $g=\operatorname{Mod}_{3}(h)$ and noting that

$$
g\left(v+e_{j}\right)-g(v)=g\left(v+e_{j}+n e_{i}\right)-g\left(v+n e_{i}\right),
$$

since $g$ is periodic. Thus $h \in \mathrm{QP}_{m}$ for some $m \in \mathbb{Z}^{d}$.

It remains to show that $m \in 6 \mathbb{Z}^{d} \cap[-n, n]^{d}$. By (2.4) it suffices to show that $m \in 3 \mathbb{Z}^{d}$. This follows from the fact that

$$
m_{i}=h\left(n e_{i}\right) \equiv g\left(n e_{i}\right)=g(\mathbf{0})=0 \quad(\bmod 3) .
$$




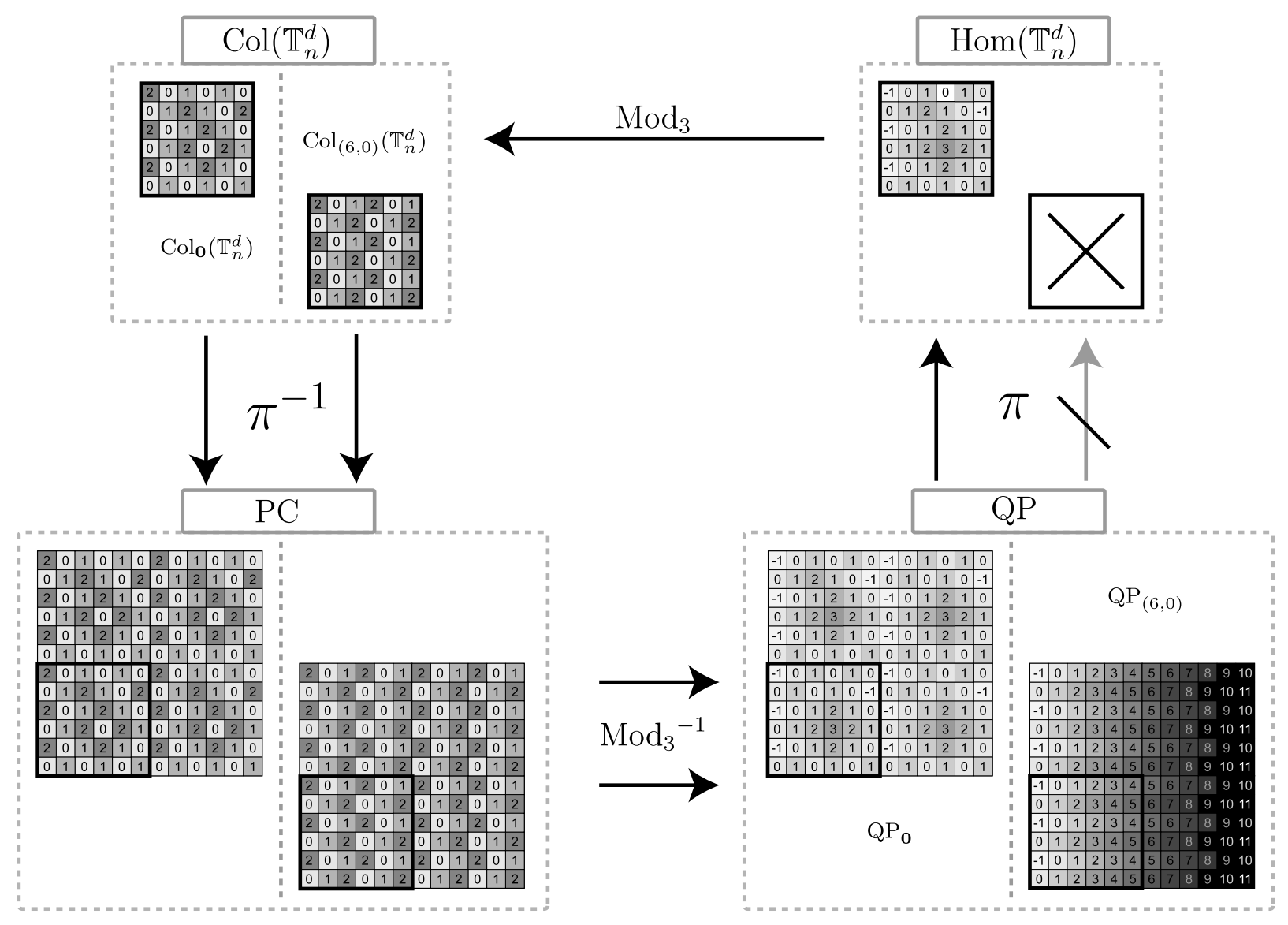

Fig. 2. The relations between $\operatorname{Col}\left(\mathbb{T}_{n}^{d}\right)$ and $\operatorname{Hom}\left(\mathbb{T}_{n}^{d}\right)$ through periodic colorings and quasi-periodic HHFs on $\mathbb{Z}^{d}$. Notice that for PC and QP only a small region of the infinite lattice is illustrated. All functions are normalized at $\mathbf{0}$, at the lower left corner of the displayed region. The illustrations depict the case $n=6, d=2$.

Proposition 2.2 enables us to define the following partition of $\operatorname{Col}\left(\mathbb{T}_{n}^{d}\right)$,

$$
\operatorname{Col}_{m}\left(\mathbb{T}_{n}^{d}\right):=\left(\pi \circ \operatorname{Mod}_{3}\right)\left(\mathrm{QP}_{m}\right) .
$$

It also implies the important fact that $\operatorname{Col}_{0}\left(\mathbb{T}_{n}^{d}\right)$ and $\operatorname{Hom}\left(\mathbb{T}_{n}^{d}\right)$ are in bijection via $\pi \circ \operatorname{Mod}_{3}^{-1} \circ \pi^{-1}$. In other words,

$$
\operatorname{Col}_{\mathbf{0}}\left(\mathbb{T}_{n}^{d}\right)=\left\{f \in \operatorname{Col}\left(\mathbb{T}_{n}^{d}\right): f \text { is the modulo } 3 \text { of some } h \in \operatorname{Hom}\left(\mathbb{T}_{n}^{d}\right)\right\}
$$

The relations between $\operatorname{Col}\left(\mathbb{T}_{n}^{d}\right), \operatorname{Hom}\left(\mathbb{T}_{n}^{d}\right), \mathrm{QP}$ and $\mathrm{PC}$ are summarized in Figure 2.

\subsection{Most elements of $\mathrm{QP}$ are in $\mathrm{QP}_{\mathbf{0}}$}

The following theorem states that most elements of QP have slope $\mathbf{0}$. This is equivalent to stating that most elements of $\operatorname{Col}\left(\mathbb{T}_{n}^{d}\right)$ are in $\operatorname{Col}_{0}\left(\mathbb{T}_{n}^{d}\right)$.

Theorem 2.3. There exist $d_{0}$ and $c>0$ such that in all dimensions $d \geq d_{0}$, for every $m \in 6 \mathbb{Z}^{d} \backslash\{\mathbf{0}\}$ we have

$$
\frac{\left|\mathrm{QP}_{m}\right|}{\left|\mathrm{QP}_{\mathbf{0}}\right|} \leq \exp \left(-c_{d} n^{d-1}\right)
$$

with $c_{d}=\frac{c}{d \log ^{2} d}$. 
Our techniques also allow us to obtain a stronger version of Theorem 2.3. This version is not required for the proof of Theorem 1.2, but is of independent interest as it significantly improves the bound on the size of $\mathrm{QP}_{m}$ when $m$ has a large coordinate. For clarity of presentation, most of the paper is devoted to the proof of Theorem 2.3 and the necessary modifications required to obtain Theorem 2.4 are then detailed in Section 6.

Theorem 2.4. There exist $d_{0}$ and $c>0$ such that in all dimensions $d \geq d_{0}$, for every $m \in 6 \mathbb{Z}^{d} \backslash\{\mathbf{0}\}$ we have

$$
\frac{\left|\mathrm{QP}_{m}\right|}{\left|\mathrm{QP}_{\mathbf{0}}\right|} \leq \exp \left(-c_{d} n^{d-1} \cdot \max _{1 \leq i \leq d}\left|m_{i}\right|\right)
$$

with $c_{d}=\frac{c}{d \log ^{2} d}$.

In thermodynamic terms, a consequence of this theorem is that the surface tension is non-differentiable at $\mathbf{0}$ as a function of the normalized slope $s=\frac{1}{n} \cdot m$. More precisely, for each $s \in \mathbb{R}^{d}$, the theorem implies that

$$
\limsup _{n \rightarrow \infty} \frac{1}{\left|\mathbb{T}_{n}^{d}\right|} \log \left(\frac{\left|\operatorname{Col}_{\lfloor s \cdot n\rfloor}\left(\mathbb{T}_{n}^{d}\right)\right|}{\left|\operatorname{Col}\left(\mathbb{T}_{n}^{d}\right)\right|}\right)=\limsup _{n \rightarrow \infty} \frac{1}{\left|\mathbb{T}_{n}^{d}\right|} \log \left(\frac{\left|\mathrm{QP}_{\lfloor s \cdot n\rfloor}\right|}{|\mathrm{QP}|}\right) \leq-c_{d} \max _{1 \leq i \leq d}\left|s_{i}\right|
$$

while

$$
\lim _{n \rightarrow \infty} \frac{1}{\left|\mathbb{T}_{n}^{d}\right|} \log \left(\frac{\left|\operatorname{Col}_{\mathbf{0}}\left(\mathbb{T}_{n}^{d}\right)\right|}{\left|\operatorname{Col}\left(\mathbb{T}_{n}^{d}\right)\right|}\right)=\lim _{n \rightarrow \infty} \frac{1}{\left|\mathbb{T}_{n}^{d}\right|} \log \left(\frac{\left|\mathrm{QP}_{\mathbf{0}}\right|}{|\mathrm{QP}|}\right)=0 .
$$

Given (2.5), we observe that Theorem 2.3 and Theorem 2.4 are trivial for $n \leq 4$, as in those cases $\mathrm{QP}_{m}$ is empty for $m \neq \mathbf{0}$. Thus, we shall assume $n \geq 6$ in the proofs of these theorems.

Theorem 1.2 is an immediate consequence of (and is, in fact, equivalent to) Theorem 2.3.

Proof of Theorem 1.2 from Theorem 2.3. By symmetry, it is enough to prove Theorem 1.2 for colorings normalized at $\mathbf{0}$. That is, to establish that for sufficiently large $d$, if $f$ is uniformly sampled from $\operatorname{Col}\left(\mathbb{T}_{n}^{d}\right)$ then

$$
\mathbb{P}\left(f \text { is not the modulo } 3 \text { of some } h \in \operatorname{Hom}\left(\mathbb{T}_{n}^{d}\right)\right) \leq \exp \left(-\frac{c}{d \log ^{2} d} n^{d-1}\right) .
$$

Suppose then that $f$ is uniformly sampled from $\operatorname{Col}\left(\mathbb{T}_{n}^{d}\right)$. By Proposition 2.2, (2.5), (2.9) and (2.10),

$$
\begin{aligned}
\mathbb{P}\left(f \text { is not the modulo } 3 \text { of some } h \in \operatorname{Hom}\left(\mathbb{T}_{n}^{d}\right)\right) & =\frac{\left|\bigcup_{m \in\left(6 \mathbb{Z}^{d} \cap[-n, n]^{d}\right) \backslash\{\mathbf{0}\}} \operatorname{Col}_{m}\left(\mathbb{T}_{n}^{d}\right)\right|}{\left|\operatorname{Col}\left(\mathbb{T}_{n}^{d}\right)\right|} \\
& =\frac{\left|\bigcup_{m \in\left(6 \mathbb{Z}^{d} \cap[-n, n]^{d}\right) \backslash\{\mathbf{0}\}} \mathrm{QP}_{m}\right|}{|\mathrm{QP}|} \leq(2 n+1)^{d} \max _{m \in 6 \mathbb{Z}^{d} \backslash\{\mathbf{0}\}} \frac{\left|\mathrm{QP}_{m}\right|}{\left|\mathrm{QP}_{\mathbf{0}}\right|}
\end{aligned}
$$

Thus (2.13) follows from Theorem 2.3.

\subsection{Proof overview}

Most of the remainder of the paper is dedicated to proving Theorem 2.3. Our proof can be divided into two parts. First we construct a set of one-to-one mappings, $\Psi_{m}: \mathrm{QP}_{m} \rightarrow \mathrm{QP}_{\mathbf{0}}$ for $m \in 6 \mathbb{Z}^{d} \backslash\{\mathbf{0}\}$. We then apply results from [18] to show that the image of $\mathrm{QP}_{m}$ under $\Psi_{m}$ is relatively small. Theorem 2.3 follows. In this section we present for the reader a rough sketch of the idea behind the construction of $\Psi_{m}$.

Let us first explain (a minor variant of) the construction of $\Psi_{m}$ in dimension $d=1$, where it is rather simple. Suppose that $h$ is a 1-dimensional quasi-periodic HHF with slope $6 \cdot \ell>0$ (the case that the slope is negative is treated analogously). One can look for the minimal $w \geq 0$ such that $h(w)=2$ and for the maximal $u \leq 0$ such that 

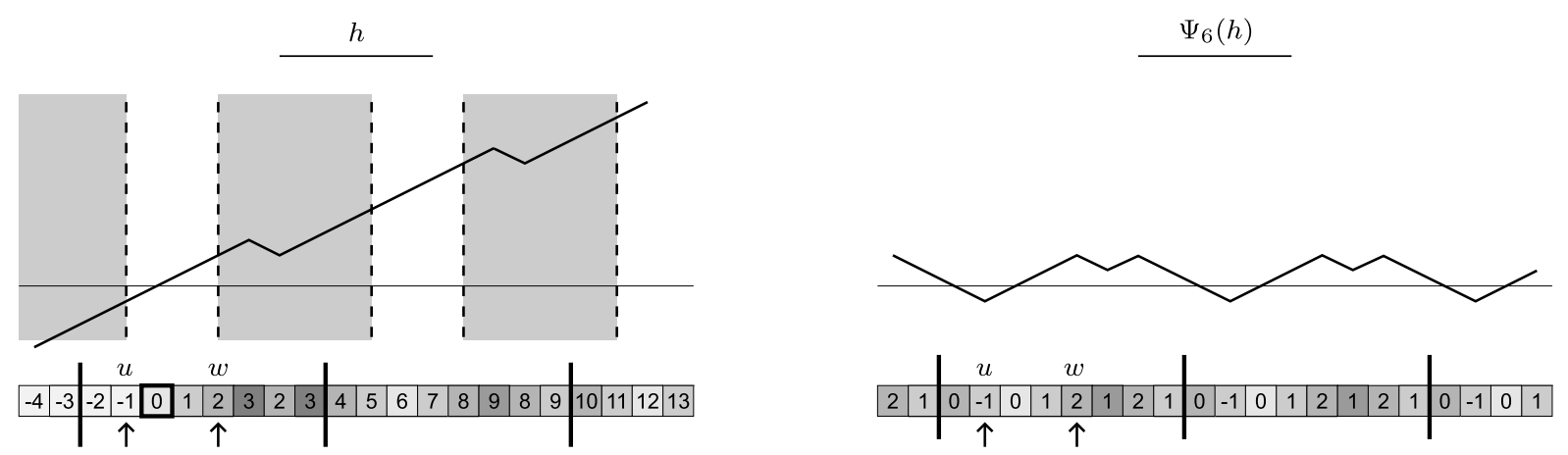

Fig. 3. On the left - an example of a one-dimensional quasi periodic HHF with $n=8$ and slope 6 . The gray regions are the regions where $\Psi_{6}$ reverses the gradient of the function. On the right - the image of the same HHF through $\Psi_{6}$.

$h(u)=-3 \ell+2$. Since $h$ has slope $6 \ell$ it follows that $w-u<n$. Thus, we may partition $\mathbb{Z}$ to segments of the form $(u+i n, w+i n]$ and $(w+i n, u+(i+1) n], i \in \mathbb{Z}$. We may then define, for $v \in \mathbb{Z}$,

$$
\Psi_{6 \ell}(h)(v)= \begin{cases}h(v)-6 i \ell, & u+i n \leq v \leq w+i n \text { for some } i \in \mathbb{Z}, \\ 4-h(v)-6 i \ell, & w+i n \leq v \leq u+(i+1) n \text { for some } i \in \mathbb{Z} .\end{cases}
$$

An example is shown in Figure 3.

It is not difficult to check that $\Psi_{6 \ell}(h)$ is still an HHF, noting that the action of $\Psi_{6 \ell}$ can be seen as reversing the gradient of $h$ between $w$ and $u+n$ and each of their translations by multiples of $n$. Moreover, the resulting HHF will be periodic in the sense that $\Psi_{6 \ell}(h)(v+n)=\Psi_{6 \ell}(h)(v)$ for all $v \in \mathbb{Z}$. To see that $\Psi_{6 \ell}$ is one-to-one, one may check that $w$ is the minimal in $\mathbb{Z}_{+}$satisfying $\Psi_{6 \ell}(h)(w)=2$ and $u$ is the maximal in $\mathbb{Z}_{-}$satisfying $\Psi_{6 \ell}(h)(u)=-3 \ell+2$. Given $\ell$, one can thereby recover $u$ and $w$ from $\Psi_{6 \ell}(h)$ and use them to recover $h$.

Generalizing this technique to higher dimensions is not immediate. The general idea is to use the given HHF $h$ to carefully define two sets $U, W \subseteq \mathbb{Z}^{d}$ and a vector $\Delta \in n \mathbb{Z}^{d}$ suitable for our purposes. The set $U$ is the analog of the interval $(-\infty, u]$ and the set $W$ is the analog of the interval $(-\infty, w]$. Among the properties which these sets satisfy is the fact that if we define $U_{i}:=U+i \Delta$ and $W_{i}:=W+i \Delta$ then the sets $\left(W_{i} \backslash U_{i}\right)$ and $\left(U_{i+1} \backslash W_{i}\right)$ form a partition of $\mathbb{Z}^{d}$. We then define $\Psi_{m}$, analogously to the above one-dimensional case, by reversing the gradient of $h$ in the regions $\left(U_{i+1} \backslash W_{i}\right)$, see (5.11). The main difficulty is to find such sets $W, U$, and vector $\Delta$, for which this operation yields a periodic $\mathrm{HHF}$, and is moreover invertible given $m$.

To show that that the size of the image of $\Psi_{m}$ is small compared to $\left|\mathrm{QP}_{\mathbf{0}}\right|$, we find an additional set $V$, sandwiched between $U$ and $W$ such that $\partial V$ is a level set of both $h$ and $\Psi_{m}(h)$. We recall that $\pi$ defines a bijection between periodic HHFs on $\mathbb{Z}^{d}$ and HHFs on $\mathbb{T}_{n}^{d}$, and show that $\pi \circ \Psi_{m}(h)$ has a level set which contains $\pi(\partial V)$ ( $\pi$ extends naturally to a mapping of the edges of $\mathbb{Z}^{d}$ to the edges of $\left.\mathbb{T}_{n}^{d}\right)$. After proving that $|\pi(\partial V)| \geq n^{d-1}$, we use a result from [18] to show that the probability that an HHF on $\mathbb{T}_{n}^{d}$ contains such a long level set is exponentially small in $n^{d-1}$. It follows that $\left|\mathrm{QP}_{m}\right| /\left|\mathrm{QP}_{\mathbf{0}}\right|$ is tiny for all $m \neq \mathbf{0}$.

The sets $U, V, W$ which we define are closely related to the level sets of the function $h$ in the sense that $h$ is constant on $\partial_{\bullet} U, \partial_{\bullet} V, \partial_{\bullet} W, \partial_{\circ} U, \partial_{\circ} V$ and $\partial_{\circ} W$. In addition, they satisfy special topological properties. The boundaries $\partial U$, $\partial V$ and $\partial W$, regarded as a collection of plaquettes in $\mathbb{R}^{d}$, are analogs of continuous hypersurfaces. Furthermore, the projection of these boundaries to the torus are analogs of hypersurfaces whose removal does not disconnect the torus.

The existence of sets $U, V, W$ satisfying all the required properties is far from obvious. The intuition for it comes from algebraic topology, specifically de Rham cohomology theory, and some of the connections are explained in the next section. However, our proof proceeds by developing the theory fully in the discrete setup. This is achieved in Sections 3 and 4. This theory is then applied in Section 5 to define $\Psi_{m}$ and prove that it satisfies the required properties.

To get a feeling of why the sets $U$ and $W$ exist, it may help to think first of continuous linear functions on $\mathbb{R}^{d}$. A multidimensional linear function is always simply a projection on its gradient vector. Such a linear function could be made periodic by periodically reversing its gradient between two hyperplanes which are perpendicular to the gradient 
vector. These hyperplanes are the analogs of $\partial W$ and $\partial U$. This case is therefore very similar to the one-dimensional case. Algebraic topology tells us that every continuous function is a deformation of a linear function. Thus, a guiding intuition may be that for more general functions, the above hyperplanes are deformed into some hypersurfaces, and hence should still exist.

\subsection{Relation with topology}

The proof of Theorem 2.3 is motivated by ideas from algebraic topology. One element of the proof that might puzzle a reader who lacks topological background is our ability to find a domain, bounded by two hypersurfaces, such that reversing the gradient in translated copies of this domain suffices to make our HHF periodic. We dedicate this short section to highlight some of the analogies between concepts of the proof and their continuous topological counterparts and shed some light on this particular point.

We begin with a brief review of concepts from de Rham cohomology theory. A 0-form on a manifold is simply a smooth function. A 1-form is a differential form which can be integrated against paths. On Riemannian manifolds a 1-form can be identified with a vector field through the Riemannian metric. A 1-form is called closed if it satisfies that its integral over contractible loops is 0 . The gradient of a 0 -form is always a closed 1 -form, and, locally, the converse is also true. Globally, however, on non-contractible manifolds such as the torus, there are many closed 1-forms which are not the gradient of any 0 -form. The group of closed 1 -forms modulo the gradients of the 0 -forms is called the first de Rham cohomology group of the manifold.

In the context of our work, 0 -forms correspond to HHFs on the torus. Closed 1-forms correspond to proper 3colorings of the torus, in the sense that, locally, they describe the discrete gradient of an HHF. In the continuous torus every closed 1 -form is locally the gradient of a 0 -form. Similarly, in the discrete torus, every 3 -coloring is locally the gradient of an HHF. However, the local information does not always add up to form the global structure of an HHF.

Algebraic topology tells us that the first de Rham cohomology measures this global obstruction, in the sense that a 1 -form corresponds to the zero class of the cohomology group if and only if it is globally the gradient of a 0 -form. The first de Rham cohomology of the $d$-dimensional torus is $\mathbb{R}^{d}$. The class of any given 1 -form can be identified by the integral of the form over a loop in each of the standard basis directions. In the terminology of this paper, this vector of integrals is called the slope of the form. Another way to represent the slope of a 1-form is to look at its pullback to what is called the universal cover of our space. In the case of the torus we look at quasi-periodic functions over $\mathbb{R}^{d}$. Taking this point of view, the slope is the vector of differences between the quasi-periodic function at standard basis points and at 0 .

Poincare duality identifies $H^{1}$, the first cohomology group of the torus, with $H_{d-1}$, the $(d-1)$ th homology group of the torus, which corresponds, if the slope consists of integers, to a class of hypersurfaces of codimension 1 . The duality further tells us that for every nice enough 1-form in a class of $H^{1}$, there exist hypersurfaces in the dual class in $H_{d-1}$, orthogonal to the gradient of the form and with the following property. Cutting the torus along such a hypersurface leaves the torus connected, but nullifies the cohomology class, i.e., on the cut torus the 1-form becomes the gradient of a 0 -form.

Much of the above description carries over to the discrete case. Here too, we match proper 3-colorings with quasiperiodic HHFs, and classify them according to their slope. We find "level sets", corresponding to the above hypersurfaces, along which one may cut the torus, that is, remove the corresponding edges, to make the coloring the gradient of an HHF. We consider two such level sets with a specific height difference. Deleting the edges of these level sets splits the torus into two connected components such that on each component, the coloring is the gradient of an HHF. Since the height of the HHF is constant along each boundary of the cut torus (as we have cut along level sets), we may reverse the gradient of the coloring on one of the connected components of the cut torus to obtain a coloring which is globally the gradient of an HHF (here, our specific choice of the height difference of the level sets enters). This illustrates the operation of $\Psi_{m}$. In practice, we transfer most of the topological part of the proof to statements involving HHFs on $\mathbb{Z}^{d}$, the universal cover of the torus. This gives us more direct access to the level sets.

The main difficulties in our task are to define the level sets in the discrete setup and to do so in such a way that would allow their recovery after applying the gradient-reversal operation. As mentioned above, the topological arguments are applicable to nice functions, with nice level sets. In the discrete setting the level sets are made out of plaquettes that can have complicated intersections, of various dimensions. Proving that discrete level sets still possess a nice structure requires the theory developed in Sections 3 and 4. 
It remains unclear whether it is possible to avoid any combinatorial argument in our proof, and use only topology. One can hope to achieve this either by defining a clever discrete variant of the de Rham cohomology, or by mapping the discrete problem to an analogous question in $\mathbb{R}^{d}$ with the hope of tackling it there. This, however, is a path we did not pursue.

\section{Closed hypersurfaces in $\mathbb{Z}^{d}$}

In this section we introduce a class of subsets of $\mathbb{Z}^{d}$ and discuss the topological properties of its members. The definitions and results are inspired by continuous topological analogs in $\mathbb{R}^{d}$ but are given directly in the discrete setting without requiring knowledge of the continuous notions (see Section 2.4 for more on the connection). We make no mention of neither colorings nor height functions here and thus the section may be read using only the definitions regarding set operations in Section 2. The tools developed here are applied to the study of colorings and height functions in the following section, but we believe that they are also of independent interest and may be of use for other purposes.

The ultimate conclusion of the discussion here, Theorem 3.4 below, is a certain trichotomy for systems of translates in $\mathbb{Z}^{d}$. This trichotomy is later applied to level sets of quasi-periodic HHFs.

We remind the reader that in the beginning of Section 2 we fixed an even integer $n$ for the remainder of the paper. This integer plays the role of the side length of the torus $\mathbb{T}_{n}^{d}$ in later sections. In this section $n$ will also play a role, though the torus $\mathbb{T}_{n}^{d}$ will not be explicitly mentioned. We point out that, unlike the rest of the paper, the results and proofs presented in this section remain valid regardless of whether $n$ is even or odd.

The structure of the section is as follows. In Section 3.1 we present the fundamental properties of the sets that we investigate and state our two main results, in the form of certain trichotomies. Section 3.2 describes corollaries of the main results, which will be of use in our application. The proofs of the main results are given in Sections 3.3 and 3.4.

\subsection{Topology of $\mathbb{Z}^{d}$}

We begin by defining three properties of sets in $\mathbb{Z}^{d}$ : co-connectedness, boundary disjointness, and translation respecting. These are repeatedly used throughout the paper.

\section{Co-connectedness}

A set $U \subseteq \mathbb{Z}^{d}$ is called co-connected if $U \neq \varnothing, U \neq \mathbb{Z}^{d}$ and $U$ and $U^{c}$ are connected.

A useful property of co-connected sets is that their boundaries are, in a sense, connected. Namely,

Proposition 3.1. If $A$ is a co-connected set in $\mathbb{Z}^{d}$ then $\partial_{\bullet} A \cup \partial_{\circ} A, A^{++} \backslash A$ and $A \backslash A^{--}$are all connected sets.

We delay the proof of this proposition to Section 3.3, as it requires the tools developed there.

In order to get a more intuitive grasp of the theorems and definitions of this section the reader might find it useful to regard $\mathbb{Z}^{d}$ as a lattice of $d$-dimensional cubes where the edges between adjacent vertices represent plaquettes of codimension 1. Taking this continuous view, co-connected sets are analogous to continuous sets whose boundary is a connected, oriented, closed hypersurface. A set and its complement should be thought of as defining opposite orientations on the same surface.

\section{Boundary disjointness}

Two sets $U_{1}, U_{2} \subseteq \mathbb{Z}^{d}$ are called boundary disjoint if

1. $\partial U_{1} \cap \partial U_{2}=\varnothing$,

2. there is no 4-cycle in $\mathbb{Z}^{d}$ whose vertices, in order, are $\left(v_{00}, v_{01}, v_{11}, v_{10}\right)$ such that $v_{00} \in U_{1}^{c} \cap U_{2}^{c}, v_{01} \in U_{1}^{c} \cap U_{2}$, $v_{11} \in U_{1} \cap U_{2}$ and $v_{10} \in U_{1} \cap U_{2}^{c}$.

Here and below, by a cycle in $\mathbb{Z}^{d}$ we mean a finite set $\left\{\left(u_{1}, v_{1}\right), \ldots,\left(u_{k}, v_{k}\right)\right\}$ of distinct edges of $\mathbb{Z}^{d}$ satisfying that $u_{i+1}=v_{i}, 1 \leq i \leq k-1$, and $u_{1}=v_{k}$. A 4-cycle is a cycle with $k=4$, and by its vertices, in order, we mean $\left(u_{1}, u_{2}, u_{3}, u_{4}\right)$. 
Continuing the analogy with hypersurfaces, two sets are boundary disjoint if their boundaries neither overlap nor intersect transversally.

When both $U_{1}$ and $U_{2}$ are odd, as will always be the case from Section 4 and on, the second condition for boundary disjointness is trivially fulfilled, yielding the simpler relation:

odd $U_{1}, U_{2}$ are boundary disjoint iff $\partial U_{1} \cap \partial U_{2}=\varnothing$.

Observe that, by definition, boundary disjointness is preserved under taking complements, i.e., if $U_{1}, U_{2}$ are boundary disjoint sets, then each of the pairs $\left\{U_{1}^{c}, U_{2}\right\},\left\{U_{1}, U_{2}^{c}\right\}$ and $\left\{U_{1}^{c}, U_{2}^{c}\right\}$ are also boundary disjoint.

The containment relations between two co-connected boundary disjoint sets are restricted by the following theorem.

Theorem 3.2 (Pair trichotomy). If $U_{1}, U_{2} \subseteq \mathbb{Z}^{d}$ are co-connected and boundary disjoint sets, then exactly one of the following alternatives holds:

- $U_{1} \cap U_{2}=\varnothing$,

- $U_{1}^{c} \cap U_{2}^{c}=\varnothing$,

- $U_{1} \subsetneq U_{2}$ or $U_{2} \subsetneq U_{1}$.

The proof of this theorem is postponed to Section 3.3.

The following proposition relates containment of boundary disjoint sets and their distance from a third set.

Proposition 3.3. If $U_{1}, U_{2} \subseteq \mathbb{Z}^{d}$ are non-empty, boundary disjoint sets satisfying $U_{1} \subset U_{2}$ then for every non-empty set $V$ satisfying $V \cap U_{2}=\varnothing$ we have $\operatorname{dist}\left(U_{1}, V\right)>\operatorname{dist}\left(U_{2}, V\right)$.

Proof. Using boundary disjointness and (2.1), we have $U_{1}^{+} \subseteq U_{2}$. By (2.2) and (2.3) we thus have $\operatorname{dist}\left(U_{1}, V\right)>$ $\operatorname{dist}\left(U_{2}, V\right)$ as required.

Translation respecting sets

For a set $U \subseteq \mathbb{Z}^{d}$, we define $T_{U}=T_{U}^{n}$, the set of translates of $U$ by multiples of $n$ in each of the coordinate directions, as

$$
T_{U}:=\left\{U+x: x \in n \mathbb{Z}^{d}\right\},
$$

recalling that $U+v:=\{u+v: u \in U\}$. We note that it may well be the case that different translations of $U$ yield the same set.

A set $U \subseteq \mathbb{Z}^{d}$ is called translation respecting if $U$ is co-connected and every distinct $U_{1}, U_{2} \in T_{U}$ are boundary disjoint. Observe that, by definition, if $U$ is translation respecting, then so is $U^{c}$.

Continuing the analogy with hypersurfaces, a translation respecting set is analogous to a hypersurface in $\mathbb{R}^{d}$, which satisfies that the projection of $\mathbb{R}^{d}$ to the continuous torus maps its boundary to a closed hypersurface.

The main result of this section is that the trichotomy of Theorem 3.2 extends to translation respecting sets in the following strong sense.

Theorem 3.4 (Translation trichotomy). If $U \subseteq \mathbb{Z}^{d}$ is translation respecting and $\left|T_{U}\right|>1$, then exactly one of the following alternatives holds:

- [Type 1] If $U_{1}, U_{2} \in T_{U}$ and $U_{1} \neq U_{2}$ then $U_{1} \cap U_{2}=\varnothing$.

- [Type -1] If $U_{1}, U_{2} \in T_{U}$ and $U_{1} \neq U_{2}$ then $U_{1}^{c} \cap U_{2}^{c}=\varnothing$.

- [Type 0] If $U_{1}, U_{2} \in T_{U}$ then $U_{1} \subseteq U_{2}$ or $U_{2} \subseteq U_{1}$.

Moreover, if $U$ satisfies the Type 0 alternative of the theorem, then there exists a unique order-preserving bijection $o: T_{U} \rightarrow \mathbb{Z}$ such that $o(U)=0$. Here, order preserving means that $o\left(U_{1}\right)<o\left(U_{2}\right)$ if and only if $U_{1} \subsetneq U_{2}$. Furthermore, there exists a $\Delta \in n \mathbb{Z}^{d}$ such that $o^{-1}(i+1)=o^{-1}(i)+\Delta$ for all $i \in \mathbb{Z}$. We call any such $\Delta$ a minimal translation of $U$. 

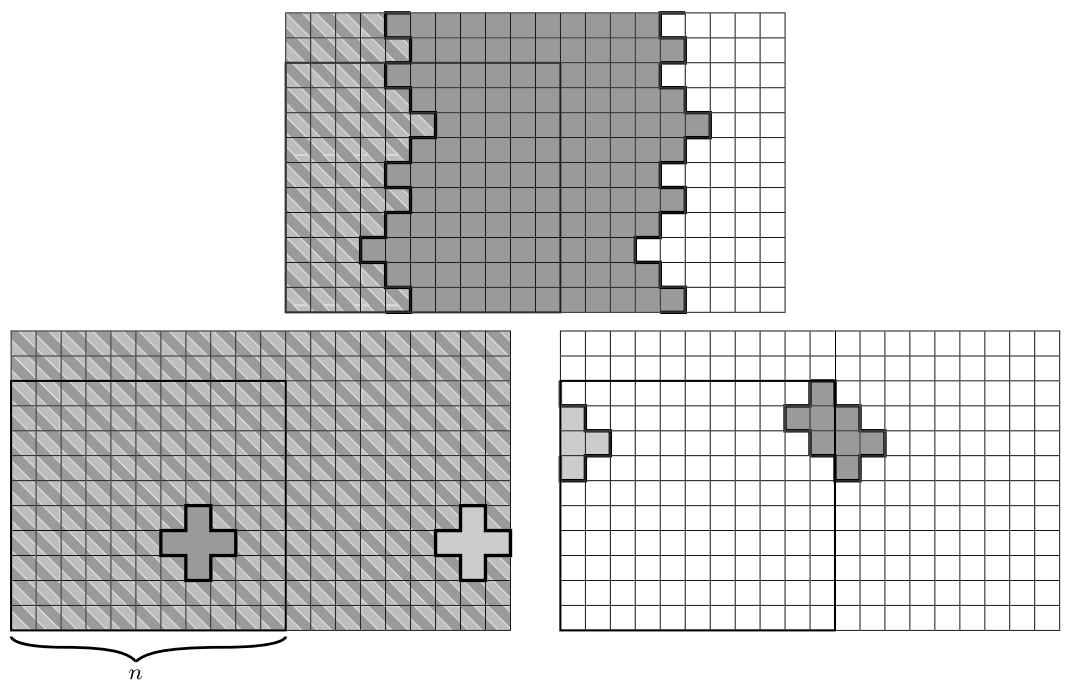

Fig. 4. Examples of translation respecting sets of the three types. In each image a portion of the plane is depicted, on which a set $U$ and its translation $U+n e_{1}$ are emphasized in light gray and in dark gray respectively. Vertices contained in both sets are striped. In each image a different alternative of Theorem 3.4 holds: At the top type 0 , at the bottom-left type -1 and at the bottom-right type 1.

The proof of this theorem is postponed to Section 3.4.

We remark regarding the assumption that $\left|T_{U}\right|>1$ that while in dimension $d=2$ any co-connected set $U$ has $\left|T_{U}\right|>1$ (recalling that a co-connected set is assumed to be different from $\varnothing$ and $\mathbb{Z}^{d}$ ), there do exist co-connected sets $U$ in dimensions $d \geq 3$ having $\left|T_{U}\right|=1$ (for instance, the set of vertices in $\mathbb{Z}^{d}$ having at most one coordinate which is not a multiple of $n$ ).

Theorem 3.4 allows us to assign a type to every translation respecting set $U$ satisfying $\left|T_{U}\right|>1$. For $i \in\{-1,0,1\}$, we write $\operatorname{Type}(U)=i$ if $U$ satisfies the Type $i$ alternative of the theorem. The case $\left|T_{U}\right|=1$ has little bearing on our application. However, for completeness, we say in this case, with a slight abuse of notation, that both Type $(U)=1$, $\operatorname{Type}(U)=-1$ and Type $(U) \neq 0$ hold. An illustration of sets of the various types is given in Figure 4.

\subsection{Corollaries of the trichotomy}

In this section we state several useful corollaries of Theorem 3.4. The next proposition discusses how the type of translation respecting sets is affected by taking complements.

Proposition 3.5. If $U$ is translation respecting of type $i$ then:

- $U^{c}$ is translation respecting of type $-i$.

- If $U$ is of type 0 with minimal translation $\Delta$, then $-\Delta$ is a minimal translation of $U^{c}$.

The proof of this proposition is straightforward from Theorem 3.4.

The following proposition investigates the possible containment relations between translation respecting sets.

Proposition 3.6. Let $U, V$ be two translation respecting sets satisfying that $\left|T_{U}\right|,\left|T_{V}\right|>1$ and $U \subseteq V$. Then Type $(U) \geq \operatorname{Type}(V)$.

Proof. Our goal is to show that (Type $(U)$, Type $(V)) \notin\{(-1,0),(-1,1),(0,1)\}$. Equivalently, we need to show that

if $\operatorname{Type}(V)=1$ then $\operatorname{Type}(U)=1$,

if $\operatorname{Type}(U)=-1$ then $\operatorname{Type}(V)=-1$. 
Suppose first that Type $(V)=1$. Let $\Delta \in n \mathbb{Z}^{d}$ be such that $V+\Delta \neq V$ (which exists as $\left|T_{V}\right|>1$ ). As $\operatorname{Type}(V)=1$, $V \cap(V+\Delta)=\varnothing$. Thus, as $U \subseteq V$ and $U+\Delta \subseteq V+\Delta$ we deduce that

$$
U \cap(U+\Delta)=\varnothing \text {. }
$$

In particular, $U \neq U+\Delta$ whence $U$ and $U+\Delta$ are boundary disjoint (as $U$ is translation respecting) and the pair trichotomy, Theorem 3.2, implies that

$$
U^{c} \cap(U+\Delta)^{c} \neq \varnothing .
$$

The translation trichotomy, Theorem 3.4, and the relations (3.4) and (3.5) imply that Type $(U)=1$, establishing (3.2)

Now observe that $U^{c}, V^{c}$ are also translation respecting and satisfy $V^{c} \subseteq U^{c}$. Thus, we may apply (3.2) with $(U, V)$ replaced by $\left(V^{c}, U^{c}\right)$ and deduce from Proposition 3.5 that (3.3) holds.

Translation respecting sets of type 0

These have a unique structure, as the following proposition indicates.

Proposition 3.7. If $U$ is translation respecting of type 0 then:

- $\bigcup_{V \in T_{U}} V=\mathbb{Z}^{d}$.

- There exists $1 \leq i \leq d$ such that for every $v \in \mathbb{Z}^{d},\left\{v+k e_{i}: k \in \mathbb{Z}\right\}$ intersects both $U$ and $U^{c}$.

- If $U+n e_{1} \neq U$ then for every $v \in \mathbb{Z}^{d},\left\{v+k e_{1}: k \in \mathbb{Z}\right\}$ intersects both $U$ and $U^{c}$.

Proof. Let $v \in \mathbb{Z}^{d}$ and let $\Delta$ be a minimal translation of $U$. Observe that by definition, $U \subsetneq U+\Delta$, and $U, U+\Delta$ are co-connected and boundary disjoint. Applying Proposition 3.3 we get $\operatorname{dist}(U+\Delta,\{v\}) \leq \max (\operatorname{dist}(U,\{v\})-1,0)$. Iterating, we obtain that there exists some $k$ such that $v \in U+k \Delta$. We deduce the first item of the proposition.

The second item follows from the third by symmetry, as the fact that $U+\Delta \neq U$ (using that $U$ is of type 0 ) implies that there exists some $1 \leq i \leq d$ for which $U+n e_{i} \neq U$. We proceed to prove the third item. Observe that by the last part of Theorem 3.4, there exists some $\ell \in \mathbb{Z} \backslash\{0\}$ such that $U+n e_{1}=U+\ell \Delta$. It follows also that $U^{c}+n e_{1}=U^{c}+\ell \Delta$. Notice that both $U$ and $U^{c}$ are translation respecting of type 0 with $-\Delta$ being a minimal translation for $U^{c}$ (by Proposition 3.5). Thus, the first item of the proposition and the last part of Theorem 3.4 show that for every $v \in \mathbb{Z}^{d}$ there exist $k_{1}, k_{2} \in \mathbb{Z}$ such that

$$
v \in\left(U+k_{1} \ell \Delta\right) \cap\left(U^{c}+k_{2} \ell \Delta\right) .
$$

Equivalently $v-k_{1} n e_{1} \in U$ while $v-k_{2} n e_{1} \notin U$, as required.

Recall that $\pi$ is the projection of $\mathbb{Z}^{d}$ onto $\mathbb{T}_{n}^{d}$. It naturally extends to a mapping of the edges of $\mathbb{Z}^{d}$ to the edges of $\mathbb{T}_{n}^{d}$. The projection of the boundary of translation respecting sets of type 0 through $\pi$ is very long, as the following lemma shows.

Lemma 3.8. If $V$ is a translation respecting set of type 0 with minimal translation $\Delta$ satisfying $V+\ell \Delta=V+n e_{1}$ then

$$
\left|\left\{\left(w_{0}, w_{1}\right) \in \pi(\partial V): w_{0}-w_{1}=e_{1}\right\}\right| \geq \ell n^{d-1} .
$$

Proof. The lemma holds trivially if $\ell=0$. Assume without loss of generality that $\ell>0$. We write

$$
X:=\left\{x \in \mathbb{Z}^{d}: \forall j \in\{2, \ldots, d\} 0 \leq x_{j}<n\right\} .
$$

Observe that $\pi(\partial V)=\pi(\partial V+k \Delta)$ for all $k \in \mathbb{Z}$. Thus, to obtain the lemma it would suffice to show the following two claims:

$$
\left\{\pi\left(X \cap\left(\partial_{\bullet} V+k \Delta\right)\right)\right\}_{k \in\{0, \ldots, \ell-1\}} \text { are disjoint. }
$$

For each $k \in\{0, \ldots, \ell-1\}$ we have $\left|\pi\left(E_{1}(X) \cap(\partial V+k \Delta)\right)\right| \geq n^{d-1}$,

where $E_{1}(X):=\left\{\left(x, x+e_{1}\right): x \in X\right\}$. 
We begin by showing (3.6). Since $V$ is translation respecting, $\left\{X \cap\left(\partial_{\bullet} V+k \Delta\right)\right\}_{k \in\{0, \ldots, \ell-1\}}$ are disjoint. Thus, to obtain (3.6), all that remains is to show that for all pairs of distinct $k_{1}, k_{2} \in\{0, \ldots, \ell-1\}$, there are no two elements $x_{1} \in \partial_{\bullet} V+k_{1} \Delta, x_{2} \in \partial_{\bullet} V+k_{2} \Delta$, such that $x_{1}+a n e_{1}=x_{2}$ for some $a \in \mathbb{N}$. Indeed, in such a case, we would have $x_{2}=x_{1}+a_{n} e_{1} \in \partial_{\bullet} V+k_{1} \Delta+a n e_{1}=\partial_{\bullet} V+\left(k_{1}+a \ell\right) \Delta$, which would imply, by boundary disjointness, that $V+k_{2} \Delta=V+\left(k_{1}+a \ell\right) \Delta$, and hence $k_{2}=k_{1}+a \ell$ which contradicts our assumption. (3.6) follows.

To see (3.7), observe that by the third item of Proposition 3.7, for every $x \in \mathbb{Z}^{d}, k \in\{0,1, \ldots, \ell-1\}$, there exists a $q \in \mathbb{Z}$ such that

$$
\left(x+q e_{1}, x+(q+1) e_{1}\right) \in \partial V+k \Delta .
$$

Using (3.8) for all $x \in X$ satisfying that $x_{1}=0$, we obtain (3.7).

\subsection{Proof of the pair trichotomy}

In this section we prove Proposition 3.1 and Theorem 3.2 using the approach of Timár in [22]. To do so, we make use of the well-known fact that 4-cycles span the cycles of $\mathbb{Z}^{d}$, i.e., every cycle $\sigma$ in $\mathbb{Z}^{d}$ can be written as

$$
\sigma=\sum_{c \in \mathcal{C}} c
$$

where $\mathcal{C}$ is a set of 4-cycles, and we interpret the sum as meaning that an edge is in $\sigma$ if it appears in an odd number of cycles in $\mathcal{C}$.

To aid our proof we introduce the following family of graphs.

Definition 3.9. Given $U \subseteq \mathbb{Z}^{d}$, a set of vertices, we define a graph $G_{U}$ as follows. The vertices of $G_{U}$ are the vertices of $\mathbb{Z}^{d}$. Two vertices $u, v$ are adjacent in $G_{U}$ if there exist $e_{u}, e_{v} \in \partial U$ and a 4-cycle $c$, such that $u \in e_{u}, v \in e_{v}$, and $e_{u}, e_{v} \in c$.

The following lemma connects this definition with co-connected sets.

Lemma 3.10. If $U \subset \mathbb{Z}^{d}$ is a co-connected set of vertices, then $\partial_{\bullet} U$ is connected in $G_{U}$.

Proof. The proof is heavily based on ideas developed in [22]. It suffices to show that for any non-trivial partition $S_{1}, S_{2}$ of $\partial_{\bullet} U$ there exists an edge of $G_{U}$ connecting $S_{1}$ and $S_{2}$. Here, a non-trivial partition means that $S_{1}, S_{2} \neq \varnothing$, $S_{1} \cap S_{2}=\varnothing$ and $S_{1} \cup S_{2}=\partial_{\bullet} U$. Let $S_{1}, S_{2}$ be such a partition. We set

$$
\begin{aligned}
& E_{1}:=\left\{e \in \partial U: e \cap S_{1} \neq \varnothing\right\}, \\
& E_{2}:=\left\{e \in \partial U: e \cap S_{2} \neq \varnothing\right\} .
\end{aligned}
$$

By the connectedness of $U$ and $U^{c}$ in $\mathbb{Z}^{d}$, there exists some cycle $\sigma$ in $\mathbb{Z}^{d}$ which contains exactly one edge of $E_{1}$ and one edge of $E_{2}$ (in fact, we can even pick those boundary edges arbitrarily). As 4-cycles span the cycles of $\mathbb{Z}^{d}$, we write $\sigma$ as a sum of such cycles

$$
\sigma=\sum_{c \in \mathcal{C}} c
$$

as in (3.9). We notice that as $\sigma$ contains an odd number of $E_{1}$ edges (in fact, just one), there must also be a 4-cycle $c_{0} \in \mathcal{C}$ containing an odd number of $E_{1}$ edges. However as every cycle contains an even number of edges from the boundary $\partial U=E_{1} \uplus E_{2}, c_{0}$ must contain an edge of $E_{2}$ as well. Thus $S_{1}$ and $S_{2}$ are connected by an edge of $G_{U}$, concluding the proof.

Lemma 3.10 allows us to prove Proposition 3.1 and Theorem 3.2. In this proof we will make use of [22, Theorem 4]. For convenience, we state a special case of this theorem in the context of our work. 

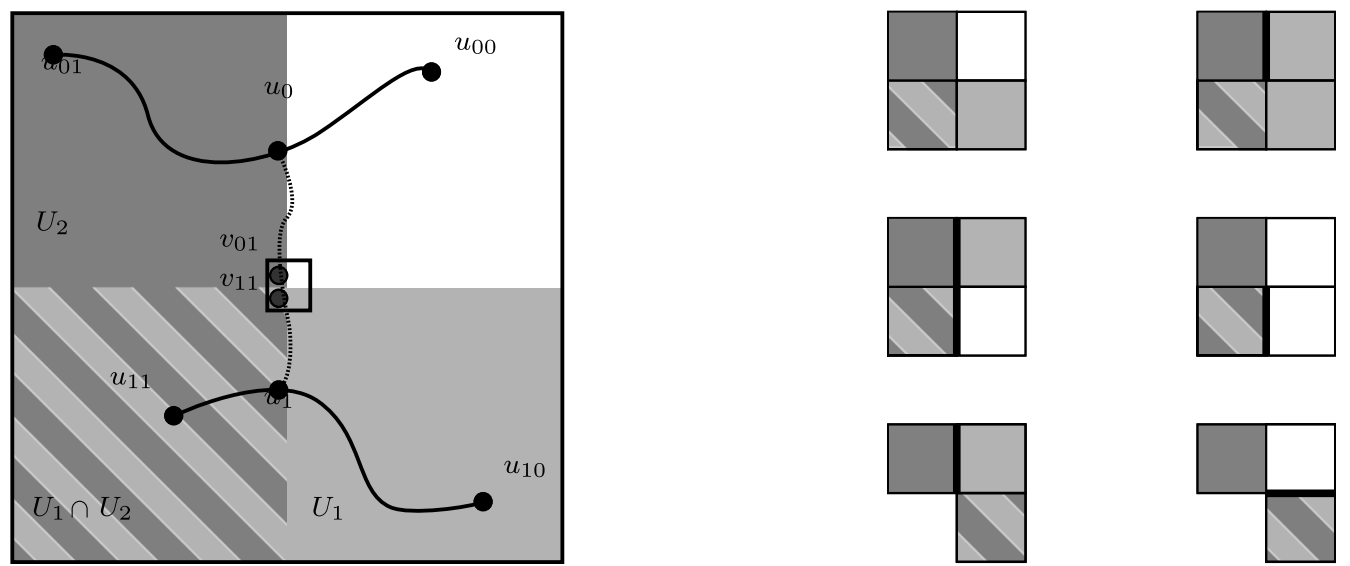

Fig. 5. Illustration accompanying the proof of Theorem 3.2. On the left - the roles of $u_{00}, u_{10}, u_{11}$ and $u_{01}$ are illustrated, as well as those of $u_{0}$, $u_{1}, v_{01}$ and $v_{11}$. On the right - all the possible configurations of the 4-cycle $c$, up to rotation and reflection, are illustrated. Observe that $c$ must contain a dark vertex $v_{01}$ and a striped vertex $v_{11}$ and that each of these must be adjacent to a vertex in $c$ which is neither dark nor striped. In the top four configurations $v_{01}$ and $v_{11}$ are next to each other while in the bottom two they are in opposite corners of the cycle. When the boundary disjointness is ruled out due to the existence of an edge violating $\partial U_{1} \cap \partial U_{2}=\varnothing$, this edge is marked. When no edge is marked, the alternative is ruled out due to the existence of a "forbidden cycle" (as in the definition of boundary disjointness).

Theorem (Timár). For any co-connected $A \subsetneq \mathbb{Z}^{d}$, the set

$$
\left\{y \in A^{c}: y \text { differs from some point in } A \text { by } \pm 1 \text { in each of exactly one or two coordinates }\right\}
$$

is connected in $\mathbb{Z}^{d}$.

To see that this is a special case of [22, Theorem 4], take $G=\mathbb{Z}^{d}$, and let $G^{+}$be $G$ with an edge between every two vertices who differ by \pm 1 on each of exactly one or two coordinates. Also, take $C=A$, and let $x$ be some arbitrary point in $A^{c}$.

Proof of Proposition 3.1. Let $A$ be a co-connected set in $\mathbb{Z}^{d}$. The first part of the proposition is an immediate result of Lemma 3.10, as connectivity of $\partial_{\bullet} A \cup \partial_{\circ} A$ in $\mathbb{Z}^{d}$ is weaker than connectivity of $\partial_{\bullet} A$ in $G_{A}$. The proof of the second part uses the above stated version of [22, Theorem 4]. By the theorem,

$$
B:=\left\{y \in A^{c}: y \text { differs from some point in } A \text { by } \pm 1 \text { in each of exactly one or two coordinates }\right\}
$$

is connected in $\mathbb{Z}^{d}$. In addition $B$ satisfies that $B \subset A^{++} \backslash A$ and that every vertex in $A^{++} \backslash A$ has a neighbor in $B$ (as $\left.A^{+} \backslash A \subset B\right)$. We therefore have that $A^{++} \backslash A$ is connected in $\mathbb{Z}^{d}$ as required. To get the third part of the proposition, we recall that if $A$ is co-connected, then so is $A^{c}$, and that $A \backslash A^{--}=\left(A^{c}\right)^{++} \backslash A^{c}$. We can therefore derive the third part of the proposition by applying the second part to $A^{c}$.

Proof of Theorem 3.2. We accompany the proof with Figure 5. Assume to the contrary all the alternatives in the theorem do not hold. We can therefore pick $u_{11} \in U_{1} \cap U_{2}, u_{10} \in U_{1} \cap U_{2}^{c}, u_{01} \in U_{1}^{c} \cap U_{2}$ and $u_{00} \in U_{1}^{c} \cap U_{2}^{c}$. As $U_{1}$ is connected, there exists a path inside $U_{1}$ between $u_{10}$ and $u_{11}$. This path must contain a vertex $u_{1} \in U_{1} \cap \partial_{\bullet} U_{2}$. Similarly there exists a path outside $U_{1}$ between $u_{00}$ and $u_{01}$ which contains a vertex $u_{0} \in U_{1}^{c} \cap \partial_{\bullet} U_{2}$.

By Lemma 3.10, $\partial_{\bullet} U_{2}$ is connected in $G_{U_{2}}$. In particular, if we partition $\partial_{\bullet} U_{2}$ into $U_{1} \cap \partial_{\bullet} U_{2}$ and $U_{1}^{c} \cap \partial_{\bullet} U_{2}$, we must have an edge in $G_{U_{2}}$ crossing this partition. In other words, there exists a 4-cycle $c$ which contains two edges $e_{0}, e_{1} \in \partial U_{2}$, and two vertices $v_{01} \in e_{0}$ and $v_{11} \in e_{1}$ such that $v_{01} \in U_{1}^{c} \cap \partial_{\bullet} U_{2}$ and $v_{11} \in U_{1} \cap \partial_{\bullet} U_{2}$. A careful case study of all the possible configurations of such a cycle (see Figure 5) yields that its existence must contradict the boundary disjointness for $U_{1}$ and $U_{2}$. We conclude that at least one of the alternatives in the theorem must hold. 
Next we show that exactly one of the alternatives holds. The third alternative cannot co-exist with either of the first two alternatives as a co-connected set is non-empty and has non-empty complement. For the first two alternatives to hold together it must be the case that $U_{1}=U_{2}^{c}$, contradicting the boundary disjointness of $U_{1}$ and $U_{2}$. The theorem follows.

\subsection{Proof of the translation trichotomy}

This section is dedicated to the proof of Theorem 3.4.

We begin by showing the trichotomy itself. The pair intersection trichotomy, Theorem 3.2, guarantees that every two sets $U_{1}, U_{2} \in T_{U}$ satisfy one of the three alternatives of the theorem. Thus it is sufficient to show that for any three distinct sets $U_{1}, U_{2}, U_{3} \in T_{U}$, the same alternative holds for both pairs $U_{1}, U_{2}$ and $U_{1}, U_{3}$. In particular, the theorem is immediate if $\left|T_{U}\right|=2$. Fix distinct $U_{1}, U_{2}, U_{3} \in T_{U}$. We shall rule out three cases.

1. Alternatives 0 and 1 cannot coexist. Let $\delta, \Delta \in n \mathbb{Z}^{d}$ be such that $U_{2}=U_{1}+\delta$ and $U_{3}=U_{1}+\Delta$. Assume, WLOG, that $U_{1} \cap U_{3}=\varnothing$ and $U_{1} \subsetneq U_{2}$. As $U_{1}$ and $U_{2}$ are boundary disjoint, by Proposition 3.3 we get that $\operatorname{dist}\left(U_{1}, U_{3}\right)>$ $\operatorname{dist}\left(U_{2}, U_{3}\right)$. We note that, $U_{1}+\Delta \subseteq U_{1}+\Delta+\delta$, as $U_{1} \subseteq U_{1}+\delta$. We deduce, using (2.3), that dist $\left(U_{1}+\delta, U_{1}+\right.$ $\Delta) \geq \operatorname{dist}\left(U_{1}+\delta, U_{1}+\Delta+\delta\right)$. Putting all of this together, we get:

$$
\operatorname{dist}\left(U_{1}, U_{1}+\Delta\right)>\operatorname{dist}\left(U_{1}+\delta, U_{1}+\Delta\right) \geq \operatorname{dist}\left(U_{1}+\delta, U_{1}+\Delta+\delta\right)=\operatorname{dist}\left(U_{1}, U_{1}+\Delta\right),
$$

which is a contradiction.

2. Alternatives 0 and -1 cannot coexist. The argument follows similarly to the previous part by passing from $U_{1}, U_{2}, U_{3}$ to $U_{1}^{c}, U_{2}^{c}, U_{3}^{c}$.

3. Alternatives 1 and -1 cannot coexist. To see this, assume, WLOG, that $U_{1} \cap U_{2}=\varnothing$ and $U_{1}^{c} \cap U_{3}^{c}=\varnothing$. It follows that $U_{1} \cup U_{3}=\mathbb{Z}^{d}$ and hence $U_{2} \subseteq U_{3}$. A contradiction follows since alternatives 0 and 1 cannot coexist.

Next, we show the second part of the theorem, i.e., that if $\operatorname{Type}(U)=0$, then there exists a translation $\Delta \in n \mathbb{Z}^{d}$ and an order-preserving bijection $o: T_{U} \rightarrow \mathbb{Z}$, such that $o^{-1}(i+1)=o^{-1}(i)+\Delta$ for all $i \in \mathbb{Z}$. Assume Type $(U)=0$. Define $o(U):=0$ and for any $V \in T_{U}$ let

$$
o(V):= \begin{cases}\left|\left\{W \in T_{U}: U \subsetneq W \subseteq V\right\}\right|, & U \subseteq V, \\ -\left|\left\{W \in T_{U}: V \subseteq W \subsetneq U\right\}\right|, & V \subseteq U .\end{cases}
$$

To see that this is well defined, let us explain why $\left\{W \in T_{U}: U \subsetneq W \subseteq V\right\}$ is finite. A similar argument will show that $\left\{W \in T_{U}: V \subseteq W \subsetneq U\right\}$ is finite. Since $T_{U}$ is ordered by inclusion, applying Proposition 3.3 to the complements of two distinct sets in $\left\{W \in T_{U}: U \subsetneq W \subseteq V\right\}$, taking the $V$ of the proposition to be our $U$, shows that each set $W$ in $\left\{W \in T_{U}: V \subseteq W \subsetneq U\right\}$ is uniquely characterized by $\operatorname{dist}\left(W^{c}, U\right)$. Since $\operatorname{dist}\left(W^{c}, U\right) \leq \operatorname{dist}\left(V^{c}, U\right)$ we conclude that $\left\{W \in T_{U}: U \subsetneq W \subseteq V\right\}$ is finite, as we wanted to show.

To show that $o$ is one-to-one, suppose $V_{1}, V_{2} \in T_{U}$ satisfy $o\left(V_{1}\right)=o\left(V_{2}\right)$. Assume WLOG that $o\left(V_{1}\right) \geq 0$ and $V_{1} \subseteq V_{2}$. This implies that

$$
\left\{W \in T_{U}: U \subsetneq W \subseteq V_{1}\right\} \subseteq\left\{W \in T_{U}: U \subsetneq W \subseteq V_{2}\right\} .
$$

However, as $o\left(V_{1}\right)=o\left(V_{2}\right)$, we get

$$
\left\{W \in T_{U}: U \subsetneq W \subseteq V_{1}\right\}=\left\{W \in T_{U}: U \subsetneq W \subseteq V_{2}\right\}
$$

and, in particular, $V_{2} \subseteq V_{1}$. Thus $V_{1}=V_{2}$.

Finally, we show that there is a $\Delta \in n \mathbb{Z}^{d}$ such that $o^{-1}(i+1)=o^{-1}(i)+\Delta$ for all $i \in \mathbb{Z}$. We begin by observing that $o^{-1}(1)$ is nonempty. To see this recall that $\left|T_{U}\right|>1$ and therefore $U \subsetneq U+z$ for some $z \in n \mathbb{Z}^{d}$. This implies that $o(U+z) \geq 1$ and therefore there must exist some $\Delta \in n \mathbb{Z}^{d}$ such that $o(U+\Delta)=1$. Equivalently, there is no $W \in T_{U}$ for which $U \subsetneq W \subsetneq U+\Delta$. Since this situation is preserved under translations it follows that $o^{-1}(i)=U+i \Delta$ for all $i \in \mathbb{Z}$. 


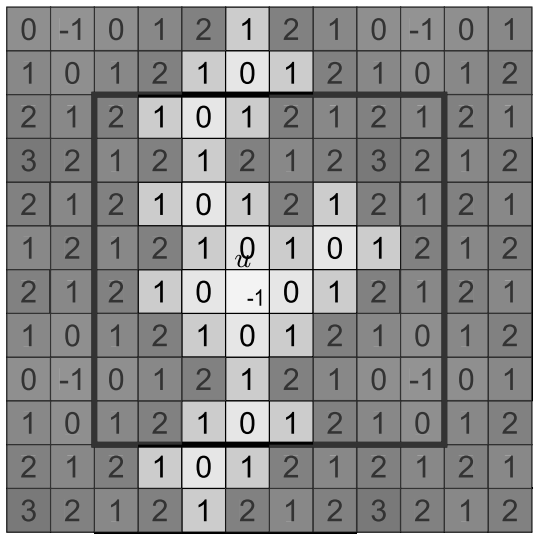

\begin{tabular}{|c|c|c|c|c|c|c|c|c|c|c|c|}
\hline 0 & -1 & 0 & 1 & 2 & 1 & 2 & 1 & 0 & -1 & 0 & 1 \\
\hline 1 & 0 & 1 & 2 & 1 & 0 & 1 & 2 & 1 & 0 & 1 & 2 \\
\hline 2 & 1 & 2 & 1 & 0 & 1 & 2 & 1 & 2 & 1 & 2 & 1 \\
\hline 3 & 2 & 1 & 2 & 1 & 2 & 1 & 2 & 3 & 2 & 1 & 2 \\
\hline 2 & 1 & 2 & 1 & 0 & 1 & 2 & 1 & 2 & 1 & 2 & 1 \\
\hline 1 & 2 & 1 & 2 & 1 & 0 & 1 & 0 & 1 & 2 & 1 & 2 \\
\hline 2 & 1 & 2 & 1 & 0 & -1 & 0 & $v$ & 2 & 1 & 2 & 1 \\
\hline 1 & 0 & 1 & 2 & 1 & 0 & 1 & 2 & 1 & 0 & 1 & 2 \\
\hline 0 & -1 & 0 & 1 & 2 & 1 & 2 & 1 & 0 & -1 & 0 & 1 \\
\hline 1 & 0 & 1 & 2 & 1 & 0 & 1 & 2 & 1 & 0 & 1 & 2 \\
\hline 2 & 1 & 2 & 1 & 0 & 1 & 2 & 1 & 2 & 1 & 2 & 1 \\
\hline 3 & 2 & 1 & 2 & 1 & 2 & 1 & 2 & 3 & 2 & 1 & 2 \\
\hline
\end{tabular}

Fig. 6. An illustration of sublevel components for a certain periodic $h \in \operatorname{Hom}\left(\mathbb{Z}^{d}\right)$, with respect to the two vertices $u$, $v \in \mathbb{Z}^{d}$. On the left - a portion of $\mathrm{LC}_{h}^{1+}(u)$ is highlighted. On the right - a portion of $\operatorname{LC}_{h}^{1+}(u, v)$. Observe that $\mathrm{LC}_{h}^{1+}(u, v)$ is co-connected while $\mathrm{LC}_{h}^{1+}(u)$ is not.

\section{Sublevel sets of HHFs}

In this section we establish the theoretical basis for dealing with quasi-periodic HHFs. Much of the intuition behind the theorems of this section stems from algebraic topology, viewing quasi-periodic HHFs as a discrete analogue of co-cycles on the torus, and periodic HHFs as a discrete analogue of co-boundaries. Nonetheless, we avoid making any direct reference to topology, and restrict ourselves to purely combinatorial proofs. The results of this section are central to our construction in Section 5 of the one-to-one mapping $\Psi_{m}: \mathrm{QP}_{m} \rightarrow \mathrm{QP}_{\mathbf{0}}$ and the analysis of its properties.

We begin by introducing the notions of sublevel sets and sublevel components of HHFs. Sublevel sets are discrete counterparts to sublevel sets of continuous functions. A sublevel component augments a sublevel set to a co-connected set.

Let $G$ be either $\mathbb{Z}^{d}$ or $\mathbb{T}_{n}^{d}$. Let $k \in \mathbb{Z}, h \in \operatorname{Hom}(G)$ and let $u, v \in V(G)$ satisfy

$$
h(u) \leq k<h(v) .
$$

We define the $k$-sublevel set of $u$,

$$
\operatorname{LC}_{h}^{k+}(u) \text { is the connected component of } u \text { in } G \backslash\{w \in V(G): h(w)=k+1\} .
$$

While the sublevel set is itself connected, by definition, its complement may be disconnected. We wish to isolate a single connected component of the complement and do this by enlarging the sublevel set. Precisely, we define the $k$-sublevel component from $u$ to $v$,

$$
\operatorname{LC}_{h}^{k+}(u, v) \text { is the complement of the connected component of } v \text { in } G \backslash \operatorname{LC}_{h}^{k+}(u) .
$$

Figure 6 illustrates a sublevel component and a sublevel set in $\mathbb{Z}^{d}$. In our applications sublevel sets are mostly used as a part of the definition of sublevel components, without a significant role of their own. To simplify our notation we write $\mathrm{LC}_{h}^{+}(u)$ for $\mathrm{LC}_{h}^{h(u)+}(u)$ and $\mathrm{LC}_{h}^{+}(u, v)$ for $\operatorname{LC}_{h}^{h(u)+}(u, v)$.

In the rest of the section we prove structure theorems for sublevel components of HHFs, mainly on $\mathbb{Z}^{d}$. In Section 4.1 we establish several basic properties of sublevel components. In Section 4.2 we show that sublevel components on $\mathbb{Z}^{d}$ are co-connected and boundary disjoint so that they satisfy the conditions of the pair-trichotomy (Theorem 3.2). In Section 4.3, we give a formula for computing the height difference between two vertices in terms of the sublevel components separating them. In Section 4.4 we show that sublevel components of quasi-periodic HHFs are translation respecting and hence satisfy the conditions of Theorem 3.4 and can be assigned a type. We conclude there that when $m \neq 0$, any $\mathrm{HHF}$ in $\mathrm{QP}_{m}$ has type-0 sublevel components. In Section 4.5 we introduce superlevel components and discuss their relationships with sublevel components. Finally, Section 4.6 gives a condition for two HHFs to share the same sublevel component. 


\subsection{Basic properties of sublevel components}

Let $G$ be either $\mathbb{Z}^{d}$ or $\mathbb{T}_{n}^{d}$. Let $h \in \operatorname{Hom}(G)$ and suppose $u, v \in G$ satisfy (4.1). Let

$$
U:=\operatorname{LC}_{h}^{k+}(u, v)
$$

The next proposition collects several basic properties of sublevel components of $h$.

Proposition 4.1. The sublevel component $U$ satisfies:

1. $u \in U$ and $v \notin U$.

2. $h(x)=k$ for all $x \in \partial_{\bullet} U$, and $h(x)=k+1$ for all $x \in \partial_{\circ} U$. In particular, $U$ is odd.

3. $U$ is co-connected.

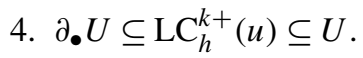

All of these properties are straightforward from the definition and we omit their proof.

In view of the second item of the proposition, we write, with a slight abuse of notation, $h\left(\partial_{\bullet} U\right)$ and $h\left(\partial_{\circ} U\right)$ for the common height of all vertices in $\partial_{\bullet} U$ and $\partial_{\circ} U$, respectively.

In the next corollary, we give useful criteria for containment relations between a connected set in $G$ and a sublevel component.

\section{Corollary 4.2. The sublevel component $U$ satisfies:}

- If $V \subseteq V(G)$ is connected and satisfies $v \in V, u \notin V$ and $h(w)>k$ for all $w \in \partial_{\bullet} V$, then $V \subseteq U^{c}$.

- If $V \subseteq V(G)$ is connected and satisfies $V \cap U \neq \varnothing, \partial_{\circ} U \subseteq V^{c}$, then $V \subseteq U$.

Proof. To get the first item, observe that, by definition of the $k$-sublevel set $\mathrm{LC}_{h}^{k+}(u)$ and the fact that an HHF changes by one between neighbors, $\partial_{\bullet} V \subset \operatorname{LC}_{h}^{k+}(u)^{c}$. As $\partial_{\bullet} V$ separates $V$ from $u$, we have $V \subset \operatorname{LC}_{h}^{k+}(u)^{c}$. Together with the fact that $V$ is a connected set containing $v$, the first item follows. The second item is straightforward and we omit its proof.

\subsection{Sublevel components on $\mathbb{Z}^{d}$}

Until the end of Section 4 we discuss the structure of the set of sublevel components of a single HHF on $\mathbb{Z}^{d}$. Throughout the rest of Section 4 , we denote by $h$ an arbitrary function in $\operatorname{Hom}\left(\mathbb{Z}^{d}\right)$. In the beginning of Section 4.4 we shall impose additional restrictions on $h$. Note that dependence on $h$ will often be implicit in our notation.

\section{Boundary disjointness}

The following proposition implies that sublevel components on $\mathbb{Z}^{d}$ satisfy the conditions of the pair trichotomy, Theorem 3.2.

Proposition 4.3. Distinct sublevel components of a function $h \in \operatorname{Hom}\left(\mathbb{Z}^{d}\right)$ are boundary disjoint.

Proof. Consider $U:=\operatorname{LC}_{h}^{k+}(u, v)$ and $V:=\operatorname{LC}_{h}^{\ell+}(x, y)$, where $k, \ell \in \mathbb{Z}$ and $u, v, x, y \in \mathbb{Z}^{d}$ satisfy $h(u) \leq k<h(v)$ and $h(x) \leq \ell<h(y)$. Observe that if $k \neq \ell$, the proposition holds trivially, by the second item of Proposition 4.1 and (3.1). We thus assume $k=\ell$. Suppose $U$ and $V$ are not boundary disjoint and let us show that this implies them being equal. From the second item of Proposition 4.1, and using (3.1), we get that there exists $e=\left(w_{1}, w_{2}\right) \in \partial U \cap \partial V$, such that $w_{1} \in \partial_{\bullet} U \cap \partial_{\bullet} V$. By the fourth item of Proposition 4.1 we have $w_{1} \in \operatorname{LC}_{h}^{k+}(u) \cap \operatorname{LC}_{h}^{k+}(x)$ and thus $\operatorname{LC}_{h}^{k+}(u)=$ $\operatorname{LC}_{h}^{k+}(x)$, by the definition of sublevel sets. Since $w_{2}$ is in the connected component of both $v$ and $y$ in $\mathbb{Z}^{d} \backslash \mathrm{LC}_{h}^{k+}(u)$, then these connected components are equal and we get $\operatorname{LC}_{h}^{k+}(u, v)=\operatorname{LC}_{h}^{k+}(x, y)$, as required.

From Proposition 4.3 we derive the following corollary. 

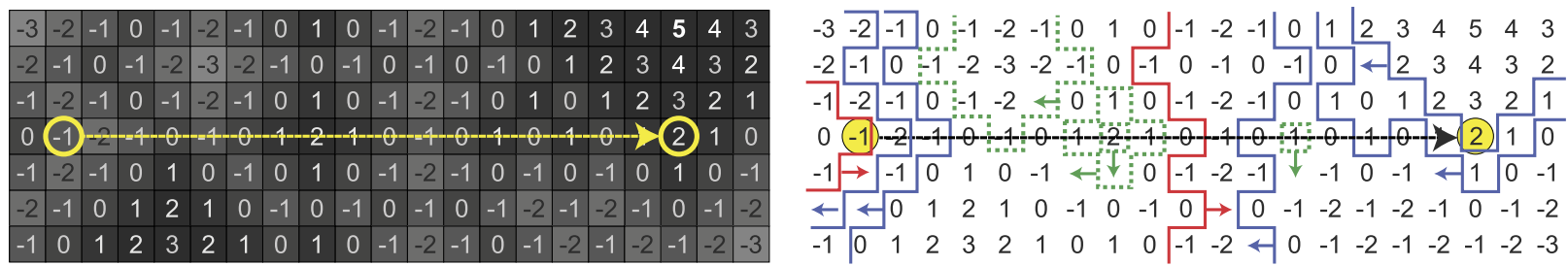

Fig. 7. Left: an HHF on $\mathbb{Z}^{d}$ and a path between two vertices $u$ and $v$ with height difference 3 . Right: the same HHF with the boundaries of all sublevel components intersecting the path. The arrow coming out of each boundary line points towards its sublevel component. The two non-dashed boundaries whose arrows points right are those of sublevel components containing $v$ and not containing $u$. The five non-dashed boundaries whose arrows points left are those of sublevel components containing $u$ and not containing $v$. The remaining dashed boundaries are those of sublevel components which contain either both $u$ and $v$ or neither. Proposition 4.6 shows that the height difference between $u$ and $v$ equals the difference between the number of non-dashed boundaries whose arrows point right and the number of those whose arrows point left.

Corollary 4.4. Every edge $(u, v) \in \mathbb{Z}^{d}$ is contained in the boundary of a unique sublevel component.

Proof. Assume WLOG that $h(v)=h(u)+1$. By definition, $(u, v) \in \partial \operatorname{LC}_{h}^{+}(u, v)$. By Proposition 4.3 no other sublevel component has $(u, v)$ in its edge boundary.

The next proposition shows that in $\mathbb{Z}^{d}$, the fact that $A$ is a sublevel component of $h$ depends only on a certain neighborhood of the boundary of $A$.

Proposition 4.5. Let $h_{1}, h_{2} \in \operatorname{Hom}\left(\mathbb{Z}^{d}\right)$ be two HHFs. Let $A$ be a sublevel component of $h_{1}$ and let $u \in \partial_{\bullet}$ A. Suppose there exists $S \supseteq \partial_{\bullet} A \cup \partial_{\circ} A$ satisfying that $h_{1}(w)=h_{2}(w)$ for all $w \in S$ and that $\mathrm{LC}_{h_{1}}^{+}(u) \cap S$ is a connected set. Then $A$ is also a sublevel component of $h_{2}$.

Proof. By our assumption $h_{1}(w)=h_{2}(w)$ for all $w \in S$, and by definition $h_{1}(w) \leq h_{1}(u)$ for all $w \in \mathrm{LC}_{h_{1}}^{+}(u)$. We get that $h_{2}(w) \leq h_{2}(u)$ for all $w \in \operatorname{LC}_{h_{1}}^{+}(u) \cap S$. Putting this together with our assumptions that $u \in \partial_{\bullet} A \subseteq S$, and that $\mathrm{LC}_{h_{1}}^{+}(u) \cap S$ is connected, we get that

$$
\mathrm{LC}_{h_{1}}^{+}(u) \cap S \subseteq \mathrm{LC}_{h_{2}}^{+}(u),
$$

by the definition of sublevel sets.

Next, let $v \in \partial_{\circ} A$ be such that $u \sim v$. Observe that by Corollary 4.4, we have $A=\operatorname{LC}_{h_{1}}^{+}(u, v)$. Let $U:=\operatorname{LC}_{h_{2}}^{+}(u, v)$. We shall show that $A=U$, establishing the proposition. By the fourth item of Proposition 4.1 we have that $\partial_{\bullet} A \subseteq$ $\mathrm{LC}_{h_{1}}^{+}(u)$ so that, using (4.2) and our assumption that $\partial_{\bullet} A \subseteq S$, we get that $\partial_{\bullet} A \subseteq \mathrm{LC}_{h_{2}}^{+}(u)$. Thus, using the fourth item of Proposition 4.1 again yields that

$$
\partial_{\bullet} A \subseteq U
$$

By our assumptions and Proposition 4.1, $A^{c}$ is connected and satisfies $v \in A^{c}, u \notin A^{c}$ and $h_{2}\left(\partial_{\circ} A\right)=h_{1}\left(\partial_{\circ} A\right)=$ $h_{2}(u)+1$. Thus, the first item of Corollary 4.2 implies that $A^{c} \subseteq U^{c}$. Thus, using (4.3) and the fact that $U^{c}$ is connected by Proposition 4.1, shows that $A^{c}=U^{c}$. Hence $U=A$ as we wanted to show.

\subsection{Expressing height differences in terms of sublevel components}

In this section we develop a formula expressing the difference between the height assigned to a pair of vertices $u$ and $v$ in terms of sublevel components. The formula is similar to the Newton-Leibniz formula in that it expresses the global height difference in terms of local increments. A visual depiction of this similarity is given in Figure 7.

Let $u, v \in \mathbb{Z}^{d}$. We define the set of sublevel components separating $u$ from $v$ by

$$
\mathcal{L}_{(u, v)}:=\left\{A: \exists u^{\prime}, v^{\prime}, k \text { s.t. } h\left(u^{\prime}\right) \leq k<h\left(v^{\prime}\right) \text { and } A=\operatorname{LC}_{h}^{k+}\left(u^{\prime}, v^{\prime}\right) \text { satisfies } u \in A, v \notin A\right\} .
$$


Proposition 4.6. Let $u, v \in \mathbb{Z}^{d} . \mathcal{L}_{(u, v)}$ is finite and ordered by inclusion. Furthermore, the following formula holds:

$$
h(v)-h(u)=\left|\mathcal{L}_{(u, v)}\right|-\left|\mathcal{L}_{(v, u)}\right| .
$$

Proof. Let $U, V$ be distinct elements of $\mathcal{L}_{(u, v)}$. We begin by showing that $\mathcal{L}_{(u, v)}$ is ordered by inclusion. By Proposition 4.1, $U$ and $V$ are co-connected and by Proposition 4.3 they are boundary disjoint. Thus, $U$ and $V$ satisfy the conditions of Theorem 3.2. By the definition of $\mathcal{L}_{(u, v)}$, we have $u \in U \cap V$ and $v \in U^{c} \cap V^{c}$. We deduce that either $U \subseteq V$ or $V \subseteq U$. As containment relations are transitive we deduce that $\mathcal{L}_{(u, v)}$ is ordered by inclusion.

To prove the remaining claims we use induction on the distance between $u$ and $v$. Indeed, the case $u=v$ is trivial. Assume that the proposition holds for every pair of vertices exactly at distance $\rho$ and suppose $u, v$ satisfy $\operatorname{dist}(u, v)=\rho+1$. Next, let $w$ be a vertex satisfying $w \sim u$ and $\operatorname{dist}(w, v)=\rho$. By our assumption

$$
h(v)-h(w)=\left|\mathcal{L}_{(w, v)}\right|-\left|\mathcal{L}_{(v, w)}\right|,
$$

and thus

$$
h(v)-h(u)=\left|\mathcal{L}_{(w, v)}\right|-\left|\mathcal{L}_{(v, w)}\right|+h(w)-h(u) .
$$

Suppose that $h(w)=h(u)+1$. Thus $U=\mathrm{LC}_{h}^{+}(u, w)$ is well defined. By Corollary 4.4, it is the only sublevel component containing $u$ and not containing $w$, and there is no sublevel component which contains $w$ and does not contain $u$. If $v \in U$, we get that $\mathcal{L}_{(u, v)}=\mathcal{L}_{(w, v)}$ and that $\mathcal{L}_{(v, w)}=\mathcal{L}_{(v, u)} \uplus\{U\}$. If $v \notin U$, we get that $\mathcal{L}_{(u, v)}=$ $\mathcal{L}_{(w, v)} \uplus\{U\}$ and that $\mathcal{L}_{(v, u)}=\mathcal{L}_{(v, w)}$. In either case, by (4.5),

$$
h(v)-h(u)=\left|\mathcal{L}_{(u, v)}\right|-\left|\mathcal{L}_{(v, u)}\right| .
$$

The case $h(u)=h(w)+1$ follows similar lines.

\subsection{Sublevel components of quasi-periodic HHFs on $\mathbb{Z}^{d}$}

In this subsection we impose the further requirement that $h$ is quasi-periodic, that is, that $h \in \mathrm{QP}_{m}$ for some $m \in \mathbb{Z}^{d}$. We show that sublevel components of such functions are translation respecting and are thus classified into types according to the translation trichotomy, Theorem 3.4. We conclude that when $m \neq 0$, any such function has a type- 0 sublevel component.

The first property we observe is that the set of sublevel components of $h$ is invariant under translations in $n \mathbb{Z}^{d}$.

Proposition 4.7. Let $k \in \mathbb{Z}$ and $u, v \in \mathbb{Z}^{d}$ be such that $h(u) \leq k<h(v)$. For any $x \in n \mathbb{Z}^{d}$ we have $\operatorname{LC}_{h}^{\left(k+\delta_{x}\right)+}(u+$ $x, v+x)=\operatorname{LC}_{h}^{k+}(u, v)+x$ where $\delta_{x}:=h(x)-h(\mathbf{0})$.

The proposition follows directly from the definition of sublevel component and quasi-periodic function and we omit its proof. A consequence of this proposition is the following.

Corollary 4.8. Every sublevel component of $h$ is translation respecting.

To see this recall that sublevel components are co-connected by the third item of Proposition 4.1, and apply Proposition 4.3 together with Proposition 4.7.

Corollary 4.8 tells us that sublevel components of quasi-periodic HHFs may be assigned a type, as in Section 3.1. We remark that it is possible that a sublevel component $A$ will be invariant under all translations in $n \mathbb{Z}^{d}$, in which case we follow the convention of Section 3 by assigning to it both type -1 and type 1 . However, we note that this cannot happen when the slope $m$ of the quasi-periodic function $h$ is non-zero, the case of most interest to us, as follows from Proposition 4.7 and the second item of Proposition 4.1.

The following corollary provides a formula for the height difference between translates of a type 0 sublevel component. 
Corollary 4.9. Let $U$ be a type 0 sublevel component of $h$ with minimal translation $\Delta$ and write $\delta:=h\left(\partial_{\bullet} U+\Delta\right)-$ $h\left(\partial_{\bullet} U\right)$. Then for any $z \in n \mathbb{Z}^{d}$ we have

$$
h\left(\partial_{\bullet}(U+z)\right)-h\left(\partial_{\bullet} U\right)=\delta \cdot o_{U}(U+z),
$$

where $o_{U}$ is the order function on translates of $U$, given by Theorem 3.4.

This is an immediate consequence of Theorem 3.4 and the fact that $h$ is quasi-periodic.

The next proposition establishes a duality between $\mathcal{L}_{(u, v)}$ and $\mathcal{L}_{(v, u)}$ when $u-v \in n \mathbb{Z}^{d}$.

Proposition 4.10. Let $u, z \in \mathbb{Z}^{d}$ with $z \neq \mathbf{0}$. If $A \in \mathcal{L}_{(u, u+n z)}$ has $\operatorname{Type}(A) \neq 0$ then the type of $A$ is uniquely defined and

$$
A+\operatorname{Type}(A) \cdot n z \in \mathcal{L}_{(u+n z, u)} .
$$

Proof. Let $A \in \mathcal{L}_{(u, u+n z)}$ for $u, z \in \mathbb{Z}^{d}$, and observe that since $u \in A$ but $u+n z \notin A$ we have that $A$ is not invariant under translations in $n \mathbb{Z}^{d}$ and hence Type $(A)$ is uniquely defined. Suppose that Type $(A) \neq 0$, i.e., Type $(A) \in\{-1,1\}$. Recall that by definition, $u \in A$ and $u+n z \notin A$. Since $A$ is a sublevel component then, by Proposition 4.7, $A \pm n z$ are also sublevel components. Both are distinct from $A$ since $u+n z \in A+n z$ and $u \notin A-n z$. If Type $(A)=1$, then by the trichotomy of Theorem 3.4, $u \in A$ implies that $u \notin A+n z$. Similarly if $\operatorname{Type}(A)=-1$, then by the same trichotomy $u+n z \notin A$ implies $u+n z \in A-n z$. In either case the proposition holds.

An important corollary of the above proposition is the following:

Corollary 4.11. If $h \in \mathrm{QP}_{m}$ for $m=\left(m_{1}, \ldots, m_{d}\right)$ satisfying $m_{1}>0$, then there exists a sublevel component of type 0 which contains $\mathbf{0}$ and does not contain ne 1 .

Proof. Suppose to the contrary that every sublevel component in $\mathcal{L}_{\left(\mathbf{0}, n e_{1}\right)}$ is either of type 1 or of type -1 . By Proposition 4.10 we get that $\left|\mathcal{L}_{\left(\mathbf{0}, n e_{1}\right)}\right| \leq\left|\mathcal{L}_{\left(n e_{1}, \mathbf{0}\right)}\right|$. By Proposition 4.6 this implies $h\left(n e_{1}\right) \leq h(\mathbf{0})$, in contradiction to our premise. Here, we have also used the fact that the type of a sublevel component is preserved under translation, thus distinct sublevel components $A \in \mathcal{L}_{\left(\mathbf{0}, n e_{1}\right)}$ are mapped to distinct sublevel components in $\mathcal{L}_{\left(n e_{1}, \mathbf{0}\right)}$ by the mapping $A \mapsto A+\operatorname{Type}(A) \cdot n e_{1}$.

\subsection{Superlevel components and sublevel components of type 0}

In the construction of our embedding (in Section 5) we make use of superlevel components. These are counterparts of sublevel components, in which the role of the sublevel set is replaced by a superlevel set. The main reason that superlevel components are necessary for our construction is that in order to guarantee invertibility of the mapping $\Psi_{m}$, we wish to define it through an exploration process in a region which is left unchanged by the mapping. Exploration in one direction is done by finding sublevel components while exploration in the other direction is done through superlevel components.

While superlevel components could be defined in an analogous way to that of sublevel components, as given at the beginning of Section 4 , we rather define them through a duality.

Definition 4.12. For any $u, v \in \mathbb{Z}^{d}$ and $k \in \mathbb{Z}$ satisfying $h(v)<k \leq h(u)$, we define

$$
\operatorname{LC}_{h}^{k-}(u, v):=\operatorname{LC}_{-h}^{(-k)+}(u, v) .
$$

This definition allows us to apply propositions dealing with sublevel components to superlevel components. For instance, combining the definition with Corollary 4.8 and Theorem 3.4 we can assign a type to every superlevel component. In addition, by Proposition 4.1, a superlevel component $U=\mathrm{LC}_{h}^{k-}(u, v)$ satisfies $h(x)=k$ for all $x \in$ $\partial_{\bullet} U$, and $h(x)=k-1$ for all $x \in \partial_{\circ} U$. However, to avoid confusion, we remark that the complement of a superlevel component is not necessarily a sublevel component. 
The next lemma shows that certain sublevel and superlevel components which are "sandwiched" between two type 0 sublevel components must also be of type 0 .

Lemma 4.13. Let $U \subsetneq W$ be a pair of type 0 sublevel components, such that $h\left(\partial_{\circ} U\right)<h\left(\partial_{\circ} W\right)$ and let $u \in \partial_{\bullet} U$, $w \in \partial_{\bullet} W$ and $k \in \mathbb{Z}$. Then:

- If $h(u) \leq k<h(w)$ then $V_{+}:=\operatorname{LC}_{h}^{k+}(u, w)$ is a sublevel component of type 0 , satisfying $U \subseteq V_{+} \subsetneq W$.

- If $h(u)<k \leq h(w)$ then $V_{-}:=\left(\operatorname{LC}_{h}^{k-}(w, u)\right)^{c}$ satisfies that $\left(V_{-}\right)^{c}$ is a superlevel component of type 0 and $U \subseteq$ $V_{-} \subsetneq W$.

Proof. We start by proving the first item and let $V_{+}$be as in the lemma. We first show that

$$
U=\mathrm{LC}_{h}^{+}(u, w) .
$$

By our assumptions, $U=\mathrm{LC}_{h}^{h(u)+}\left(u^{\prime}, v^{\prime}\right)$ for some $u^{\prime}, v^{\prime}$. By the fourth item of Proposition 4.1 we have $\mathrm{LC}_{h}^{+}(u)=$ $\operatorname{LC}_{h}^{h(u)+}\left(u^{\prime}\right)$. Next, $w \notin U$ since $U \subsetneq W$ and $U$ and $W$ are boundary disjoint by Proposition 4.3. Hence (4.6) follows.

Now observe that by applying (4.6), Proposition 4.1 and the first item of Corollary 4.2 to $U$ and $\left(V_{+}\right)^{c}$, we get that $\left(V_{+}\right)^{c} \subseteq U^{c}$, i.e., $U \subseteq V_{+}$. Similarly, by Proposition 3.1,

$\partial_{\bullet} W \cup \partial_{\circ} W$ is a connected set containing $w$, whose vertices are of height greater than $k$,

and hence $u \notin \partial_{\bullet} W \cup \partial_{\circ} W$. Thus, applying (4.7) and the first item of Corollary 4.2, we deduce that $\left(\partial_{\bullet} W \cup \partial_{\circ} W\right) \subseteq V_{+}^{c}$. We can now use the second item of Corollary 4.2 to deduce that $V_{+} \subseteq W$. Consequently, $U \subseteq V_{+} \subsetneq W$, where we have used also that $w \in W \backslash V_{+}$. It remains to show that $V_{+}$is of type 0 . All that we need in order to draw this conclusion from Proposition 3.6 is to show that $\left|T_{W}\right|,\left|T_{V_{+}}\right|,\left|T_{U}\right|>1$. To see this first observe that since Type $(U)=\operatorname{Type}(W)=$ 0 , we have by definition $\left|T_{W}\right|,\left|T_{U}\right|>1$. By Proposition 3.7 there exists some $\Delta \in n \mathbb{Z}^{d}$ satisfying $(U+\Delta) \cap\left(V_{+}\right)^{c} \neq$ $\varnothing$ while $U+\Delta \subseteq V_{+}+\Delta$. We deduce that $\left|T_{V_{+}}\right|>1$, so that $V_{+}$is of type 0 .

The second item is proved similarly. Let $V_{-}$be as in the lemma. By the definition of superlevel components and Proposition 4.1, we have that $\left(V_{-}\right)^{c}$ is connected, $u \notin\left(V_{-}\right)^{c}, w \in\left(V_{-}\right)^{c}$ and $h\left(\partial_{\circ} V_{-}\right)>h(u)$. Applying (4.6) and the first item of Corollary 4.2 to $\left(V_{-}\right)^{c}$ we deduce that $\left(V_{-}\right)^{c} \subseteq U^{c}$, i.e., $U \subseteq V_{-}$.

Applying (4.7), the definition of a superlevel component, and the fourth item of Proposition 4.1 we get that $\partial_{\bullet} W \cup$ $\partial_{\circ} W \subseteq\left(V_{-}\right)^{c}$, as it is contained in the corresponding superlevel set. We deduce that $V_{-}$is a connected set satisfying $u \in V_{-}$and $\partial_{\circ} W \subseteq\left(V_{-}\right)^{c}$. Therefore by the second item of Corollary 4.2, we have $V_{-} \subseteq W$. Consequently, $U \subseteq V_{-} \subsetneq$ $W$, where we have used also that $w \in W \backslash V_{-}$. It remains to show that $V_{-}$is of type 0 . All that we need in order to draw this conclusion from Proposition 3.6 is to show that $\left|T_{V_{-}}\right|>1$. This is done in exactly the same way as in the proof of the first part of the lemma.

\subsection{Locality property of sublevel components}

We conclude Section 4 with a useful criterion for applying Proposition 4.5, to show that two HHFs in Hom $\left(\mathbb{Z}^{d}\right)$ share the same sublevel component.

Proposition 4.14. Let $h_{1}, h_{2} \in \operatorname{Hom}\left(\mathbb{Z}^{d}\right)$ be two HHFs and let $A$ be a sublevel component of $h_{1}$. Suppose that

$$
h_{1}(w)=h_{2}(w) \text { for all } w \in A^{+} \backslash B^{-},
$$

for some $B \subsetneq A$ which is either a sublevel component of $h_{1}$ or the complement of a superlevel component of $h_{1}$. Then $A$ is also a sublevel component of $h_{2}$.

Proof. Let $u \in \partial_{\bullet} A$. Let $v \in \partial_{\circ} A$ be such that $u \sim v$. By Corollary 4.4,

$$
A=\mathrm{LC}_{h_{1}}^{+}(u, v) \text {. }
$$


Let us show that $u \notin B$. Suppose to the contrary that $u \in B$. Hence $u \in \partial_{\bullet} B$ by our assumption that $B \subsetneq A$. Then, by Proposition 4.1 and the definition of superlevel component, $B^{c}$ is a connected set satisfying $v \in B^{c}$ and satisfying $h_{1}\left(\partial_{\circ} B\right)=h_{1}(u)+1>h_{1}\left(\partial_{\bullet} A\right)$. Thus, by the first item of Corollary 4.2, we have that $B^{c} \subseteq A^{c}$. However, this contradicts the fact that $B \subsetneq A$.

We continue by considering separately two cases. First, assume that

$$
\text { either } h_{1}\left(\partial_{\bullet} B\right)>h_{1}(u) \quad \text { or } \quad h_{1}\left(\partial_{\circ} B\right)>h_{1}(u) \text {. }
$$

Since $u \notin B$, the definition of $\operatorname{LC}_{h_{1}}^{+}(u)$ and the assumption (4.10) imply that $\operatorname{LC}_{h_{1}}^{+}(u) \cap B=\varnothing$. Now, Proposition 4.1 and (4.9) imply that $\operatorname{LC}_{h_{1}}^{+}(u) \subseteq A$. Thus, by (4.8), $h_{1}(w)=h_{2}(w)$ for all $w \in\left(\operatorname{LC}_{h_{1}}^{+}(u)\right)^{+}$. Hence the definition of sublevel set yields that $\operatorname{LC}_{h_{1}}^{+}(u)=\operatorname{LC}_{h_{2}}^{+}(u)$, which, in turn, implies that $\operatorname{LC}_{h_{1}}^{+}(u, v)=\operatorname{LC}_{h_{2}}^{+}(u, v)$. Thus, recalling (4.9), $A$ is also a sublevel component of $h_{2}$.

Second, let us assume that (4.10) does not hold. That is, that

$$
h_{1}\left(\partial_{\bullet} B\right) \leq h_{1}(u) \quad \text { and } \quad h_{1}\left(\partial_{\circ} B\right) \leq h_{1}(u) .
$$

Denote $S:=A^{+} \backslash B^{-}$. Recalling (4.8) and observing that

$$
A^{+} \backslash A^{-}=\partial_{\bullet} A \cup \partial_{\circ} A \subseteq S,
$$

all that we need to show in order to apply Proposition 4.5 and derive the proposition, is that

$$
\mathrm{LC}_{h_{1}}^{+}(u) \cap S \text { is connected. }
$$

Observe that, as $\operatorname{LC}_{h_{1}}^{+}(u) \subseteq \operatorname{LC}_{h_{1}}^{+}(u, v)=A$ by Proposition 4.1, we have

$$
\operatorname{LC}_{h_{1}}^{+}(u) \cap S=\mathrm{LC}_{h_{1}}^{+}(u) \backslash B^{-} .
$$

Let $H_{0} \uplus H_{1}$ be a non-trivial partition of $\mathrm{LC}_{h_{1}}^{+}(u) \backslash B^{-}$. Assume for the sake of obtaining a contradiction that there is no edge in $\mathbb{Z}^{d}$ connecting $H_{0}$ and $H_{1}$ (that is an edge between a vertex in $H_{0}$ and a vertex in $H_{1}$ ). Since $H_{0} \uplus$ $H_{1} \uplus\left(\mathrm{LC}_{h_{1}}^{+}(u) \cap B^{-}\right)=\mathrm{LC}_{h_{1}}^{+}(u)$, and $\mathrm{LC}_{h_{1}}^{+}(u)$ is a connected set, there must be an edge of $\mathbb{Z}^{d}$ connecting $H_{0}$ and $\mathrm{LC}_{h_{1}}^{+}(u) \cap B^{-}$, and an edge of $\mathbb{Z}^{d}$ connecting $H_{1}$ and $\mathrm{LC}_{h_{1}}^{+}(u) \cap B^{-}$. The existence of these edges implies that

$$
\begin{aligned}
& \left(B^{+} \backslash B^{-}\right) \cap H_{0} \neq \varnothing \quad \text { and } \\
& \left(B^{+} \backslash B^{-}\right) \cap H_{1} \neq \varnothing .
\end{aligned}
$$

In particular,

$$
\left(B^{+} \backslash B^{-}\right) \cap\left(\mathrm{LC}_{h_{1}}^{+}(u) \backslash B^{-}\right) \neq \varnothing .
$$

By Proposition 3.1, we have that

$$
B^{+} \backslash B^{-} \text {is a connected set. }
$$

Observe that $\mathrm{LC}_{h_{1}}^{+}(u)$ is a connected component of $\left\{w: h_{1}(w) \leq h_{1}(u)\right\}$, and, by (4.11), $B^{+} \backslash B^{-} \subseteq\left\{w: h_{1}(w) \leq\right.$ $\left.h_{1}(u)\right\}$. Thus, using (4.14) and (4.15) we may deduce that

$$
\left(B^{+} \backslash B^{-}\right) \subseteq \mathrm{LC}_{h_{1}}^{+}(u) \backslash B^{-}=H_{0} \cup H_{1} .
$$

Putting together (4.16) and (4.13) we get that $H_{0} \uplus H_{1}$ induces a non-trivial partition on $B^{+} \backslash B^{-}$that is not crossed by any edge. Since this contradicts (4.15), we deduce that (4.12) holds. 


\section{Proof of the embedding theorem}

In this section we use the theory developed in the previous sections to prove Theorem 2.3. In Section 5.1 we present a one-to-one mapping from $\mathrm{QP}_{m}$, the set of quasi-periodic HHFs with slope $m$, to $\mathrm{QP}_{\mathbf{0}}$, the set of periodic HHFs. In Section 5.2 we prove Theorem 2.3 using a probabilistic bound taken from [18] and an auxiliary lemma. This lemma, which relates the boundaries of sublevel components in $\mathrm{QP}_{\mathbf{0}}$ with the boundaries of sublevel components of HHFs in $\operatorname{Hom}\left(\mathbb{T}_{n}^{d}\right)$, is then proved in Section 5.3.

\subsection{Mapping quasi-periodic to periodic}

Throughout this section we fix some $m=\left(m_{1}, \ldots, m_{d}\right) \in 6 \mathbb{Z}^{d}$ such that

$$
m_{1}>0 \text { and } \mathrm{QP}_{m} \neq \varnothing .
$$

We also fix $h \in \mathrm{QP}_{m}$. With the structural results of Sections 3 and 4 in our toolkit, we are ready to construct $\Psi_{m}$, our one-to-one mapping from $\mathrm{QP}_{m}$ into $\mathrm{QP}_{\mathbf{0}}$. We start by defining three sets, $U_{0}, V_{0}$ and $W_{0}$. The definition relies on the fact that by Corollary 4.8, sublevel and superlevel components of $h$ are translation respecting and can therefore be assigned a type by Theorem 3.4. The first and the third sets will be used to construct $\Psi_{m}$. The second set will be used in Section 5.2 to show that the image of $\Psi_{m}$ is small. Proposition 5.1 below shows that the three sets are well defined.

In the following definition, and throughout the entire section, we say that a set $S \subset \mathbb{Z}^{d}$ is the minimal set with a given property, if $S$ is contained in every other set with that property.

- $W_{0}=W_{0}(h)$ is the minimal type 0 sublevel component satisfying

$$
\mathbf{0} \in W_{0} \quad \text { and } n e_{1} \notin W_{0} .
$$

We let $\Delta$ be a minimal translation of $W_{0}$ as in Theorem 3.4. We choose $\Delta$ in some prescribed manner, e.g., as the minimal translation which is first in lexicographic order among the minimal translations with smallest $\ell_{1}$ norm. Write

$$
\delta:=h(\Delta)-h(\mathbf{0})=h(\Delta) .
$$

- $V_{0}=V_{0}(h)$ is the maximal type 0 sublevel component satisfying

$$
h\left(\partial_{\bullet} V_{0}\right)=h\left(\partial_{\bullet} W_{0}\right)-1, \quad W_{0}-\Delta \subseteq V_{0} \subseteq W_{0}, \quad \mathbf{0} \notin V_{0} \quad \text { and } \quad-n e_{1} \in V_{0} .
$$

- $U_{0}=U_{0}(h)$ is defined by the property that its complement $U_{0}^{c}$ is the minimal type 0 superlevel component such that

$$
h\left(\partial_{\bullet} U_{0}\right)=h\left(\partial_{\circ} W_{0}\right)-\delta / 2, \quad W_{0}-\Delta \subseteq U_{0} \subseteq W_{0}, \quad \mathbf{0} \notin U_{0} \quad \text { and } \quad-n e_{1} \in U_{0} .
$$

We remark that the third and fourth properties in (5.2) and (5.3) in fact follow from the first two properties. Nonetheless, to simplify our arguments we include them as part of the definition. The sets $U_{0}, V_{0}$ and $W_{0}$ of a certain $h \in \mathrm{QP}_{(6,0)}$ are illustrated in Figure 8 .

Proposition 5.1. $W_{0}, V_{0}$ and $U_{0}$ are well-defined, and satisfy

$$
W_{0}-\Delta \subsetneq U_{0} \subsetneq V_{0} \subsetneq W_{0}
$$

Proof. For brevity we write $U, V$ and $W$, for $U_{0}, V_{0}$ and $W_{0}$ respectively. We begin by showing that $W$ is well defined. Write $\mathcal{W}$ for the set of type 0 sublevel components which contain $\mathbf{0}$ and do not contain $n e_{1}$. Recalling (4.4) we observe that $\mathcal{W} \subseteq \mathcal{L}_{\left(0, n e_{1}\right)}$. Thus, by Proposition $4.6, \mathcal{W}$ is ordered by inclusion and finite. By Corollary 4.11, $\mathcal{W} \neq \varnothing$, and thus $W$, the minimal element of $\mathcal{W}$, is well defined.

Next, towards showing that $V$ is well defined, we write $\mathcal{V}$ for the set of type 0 sublevel components $V^{\prime}$ satisfying $h\left(\partial_{\bullet} V^{\prime}\right)=h\left(\partial_{\bullet} W\right)-1, W-\Delta \subsetneq V^{\prime} \subsetneq W, \mathbf{0} \notin V^{\prime}$ and $-n e_{1} \in V^{\prime}$. We observe that $\mathcal{V} \subseteq \mathcal{L}_{\left(-n e_{1}, 0\right)}$, and thus by 

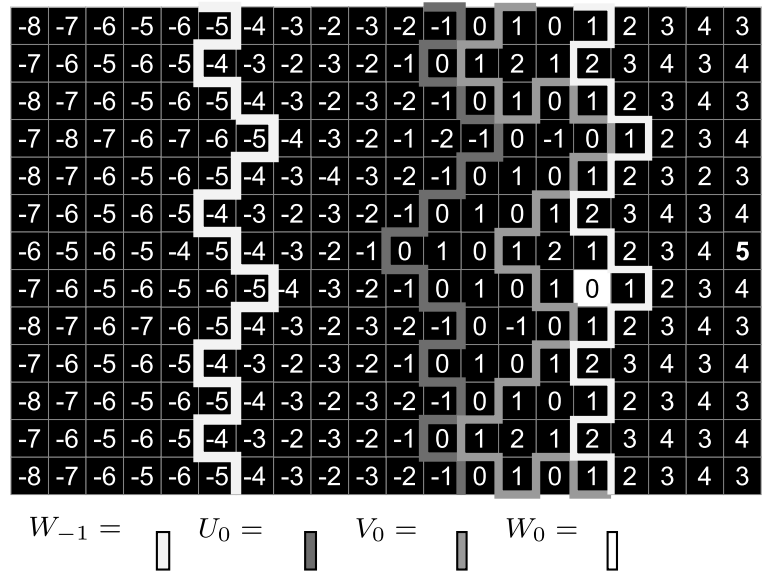

Fig. 8. The boundaries of $U_{0}, V_{0}, W_{0}$ and $W_{-1}=W_{0}-\Delta$ for $\Delta=n e_{1}$ and $\delta=6$. The sets themselves are in all cases to the left of the boundary. 0 is marked in white.

Proposition 4.6, $\mathcal{V}$ is ordered by inclusion and finite. To derive the existence of $V$, all that remains is to show that $\mathcal{V} \neq \varnothing$.

To see that $\mathcal{V} \neq \varnothing$, we make some observations about $\Delta$ and $\delta$. Since $h \in \mathrm{QP}_{m}, m \in 6 \mathbb{Z}^{d}$ and $\Delta \in n \mathbb{Z}^{d}$, it follows that

$$
\delta \equiv 0 \quad(\bmod 6)
$$

Since $W$ is of type $0, \mathbf{0} \in W$ and $n e_{1} \notin W$ we get that $W \subsetneq W+n e_{1}$ and therefore, by Theorem 3.4,

$$
W+n e_{1}=W+k \Delta \quad \text { for some positive } k .
$$

We deduce, using Proposition 4.7, that $h\left(\partial_{\bullet} W+n e_{1}\right)=h\left(\partial_{\bullet} W\right)+h\left(n e_{1}\right)=h\left(\partial_{\bullet} W\right)+m_{1}$, and therefore that $m_{1}=k \delta$ In particular, since $m_{1}>0$, we see that

$$
\delta \geq 6 .
$$

By subtracting $n e_{1}$ and $k \Delta$ from both sides of (5.6) we have that $W-n e_{1}=W-k \Delta$. Thus, recalling that $0 \in W$ and $W-k \Delta \subseteq W-\Delta$, we obtain that

$$
-n e_{1} \in W-\Delta \text {. }
$$

By Proposition 4.7 and (5.7) we get that $W-\Delta$ is a sublevel component satisfying $h\left(\partial_{\circ}(W-\Delta)\right)=h\left(\partial_{\circ} W\right)-\delta \leq$ $h\left(\partial_{\circ} W\right)-6$. Thus, the first item of Lemma 4.13 guarantees the existence of a type 0 sublevel component $V^{\prime}$ satisfying $h\left(\partial_{\bullet} V^{\prime}\right)=h\left(\partial_{\bullet} W\right)-1, W-\Delta \subsetneq V^{\prime} \subsetneq W$. Since $-n e_{1} \in W-\Delta$ by (5.8) we get that $-n e_{1} \in V^{\prime}$. By the minimality of $W$, we get that $\mathbf{0} \notin V^{\prime}$ implying that $V^{\prime} \in \mathcal{V}$ so that $\mathcal{V} \neq \varnothing$.

To show that $U$ is well defined, we write $\mathcal{U}$ for the set containing all $U^{\prime}$ such that $\left(U^{\prime}\right)^{c}$ is a type 0 superlevel component such that $h\left(\partial_{\bullet} U^{\prime}\right)=h\left(\partial_{\circ} W\right)-\delta / 2, W-\Delta \subseteq U^{\prime} \subseteq W, \mathbf{0} \notin U^{\prime}$ and $-n e_{1} \in U^{\prime}$. Recalling Definition 4.12 of superlevel sets we use Proposition 4.6 to deduce that the set of superlevel sets containing $\mathbf{0}$ and not containing $-n e_{1}$ is finite and ordered by inclusion, and therefore $\mathcal{U}$ is also finite and ordered by inclusion. All that remains in order to deduce the existence of $U$ is to show that $\mathcal{U} \neq \varnothing$.

This time we apply (5.7) and the second item of Lemma 4.13, to $h, V$ and $W-\Delta$, to show the existence of $U^{\prime}$ satisfying that $\left(U^{\prime}\right)^{c}$ is a superlevel component of type $0, W-\Delta \subseteq U^{\prime} \subsetneq V$ and $h\left(\partial_{\bullet} U^{\prime}\right)=h\left(\partial_{\circ} W\right)-\delta / 2$. Since $\mathbf{0} \notin V$ by definition and $-n e_{1} \in W-\Delta$ by (5.8) we get that $\mathbf{0} \notin U^{\prime}$ and $-n e_{1} \in U^{\prime}$. Thus $U^{\prime} \in \mathcal{U}, \mathcal{U} \neq \varnothing$ so that $U$ is well defined.

To conclude the proof we must show that $U \subsetneq V$ as with the definitions of $U$ and $V$ this will imply (5.4). Let $u \in$ $\partial_{\bullet} U, w \in \partial_{\diamond} W$ and write $V^{\prime}=\operatorname{LC}_{h}^{\left(h\left(\partial_{\bullet} W\right)-1\right)+}(u, w)$. Our goal is to show that $V^{\prime} \in \mathcal{V}$. By the first item of Corollary 4.2 
applied to $V^{\prime}$ and $W^{c}$ we have $V^{\prime} \subseteq W$. Since $U$ is co-connected, by Proposition 3.1 we have that $\partial_{\bullet} U \cup \partial_{\circ} U$ is a connected set of vertices. Moreover, $h\left(\partial_{\bullet} U\right), h\left(\partial_{\circ} U\right) \leq h\left(\partial_{\circ} W\right)-\delta / 2+1 \leq h\left(\partial_{\bullet} W\right)-1$. Therefore $\partial_{\bullet} U \cup \partial_{\circ} U \subseteq$ $\operatorname{LC}_{h}^{\left(h\left(\partial_{\bullet} W\right)-1\right)+}(u)$ and since $w \notin U$ we have $U \subseteq V^{\prime}$ and hence that $-n e_{1} \in \bar{V}^{\prime}$. Since $W-\Delta \subsetneq V^{\prime} \subsetneq W$ and since $\bar{W}$ is of type 0 , we have by Proposition 3.6 that $V^{\prime}$ is of type 0 . By the minimality of $W$ we get that $0 \notin V^{\prime}$. Thus $V^{\prime} \in \mathcal{V}$. Since $U \subsetneq V^{\prime}$, and since $V^{\prime} \subseteq V$ by the maximality of $V$, we obtain (5.4) as required.

For $i \in \mathbb{Z}$, we write

$$
U_{i}:=U_{0}+i \Delta, \quad V_{i}:=V_{0}+i \Delta \quad \text { and } \quad W_{i}:=W_{0}+i \Delta .
$$

Proposition 5.2. For every $z \in n \mathbb{Z}^{d}$ and $i \in \mathbb{Z}$ the following are equivalent:

- $U_{0}+z=U_{i}$,

- $V_{0}+z=V_{i}$,

- $W_{0}+z=W_{i}$.

Proof. We begin by showing that $U_{0}, V_{0}$ and $W_{0}$ all have $\Delta$ as a minimal translation. For $W_{0}$, this is the case by the definition of $\Delta$. We now show this for $V_{0}$. The proof for $U_{0}$ is similar. Let $\Delta_{V}$ be a minimal translation of $V_{0}$. Since

$$
V_{0}-\Delta \subsetneq W_{0}-\Delta \subsetneq V_{0} \subsetneq W_{0}
$$

by (5.4), we have $V_{0}-k \Delta_{V}=V_{0}-\Delta$ for some integer $k \geq 1$. We need to show that $k=1$. By Proposition 3.3, we have

$$
\operatorname{dist}\left(V_{0}-\Delta_{V}, W_{0}^{c}\right)>\operatorname{dist}\left(V_{0}, W_{0}^{c}\right)=\operatorname{dist}\left(V_{0}-\Delta_{V}, W_{0}^{c}-\Delta_{V}\right) .
$$

We deduce that $W_{0}-\Delta_{V} \subsetneq W_{0}$, and thus $W_{0}-\Delta_{V} \subseteq W_{0}-\Delta$ (by the minimality of $\Delta$ ). Suppose to the contrary that $W_{0}-\Delta_{V} \subsetneq W_{0}-\Delta$. Since $\Delta$ is a minimal translation of $W_{0}$, we get that

$$
V_{0}-\Delta_{V} \subsetneq W_{0}-\Delta_{V} \subseteq W_{0}-2 \Delta \subsetneq V_{0}-\Delta,
$$

contradicting the minimality of $\Delta_{V}$. We conclude that $W_{0}-\Delta=W_{0}-\Delta_{V}$. From this, using (5.4) again, we have that

$$
V_{0}-2 \Delta_{V} \subsetneq W_{0}-2 \Delta_{V}=W_{0}-2 \Delta \subsetneq V_{0}-\Delta=V_{0}-k \Delta_{V}
$$

so that $k \leq 1$, implying that $k=1$ as we wanted to show.

Fix $z \in n \mathbb{Z}^{d}$. Since $U_{0}, V_{0}$ and $W_{0}$ are of type 0 with $\Delta$ as a minimal translation, there exist $i, j, k$ for which $U_{0}+z=U_{i}, V_{0}+z=V_{j}, W_{0}+z=W_{k}$. Translating (5.4) by $z$, we have

$$
W_{k-1} \subsetneq U_{i} \subsetneq V_{j} \subsetneq W_{k} .
$$

However, (5.4) and (5.9) imply that

$$
W_{-1} \subsetneq U_{0} \subsetneq V_{0} \subsetneq W_{0} \subsetneq U_{1} \subsetneq V_{1} .
$$

Hence we conclude from (5.10) and the fact that $\left(U_{i}\right),\left(V_{i}\right)$ and $\left(W_{i}\right)$ are ordered by inclusion that

$$
k-1<i \leq j \leq k
$$

and therefore that $i=j=k$.

We define the mapping $\Psi_{m}: \mathrm{QP}_{m} \rightarrow \mathrm{QP}_{\mathbf{0}}$ by

$$
\Psi_{m}(h)(v):= \begin{cases}h(v-i \Delta)=h(v)-i \delta, & v \in W_{i} \backslash U_{i} \text { for some } i \in \mathbb{Z}, \\ 2 h\left(\partial_{\circ} W_{0}\right)-h(v-i \Delta)=2 h\left(\partial_{\circ} W_{0}\right)-h(v)+i \delta, & v \in U_{i+1} \backslash W_{i} \text { for some } i \in \mathbb{Z} .\end{cases}
$$




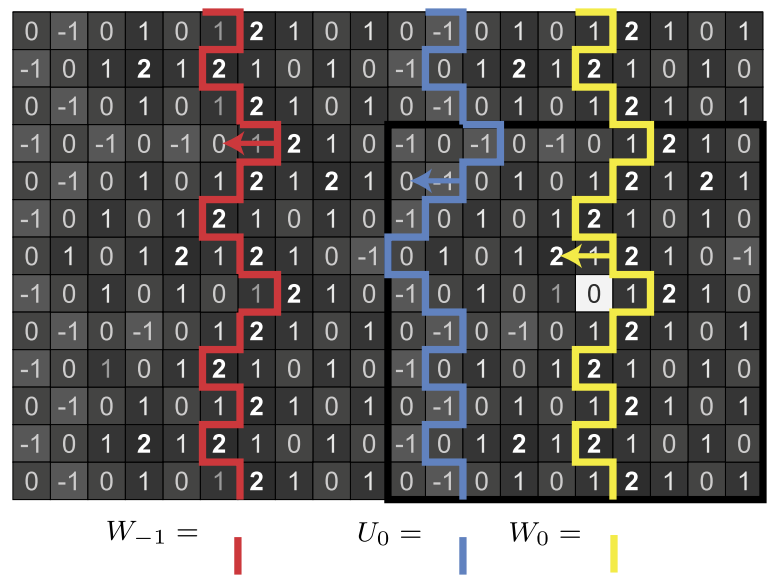

Fig. 9. The image through $\Psi$ of the HHF illustrated in Figure 8. The boundaries of $U_{0}, W_{0}$ and $W_{-1}$ are highlighted to allow the reader to follow the behavior of $\Psi$ in different regions. $\mathbf{0}$ is marked in white.

The remainder of the section is dedicated to showing that $\Psi_{m}$ is well defined and has the required properties.

By Theorem 3.4, for every $i \in \mathbb{Z}$ we have $W_{i} \subsetneq W_{i+1}$. Thus, applying Proposition 3.7 to $W_{0}$, we have that every $v \in \mathbb{Z}^{d}$ belongs to exactly one set of the form $W_{i+1} \backslash W_{i}$. Hence $\Psi_{m}(h)(v)$ is defined for every $v \in \mathbb{Z}^{d}$. The image through $\Psi$ of the HHF illustrated in Figure 8 is depicted in Figure 9.

By definition, $\Psi_{m}(h)$ is $\Delta$-periodic, i.e., it satisfies $\Psi_{m}(h)(v)=\Psi_{m}(h)(v+\Delta)$ for every $v \in \mathbb{Z}^{d}$. Thus to understand $\Psi_{m}(h)$ it suffices to understand its values on $v \in W_{0} \backslash W_{-1}$. As a first step to this end we point out that on the region $W_{0} \backslash U_{0}, \Psi_{m}$ is the identity while on the region $U_{0} \backslash W_{-1}$ it is a reflection with respect to height $h\left(\partial_{\circ} W_{0}\right)-\delta / 2=h\left(\partial_{\bullet} U_{0}\right)$.

Proposition 5.3. $\Psi_{m}$ is a one-to-one mapping from $\mathrm{QP}_{m}$ to $\mathrm{QP}_{\mathbf{0}}$.

Proof. Write $t:=\Psi_{m}(h)$. We need to show that $t$ is periodic in $n e_{i}$ for every $1 \leq i \leq d$, that it is a height function, and that $\Psi_{m}$ is one-to-one.

$t$ is periodic

First we show that for every $\Delta^{\prime} \in n \mathbb{Z}^{d}, a \in \mathbb{Z}$ such that $W_{0}+\Delta^{\prime}=W_{a}$, we have

$$
h(v)=h\left(v+\Delta^{\prime}-a \Delta\right) \text { for all } v \in \mathbb{Z}^{d} .
$$

By quasi-periodicity, for all $v \in \mathbb{Z}^{d}$, we have $h\left(v+\Delta^{\prime}-a \Delta\right)=h(v)+\left(h\left(\Delta^{\prime}-a \Delta\right)-h(\mathbf{0})\right)$. Hence it suffices to prove (5.12) for a single $v \in \mathbb{Z}^{d}$. Next, note that since $W_{0}=W_{a}-\Delta^{\prime}=W_{0}+a \Delta-\Delta^{\prime}$ we have that if $v \in \partial_{\bullet} W_{0}$, then $v+\Delta^{\prime}-a \Delta$ is also a member of $\partial_{\bullet} W_{0}$, implying, by the definition of $W_{0}$ that $h(v)=h\left(v+\Delta^{\prime}-a \Delta\right)$. This establishes (5.12).

Now, let $1 \leq j \leq d$, and suppose that $o_{W_{0}}\left(W_{0}+n e_{j}\right)=a \in \mathbb{Z}$ where $o_{W_{0}}$ is the order function of $W_{0}$ given by Theorem 3.4. Observe that $W_{0}+n e_{j}=W_{a}$. Note that if $v \in W_{i} \backslash U_{i}$ then, by Proposition 5.2, $v+n e_{j} \in W_{i+a} \backslash U_{i+a}$. Thus, using (5.12), if $v \in W_{i} \backslash U_{i}$ then

$$
t(v)=h(v-i \Delta)=h\left(v+n e_{j}-(i+a) \Delta\right)=t\left(v+n e_{j}\right) .
$$

Similarly, if $v \in U_{i+1} \backslash W_{i}$ then, using Proposition 5.2, we have

$$
t(v)=2 h\left(\partial_{\circ} W_{0}\right)-h(v-i \Delta)=2 h\left(\partial_{\circ} W_{0}\right)-h\left(v+n e_{j}-(i+a) \Delta\right)=t\left(v+n e_{j}\right) .
$$

$t$ is an $\mathrm{HHF}$

We claim that $t \in \operatorname{Hom}\left(\mathbb{Z}^{d}\right)$, i.e., that the values which $t$ assigns to adjacent vertices differ by exactly 1 . Let $u, v$ be 
adjacent vertices in $\mathbb{Z}^{d}$. We need to show that

$$
|t(u)-t(v)|=1 .
$$

Since $t$ is $\Delta$-periodic, for every vertex $w \in \mathbb{Z}^{d}$ there exists $j \in \mathbb{Z}$ such that $w+j \Delta \in U_{1} \backslash U_{0}$ and $t(w)=t(w+j \Delta)$. We may therefore assume WLOG $u \in U_{1} \backslash U_{0}$, and $v \in U_{1}$. We consider three cases separately.

First, if both $u, v \in U_{1} \backslash W_{0}$ or both $u, v \in W_{0} \backslash U_{0}$ then (5.13) follows directly from the definition of $\Psi_{m}$.

Second, note that

$$
t\left(\partial_{\circ} W_{0}\right)-t\left(\partial_{\bullet} W_{0}\right)=2 h\left(\partial_{\circ} W_{0}\right)-h\left(\partial_{\circ} W_{0}\right)-h\left(\partial_{\bullet} W_{0}\right)=h\left(\partial_{\circ} W_{0}\right)-h\left(\partial_{\bullet} W_{0}\right)=1 .
$$

Hence (5.13) holds if either $u \in \partial_{\circ} W_{0}$ and $v \in \partial_{\bullet} W_{0}$ or vice versa.

Third,

$$
t\left(\partial_{\circ} U_{0}\right)-t\left(\partial_{\bullet} U_{0}\right)=h\left(\partial_{\circ} U_{0}\right)-\left(2 h\left(\partial_{\circ} W_{0}\right)-h\left(\partial_{\bullet} U_{0}\right)-\delta\right),
$$

and plugging the relation $h\left(\partial_{\circ} W_{0}\right)=h\left(\partial_{\bullet} U_{0}\right)+\delta / 2$ from (5.3) yields

$$
t\left(\partial_{\circ} U_{0}\right)-t\left(\partial_{\bullet} U_{0}\right)=h\left(\partial_{\circ} U_{0}\right)-h\left(\partial_{\bullet} U_{0}\right)=1 .
$$

Thus (5.13) holds if $u \in \partial_{\circ} U_{0}$ and $v \in \partial_{\bullet} U_{0}$.

$\Psi_{m}$ is one-to-one

To show that $\Psi_{m}$ is one-to-one, we explain how to construct an inverse for it. Suppose that we are able to recover $U_{0}, W_{0}, \Delta$ and $\delta$ from $t$ and $m$. Then we may define $U_{i}=U_{0}+i \Delta, W_{i}=W_{0}+i \Delta$ and the mapping

$$
\Psi_{m}^{-1}(t)(v):= \begin{cases}t(v)+i \delta, & v \in W_{i} \backslash U_{i} \text { for some } i \in \mathbb{Z}, \\ 2 t\left(\partial_{\circ} W_{0}\right)-t(v)+i \delta, & v \in U_{i+1} \backslash W_{i} \text { for some } i \in \mathbb{Z} .\end{cases}
$$

It is simple to check that this $\Psi_{m}^{-1}$ is indeed an inverse to $\Psi_{m}$. It is therefore sufficient to show that $U_{0}, W_{0}, \Delta$ and $\delta$ may be recovered from $t$ and $m$.

We begin by recovering $W_{0}$. To do this we follow the lines of the proof of proposition 5.1. Write $\mathcal{W}_{t}$ for the set of type 0 sublevel components of $t$ which contain $\mathbf{0}$ and do not contain $n e_{1}$. Again we recall (4.4) and observe that $\mathcal{W}_{t} \subseteq \mathcal{L}_{\left(0, n e_{1}\right)}$, where $\mathcal{L}$ is defined with respect to $t$. Thus, by Proposition $4.6, \mathcal{W}_{t}$ is ordered by inclusion and finite. We now argue that $\mathcal{W}_{t}$ is a non-empty set whose minimal element is $W_{0}$.

The definition (5.11) of $\Psi_{m}$ and the relation $h\left(\partial_{\circ} W_{0}\right)=h\left(\partial_{\bullet} U_{0}\right)+\delta / 2$ from (5.3) imply that

$$
t(x)=h(x) \text { for } x \in W_{0}^{+} \backslash U_{0}^{-} .
$$

We can therefore apply Proposition 4.14 with $h_{1}=h, h_{2}=t, A=W_{0}$ and $B=U_{0}$ to get that

$$
W_{0} \in \mathcal{W}_{t}
$$

Applying the same proposition with $A=V_{0}$ yields that

$V_{0}$ is a sublevel component of $t$.

Let us write $W_{t}$ for the minimal element of $\mathcal{W}_{t}$. Since $W_{0} \in \mathcal{W}_{t}$ we conclude that

$$
W_{t} \subseteq W_{0} .
$$

To obtain the opposite inclusion we now show that $W_{t}$ is also a sublevel component of $h$. Observe that since $W_{t}$ is of type 0 , and since $\mathbf{0} \in W_{t}$ and $n e_{1} \notin W_{t}$ we have by Theorem 3.4 that $W_{t}-n e_{1} \subsetneq W_{t}$. We deduce that $-n e_{1} \in$ $W_{t} \cap V_{0}$. In addition, our definitions imply that $n e_{1} \in\left(W_{t}\right)^{c} \cap\left(V_{0}\right)^{c}$ and $\mathbf{0} \in\left(W_{t} \backslash V_{0}\right)$. By Theorem 3.2, using 
that distinct sublevel components of $t$ are boundary disjoint by Proposition 4.3, we deduce that $V_{0} \subsetneq W_{t}$. Applying Proposition 4.14 with $h_{1}=t, h_{2}=h, A=W_{t}$ and $B=V_{0}$, using (5.14) and (5.17) to check the condition (4.8), we get that $W_{t}$ is a sublevel component of $h$. Together with (5.17), the minimality of $W_{0}$ now implies that $W_{t}=W_{0}$, allowing the recovery of $W_{0}$ from $t$. After recovering $W_{0}$, we can recover $\Delta$ and $\delta$ using the fact that $\Delta$ is a minimal translation of $W_{0}$ chosen in a prescribed manner and the fact that by Corollary 4.9 we have $\delta \cdot o_{W_{0}}\left(W_{0}+n e_{1}\right)=m_{1}$, where $o_{W_{0}}$ is the order function on translations of $W_{0}$, given by Theorem 3.4.

All that remains is to recover $U_{0}$. Following again the lines of the proof of Proposition 5.1, we write $\mathcal{U}_{t}$ for the set containing all $U^{\prime}$ such that $\left(U^{\prime}\right)^{c}$ is a type 0 superlevel component of $t$ and $t\left(\partial_{\bullet} U^{\prime}\right)=t\left(\partial_{\circ} W_{0}\right)-\delta / 2, W_{0}-\Delta \subseteq U^{\prime} \subseteq$ $W_{0}, \mathbf{0} \notin U^{\prime}$ and $-n e_{1} \in U^{\prime}$. Recalling Definition 4.12 of superlevel sets we again use Proposition 4.6 to deduce that the set of superlevel components containing $\mathbf{0}$ and not containing $-n e_{1}$ is finite and ordered by inclusion, implying that $\mathcal{U}_{t}$ is also finite and ordered by inclusion. We now use (5.14) and Proposition 4.14, with $h_{1}=-h, h_{2}=-t$, $A=\left(U_{0}\right)^{c}$ and $B=\left(W_{0}\right)^{c}$, to get that $U_{0}^{c}$ is a superlevel component of $t$ (again, using Definition 4.12 of superlevel components). It follows from (5.14) that $U_{0} \in \mathcal{U}_{t}$. Write $U_{t}$ for the maximal element of $\mathcal{U}_{t}$, i.e., the complement of the minimal element amongst complements of elements in $\mathcal{U}_{t}$. Since $U_{0} \in \mathcal{U}_{t}$ we conclude that

$$
U_{t}^{c} \subseteq U_{0}^{c}
$$

Recall that, by the definition of $\mathcal{U}_{t}$, we have $\left(W_{0}\right)^{c} \subsetneq\left(U_{t}\right)^{c}$ and that, by (5.15), $W_{0}$ is also a sublevel component of $t$. Applying Proposition 4.14 to $h_{1}=-t, h_{2}=-h, A=\left(U_{t}\right)^{c}$ and $B=\left(W_{0}\right)^{c}$, using (5.14) and (5.18) to check the condition (4.8), we get that $U_{t}^{c}$ is also a superlevel component of $h$. We also have $h\left(\partial_{\bullet} U_{t}\right)=h\left(\partial_{\circ} W_{0}\right)-\delta / 2$ by (5.14). Thus, together with (5.18), the minimality of $U_{0}^{c}$ now implies that $U_{0}=U_{t}$. As $W_{0}, U_{0}, \Delta$ and $\delta$ can be recovered from $t$ and $m$, we deduce that $\Psi_{m}$ is one-to-one.

\subsection{Proof of Theorem 2.3}

In this section we prove Theorem 2.3 using a bound on the probability for a uniformly chosen HHF on the torus to have a sublevel component with long boundary. Here, for the first time, we use sublevel components on $\mathbb{T}_{n}^{d}$ (defined in Section 4). To clarify our proof we will always denote HHFs in Hom( $\left.\mathbb{T}_{n}^{d}\right)$ by $r$, HHFs in $\mathrm{QP}_{\mathbf{0}}$ by $t$ and HHFs in $\mathrm{QP}_{m}$, for arbitrary $m=\left(m_{1}, \ldots, m_{d}\right)$ with $m_{1}>0$, by $h$.

Recall that for $u \in \mathbb{T}_{n}^{d}$ we denoted by $\operatorname{Hom}\left(\mathbb{T}_{n}^{d}, u\right)$ the set of all homomorphism height functions on $\mathbb{T}_{n}^{d}$ which are zero at $u$. We use the following theorem of [18] to derive the estimates of Theorem 2.3.

Theorem 5.4 ([18, special case of Theorem 2.8]). There exist $c>0$ and $d_{0}$ such that in all dimensions $d \geq d_{0}$, for all even $n$, all $u, v \in \mathbb{T}_{n}^{d}$ and all $L \geq 1$, if $h$ is uniformly sampled from $\operatorname{Hom}\left(\mathbb{T}_{n}^{d}, u\right)$ then

$$
\mathbb{P}\left(\left|\partial \operatorname{LC}_{h}^{0+}(u, v)\right| \geq L\right) \leq d \exp \left(-\frac{c L}{d \log ^{2} d}\right),
$$

where we mean that $\mathrm{LC}_{h}^{0+}(u, v)=\varnothing$ if $h(v) \leq 0$.

We adapt Theorem 5.4 to our setting through the following corollary.

Corollary 5.5. There exist $c>0$ and $d_{0}$ such that in all dimensions $d \geq d_{0}$, for all even $n$ and all $L \geq 1$, denoting

$$
A:=\left\{r \in \operatorname{Hom}\left(\mathbb{T}_{n}^{d}\right): \text { there exists a sublevel component } A \text { such that }|\partial A| \geq L\right\},
$$

the following holds,

$$
\frac{|A|}{\left|\operatorname{Hom}\left(\mathbb{T}_{n}^{d}\right)\right|} \leq 2 d^{2} n^{d} \exp \left(-\frac{c L}{d \log ^{2} d}\right) .
$$


Proof. Fix $L \geq 1$ and let $B:=\left\{r \in \operatorname{Hom}\left(\mathbb{T}_{n}^{d}\right): \exists v \in \mathbb{T}_{n}^{d}, v \sim \mathbf{0}\right.$, s.t. $\left.\left|\partial \operatorname{LC}_{r}^{+}(\mathbf{0}, v)\right| \geq L\right\}$. By Theorem 5.4 with $u=\mathbf{0}$, and using a union bound on all $v \sim \mathbf{0}$, we have

$$
|B| \leq 2 d \cdot d \exp \left(-\frac{c L}{d \log ^{2} d}\right)\left|\operatorname{Hom}\left(\mathbb{T}_{n}^{d}\right)\right| \text { for all } d \text { greater then some fixed } d_{0} .
$$

Now, for every $w \in \mathbb{T}_{n}^{d}$ define the mapping $\eta_{w}: \operatorname{Hom}\left(\mathbb{T}_{n}^{d}\right) \rightarrow \operatorname{Hom}\left(\mathbb{T}_{n}^{d}\right)$ by

$$
\eta_{w}(r)(v):=r(v+w)-r(w) .
$$

It is not difficult to check that this mapping is well defined and is a bijection. Moreover, for every $r \in A$ there exists a $w \in \mathbb{T}_{n}^{d}$ such that $\eta_{w}(r) \in B$. The corollary follows.

In order to apply Corollary 5.5, we must show that HHFs in the image of $\Psi_{m}$, when projected to the torus, contain a sublevel component with a long boundary. We proceed in two steps. First, we claim that the projection of the boundary of the set $V_{0}$ from Proposition 5.1 is contained in the boundary of a sublevel component of the projection of $\Psi_{m}(h)$. Then we claim that this boundary is long. Recall that $\pi$ was defined in Section 2.1 to be the natural projection from $\mathbb{Z}^{d}$ to $\mathbb{T}_{n}^{d}$. Here we use also the natural extension of $\pi$ to edges of $\mathbb{Z}^{d}$.

Lemma 5.6. Let $h \in \mathrm{QP}_{m}$ for $m \in 6 \mathbb{Z}^{d}$ satisfying $m_{1}>0$. Let $r=\pi \circ \Psi_{m}(h)$ and $V_{0}$ be as in Proposition 5.1. There exists a sublevel component $R$ of $r$ such that $\pi\left(\partial V_{0}\right) \subseteq \partial R$.

We delay the proof of this lemma to Section 5.3.

At last we are ready to prove the theorem.

Proof of Theorem 2.3. Let $m \in 6 \mathbb{Z}^{d} \backslash\{\boldsymbol{0}\}$. Using an appropriate rotation we may assume without loss of generality that $m_{1}>0$. Fixing $h \in \mathrm{QP}_{m}$ and applying Lemma 5.6 and Lemma 3.8, we obtain the existence of a sublevel component $R$ of $\pi \circ \Psi_{m}(h)$ such that $|\partial R| \geq n^{d-1}$. Thus

$$
\pi\left(\Psi_{m}\left(\mathrm{QP}_{m}\right)\right) \subset\left\{r \in \operatorname{Hom}\left(\mathbb{T}_{n}^{d}\right): \text { there exists a sublevel component } A \text { of } r \text { such that }|\partial A| \geq n^{d-1}\right\} .
$$

Recall that $\pi$ is a bijection from $\mathrm{QP}_{\mathbf{0}}$ to $\operatorname{Hom}\left(\mathbb{T}_{n}^{d}\right)$. Thus, applying Corollary 5.5, we get that for large enough $d$,

$$
\left|\Psi_{m}\left(\mathrm{QP}_{m}\right)\right| \leq 2 d^{2} n^{d} \exp \left(-\frac{c n^{d-1}}{d \log ^{2} d}\right)\left|\operatorname{Hom}\left(\mathbb{T}_{n}^{d}\right)\right| \leq \exp \left(-\frac{c^{\prime} n^{d-1}}{d \log ^{2} d}\right)\left|\mathrm{QP}_{\mathbf{0}}\right|
$$

for some $c, c^{\prime}>0$. Thus, since $\Psi_{m}$ is one-to-one, the theorem follows.

\subsection{Projecting type 0 sublevel components to the torus}

In this section we prove Lemma 5.6 connecting sublevel components on $\mathrm{QP}_{\mathbf{0}}$ with those on $\mathrm{Hom}\left(\mathbb{T}_{n}^{d}\right)$. While the relation between sublevel components of HHFs on the integer lattice and those of HHFs on the torus is non-trivial, the relation between sublevel sets of the two spaces is much simpler. In particular,

$$
\pi\left(\operatorname{LC}_{t}^{+}(u)\right)=\operatorname{LC}_{\pi(t)}^{+}(\pi(u)) \quad \text { for all } t \in \mathrm{QP}_{\mathbf{0}} \text { and } u \in \mathbb{Z}^{d} .
$$

This can be easily verified from the definition of sublevel sets.

Next, we prove a proposition relating the boundaries of sublevel components on $\mathbb{Z}^{d}$ to those of sublevel components on $\mathbb{T}_{n}^{d}$. We then show that this proposition applies to the set $V_{0}$ from Proposition 5.1, and use this fact to prove Lemma 5.6. We remind the reader that $A^{+}$and $A^{++}$were introduced in Section 2.1.

Proposition 5.7. Let $t \in \mathrm{QP}_{0}$ and $r=\pi(t) \in \operatorname{Hom}\left(\mathbb{T}_{n}^{d}\right)$. Suppose $V:=\operatorname{LC}_{t}^{+}(u, v)$ for adjacent vertices $u, v \in \mathbb{Z}^{d}$ satisfying $t(v)=t(u)+1$. If

$$
\pi\left(V^{++} \backslash V\right) \cap \pi\left(\mathrm{LC}_{t}^{+}(u)\right)=\varnothing
$$


then

$$
\pi(\partial V) \subseteq \partial \mathrm{LC}_{r}^{+}(\pi(u), \pi(v)) .
$$

Proof. Let $R:=\mathrm{LC}_{r}^{+}(\pi(u), \pi(v))$. We first note that (5.21) follows from the following two claims,

$$
\begin{aligned}
& \pi\left(\partial_{\bullet} V\right) \subseteq R, \\
& \pi\left(\partial_{\diamond} V\right) \subseteq R^{c} .
\end{aligned}
$$

We begin by showing (5.22). Indeed, we have:

$$
\pi\left(\partial_{\bullet} V\right) \subseteq \pi\left(\operatorname{LC}_{t}^{+}(u)\right)=\mathrm{LC}_{r}^{+}(\pi(u)) \subseteq R,
$$

where the equality follows from (5.19), and the two containment relations follow from Proposition 4.1.

Next we show (5.23). By Proposition 3.1, using the fact that $V$ is co-connected by Proposition 4.1, we get that $\pi\left(V^{++} \backslash V\right)$ is a connected set which contains $\pi(v)$ (recall that $\left.u \sim v\right)$. By (5.19) and (5.20), $\pi\left(V^{++} \backslash V\right)$ is disjoint from $\operatorname{LC}_{r}^{+}(\pi(u))$. By the definition of sublevel component this implies that $\pi\left(V^{++} \backslash V\right) \subseteq R^{c}$. Since $\pi\left(\partial_{\circ} V\right) \subseteq$ $\pi\left(V^{++} \backslash V\right)$, we deduce (5.23).

At last, we prove Lemma 5.6. Let $h \in \mathrm{QP}_{m}$ for $m \in 6 \mathbb{Z}^{d}$ satisfying $m_{1}>\mathbf{0}$. Let $U=U_{0}, V=V_{0}, W=W_{0}$ and $\Delta$ be as in Proposition 5.1. Let also $t:=\Psi_{m}(h)$ and $r:=\pi(t)$. Our goal is to show that $V$ satisfies the conditions of Proposition 5.7, from which Lemma 5.6 will follow.

Write $\mathcal{T}$ for the set of type 0 sublevel components $T^{\prime}$ satisfying $h\left(\partial_{\bullet} T^{\prime}\right)=h\left(\partial_{\circ} W\right)-\delta+1$ and $W-\Delta \subsetneq T^{\prime} \subsetneq V$. Recall that $W-\Delta \subsetneq V$ by (5.4), $h\left(\partial_{\bullet}(W-\Delta)\right)=h\left(\partial_{\bullet} W\right)-\delta, h\left(\partial_{\bullet} V\right)=h\left(\partial_{\bullet} W\right)-1$ by (5.2) and that $\delta \geq 6$ by (5.7). Hence, by Lemma 4.13, we conclude that $\mathcal{T}$ is non-empty. Write $T$ for the minimal element of $\mathcal{T}$.

Let us show that $T \subseteq U$. By Lemma 4.13 applied to $T \subsetneq V$, using that $h\left(\partial_{\bullet} T\right)=h\left(\partial_{\circ} W\right)-\delta+1$ and $h\left(\partial_{\bullet} V\right)=$ $h\left(\partial_{\bullet} W\right)-1$, there exists a $U^{\prime}$ satisfying that $\left(U^{\prime}\right)^{c}$ is a type 0 superlevel component such that $h\left(\partial_{\bullet} U^{\prime}\right)=h\left(\partial_{\circ} W\right)-\delta / 2$ and $T \subseteq U^{\prime} \subsetneq V$. Next, observe that $\mathbf{0} \notin U^{\prime}$, since $0 \notin V$ by (5.2), and that $-n e_{1} \in U^{\prime}$, since $-n e_{1} \in W-\Delta \subsetneq T$ by (5.8). Thus, (5.4) and the definition of $U$ (in particular, the fact that $U^{c}$ is minimal), imply that $U^{\prime} \subseteq U$. We conclude that

$$
W-\Delta \subsetneq T \subseteq U
$$

Next, the definition (5.11) of $\Psi_{m},(5.24)$ and the definition of $T$ imply that

$$
t\left(\partial_{\bullet} T\right)=2 h\left(\partial_{\circ} W\right)-h\left(\partial_{\bullet} T\right)-\delta=h\left(\partial_{\circ} W\right)-1 .
$$

Now, since $U \subsetneq V \subsetneq W$ by (5.4), the definition of $\Psi_{m}$ implies that

$$
h\left(\partial_{\circ} V\right)=t\left(\partial_{\circ} V\right) .
$$

Thus, by (5.2),

$$
t\left(\partial_{\bullet} T\right)=t\left(\partial_{\circ} V\right) .
$$

We now check that $V$ satisfies the conditions of Proposition 5.7. Recall that by (5.16), $V$ is a sublevel component of $t$. Let $u \in \partial_{\bullet} V, v \in \partial_{\circ} V$ be two adjacent vertices. By Corollary 4.4 we have $V=\operatorname{LC}_{t}^{+}(u, v)$. Observe that the condition (5.20) is equivalent to

$$
\left(\left(V^{++} \backslash V\right)+z\right) \cap \operatorname{LC}_{t}^{+}(u)=\varnothing \quad \text { for all } z \in n \mathbb{Z}^{d} .
$$

Since $\left(V^{++} \backslash V\right)+z=\left(V^{++}+z\right) \backslash(V+z)$ and since $V$ is of type 0 having, by Proposition 5.2, $\Delta$ as a minimal translation, this is equivalent to

$$
\left(\left(V^{++}+k \Delta\right) \backslash(V+k \Delta)\right) \cap \mathrm{LC}_{t}^{+}(u)=\varnothing \quad \text { for all } k \in \mathbb{Z} .
$$


We note that $T \subsetneq V$ by the definition of $T$. It follows from (5.25) that the set $S:=V \backslash T$ satisfies $t(s)=t\left(\partial_{\circ} V\right)$ for all $s \in \partial_{\circ} S$. As $u \notin T$, this implies that $\operatorname{LC}_{t}^{+}(u) \subseteq S$. Thus, to check condition (5.26) it suffices to show that

$$
\left(\left(V^{++}+k \Delta\right) \backslash(V+k \Delta)\right) \cap S=\varnothing \quad \text { for all } k \in \mathbb{Z},
$$

which, since $S=V \backslash T$, is itself implied by

$$
\begin{aligned}
& V^{++}+k \Delta \subseteq T \quad \text { for all } k \leq-1, \\
& V+k \Delta \supseteq V \quad \text { for all } k \geq 0 .
\end{aligned}
$$

Since $\Delta$ is a minimal translation for $V$, the second part of (5.27) follows trivially and it suffices to check the first part for $k=-1$. Finally, the condition that $\left(V^{++}-\Delta\right) \subseteq T$ follows from the fact that $V-\Delta, W-\Delta$ and $T$ are boundary disjoint and satisfy $V-\Delta \subsetneq W-\Delta \subsetneq T$. This is a consequence of (5.4), Proposition 4.3 and the definition of $T$. Thus the condition of Proposition 5.7 is satisfied. Lemma 5.6 follows from (5.21).

\section{Steep slopes are extremely unlikely}

In this section we detail how to modify the proof of Theorem 2.3 to prove Theorem 2.4 .

A main ingredient in the proof of the theorem is the following proposition which is a consequence of the results of [18].

Proposition 6.1 (Enhanced version of Corollary 5.5). There exist $c>0$ and $d_{0}$ such that in all dimensions $d \geq d_{0}$, for all even $n$, all integer $k \geq 1$ and $L \geq n^{d-1}$, denoting

$$
A:=\left\{r \in \operatorname{Hom}\left(\mathbb{T}_{n}^{d}\right): \exists u, v \in \mathbb{T}_{n}^{d} \text { s.t. } r(v) \geq r(u)+k \text { and } \sum_{j=0}^{k-1}\left|\partial \operatorname{LC}_{r}^{(r(u)+j)+}(u, v)\right| \geq L\right\},
$$

the following holds,

$$
\frac{|A|}{\left|\operatorname{Hom}\left(\mathbb{T}_{n}^{d}\right)\right|} \leq \exp \left(-\frac{c L}{d \log ^{2} d}\right)
$$

We make the assumption that $L \geq n^{d-1}$ in order to simplify the proof of the proposition and as it suffices for our purposes in this section but we remark that similar estimates may be established for all $L \geq 1$ using additional arguments (e.g., the isoperimetric estimates of [18, Theorem 5.1]).

Proof of Proposition 6.1. Define

$$
B:=\left\{r \in \operatorname{Hom}\left(\mathbb{T}_{n}^{d}\right): \exists v \in \mathbb{T}_{n}^{d} \text { s.t. } r(v) \geq k \text { and } \sum_{j=0}^{k-1}\left|\partial \operatorname{LC}_{r}^{j+}(\mathbf{0}, v)\right| \geq L\right\}
$$

Using a similar argument as in the proof of Corollary 5.5 (defining the mapping $\eta_{w}$ ) it suffices to show that

$$
\frac{|B|}{\left|\operatorname{Hom}\left(\mathbb{T}_{n}^{d}\right)\right|} \leq n^{-d} \exp \left(-\frac{c L}{d \log ^{2} d}\right) .
$$

Equation (72) in [18, Proof of Proposition 5.15] implies that for each $\left(L_{j}\right), 0 \leq j<k$, with $L_{j} \geq 1$ we have

$$
\frac{\mid\left\{r \in \operatorname{Hom}\left(\mathbb{T}_{n}^{d}\right): \exists v \in \mathbb{T}_{n}^{d} \text { s.t. }\left|\partial \operatorname{LC}_{r}^{j+}(\mathbf{0}, v)\right|=L_{j} \text { for all } 0 \leq j<k\right\} \mid}{\left|\operatorname{Hom}\left(\mathbb{T}_{n}^{d}\right)\right|} \leq n^{d} d^{k} \exp \left(-\frac{c^{\prime} \sum_{j=0}^{k-1} L_{j}}{d \log ^{2} d}\right),
$$


for some $c^{\prime}>0$. We deduce that

$$
\begin{aligned}
\frac{|B|}{\left|\operatorname{Hom}\left(\mathbb{T}_{n}^{d}\right)\right|} & \leq \sum_{\bar{L}=L}^{\infty} n^{d} d^{k} \exp \left(-\frac{c^{\prime} \bar{L}}{d \log ^{2} d}\right)\left|\left\{\left(L_{0}, \ldots, L_{k-1}\right): L_{j} \geq 1, L_{0}+\cdots+L_{k-1}=\bar{L}\right\}\right| \\
& =n^{d} d^{k} \sum_{\bar{L}=L}^{\infty}\left(\begin{array}{l}
\bar{L}-1 \\
k-1
\end{array}\right) \exp \left(-\frac{c^{\prime} \bar{L}}{d \log ^{2} d}\right) \leq n^{d} d^{k} \sum_{\bar{L}=L}^{\infty} \bar{L}^{k} \exp \left(-\frac{c^{\prime} \bar{L}}{d \log ^{2} d}\right) .
\end{aligned}
$$

Next observe that if $B$ is non-empty then $k$ is at most the diameter of $\mathbb{T}_{n}^{d}$. Thus we assume without loss of generality that $k \leq d n$. Recalling also our assumption that $L \geq n^{d-1}$, we see that the ratio of consecutive terms in the last sum equals

$$
\left(1+\frac{1}{\bar{L}}\right)^{k} \exp \left(-\frac{c^{\prime}}{d \log ^{2} d}\right) \leq \exp \left(-\frac{c^{\prime}}{d \log ^{2} d}+\frac{k}{\bar{L}}\right) \leq \exp \left(-\frac{c^{\prime}}{d \log ^{2} d}+\frac{d}{n^{d-2}}\right) \leq \exp \left(-\frac{c^{\prime}}{2 d \log ^{2} d}\right)
$$

for large enough $d$. Thus we conclude that

$$
\begin{aligned}
\frac{|B|}{\left|\operatorname{Hom}\left(\mathbb{T}_{n}^{d}\right)\right|} & \leq n^{d}(d L)^{k} \exp \left(-\frac{c^{\prime} L}{d \log ^{2} d}\right) \sum_{\bar{L}=L}^{\infty} \exp \left(-\frac{c^{\prime}(\bar{L}-L)}{2 d \log ^{2} d}\right) \\
& \leq n^{d}(d L)^{k} d^{2} \exp \left(-\frac{c^{\prime} L}{d \log ^{2} d}\right) \leq n^{-d} \exp \left(-\frac{c^{\prime} L}{2 d \log ^{2} d}\right)
\end{aligned}
$$

for large enough $d$, where in the last step we used again that $k \leq d n$ and $L \geq n^{d-1}$.

We begin by formulating various properties of functions in $\mathrm{QP}_{m}$ which are needed for the proof of Theorem 2.4 . Fix $m \in 6 \mathbb{Z}^{d} \backslash\{\boldsymbol{0}\}$. Assume that $\mathrm{QP}_{m} \neq \varnothing$ as the theorem is trivial otherwise. Assume also, without loss of generality, that the first coordinate of $m$ is positive and is the largest in absolute value among all coordinates, and write

$$
\sigma:=\frac{m_{1}}{6}=\frac{h\left(n e_{1}\right)}{6} .
$$

Fix $h \in \mathrm{QP}_{m}$ and recall our definition of $W_{0}, \Delta, \delta$ and $\Psi_{m}$ from Section 5.1. We write

$$
p:=\frac{\delta}{6} \quad \text { and } \quad \ell:=\frac{\sigma}{p}=\frac{h\left(n e_{1}\right)}{\delta} .
$$

We remark that it is possible to show that $\delta, p$ and $\ell$ depend only on $m$, but since this fact will not be of use to us, we do not prove it. Observe that $\sigma$ is a positive integer as $m \in 6 \mathbb{Z}^{d}$ and $m_{1}>0$. In addition, by (5.5), (5.6), (5.7) and the argument in the paragraph there, we have that $p$ and $\ell$ are also positive integers and that

$$
W_{0}+n e_{1}=W_{0}+\ell \Delta .
$$

We wish to find a single pair of vertices separated by $p$ sublevel components of $\pi \circ \Psi_{m}(h)$, each with boundary size at least $\ell n^{d-1}$. We remark that more components may be found, at least as many as $\frac{\delta}{2}-2$, but this is not required for our results. We proceed by defining additional type 0 sublevel components of $h$, whose roles are similar to the role of $V_{0}$ in the proof of Theorem 2.3 (Section 5).

Define $V_{0}^{p-1}$, as the maximal type 0 sublevel component of $h$ satisfying

$$
h\left(\partial_{\bullet} V_{0}^{p-1}\right)=h\left(\partial_{\bullet} W_{0}\right)-3 p+2, \quad W_{0}-\Delta \subseteq V_{0}^{p-1} \subseteq W_{0}, \quad \mathbf{0} \notin V_{0}^{p-1} \text { and } \quad-n e_{1} \in V_{0}^{p-1} .
$$

Further define $V_{0}^{i}, 0 \leq i<p-1$, as the minimal type 0 sublevel component of $h$ satisfying

$$
h\left(\partial_{\bullet} V_{0}^{i}\right)=h\left(\partial_{\bullet} W_{0}\right)-3 i-1, \quad V_{0}^{p-1} \subseteq V_{0}^{i} \subseteq W_{0} .
$$


We recall the set $U_{0}$ defined in Section 5.1. The following proposition is a generalization of the part of Proposition 5.1 which pertains to $V_{0}$.

Proposition 6.2. $\left\{V_{0}^{i}\right\}_{i=0}^{p-1}$ are well defined, and satisfy

$$
U_{0} \subsetneq V_{0}^{p-1} \subsetneq \cdots \subsetneq V_{0}^{0} \subsetneq W_{0} .
$$

Proof. Following the proof of Proposition 5.1 we write $\mathcal{V}^{p-1}$ for the set of type 0 sublevel components $V^{\prime}$ satisfying $h\left(\partial_{\bullet} V^{\prime}\right)=h\left(\partial_{\bullet} W_{0}\right)-3 p+2, W_{0}-\Delta \subsetneq V^{\prime} \subsetneq W_{0}, \mathbf{0} \notin V^{\prime}$ and $-n e_{1} \in V^{\prime}$. We observe that $\mathcal{V}^{p-1} \subseteq \mathcal{L}_{\left(-n e_{1}, 0\right)}$, and thus, by Proposition 4.6, $\mathcal{V}^{p-1}$ is ordered by inclusion and finite. Moreover, applying the first part of Lemma 4.13 with $h, W_{0}-\Delta$ and $W_{0}$ we obtain the existence of a type 0 sublevel component $V_{p-1}^{\prime}$ which satisfies $h\left(\partial_{\bullet} V_{p-1}^{\prime}\right)=$ $h\left(\partial_{\bullet} W_{0}\right)-3 p-2$ and $W_{0}-\Delta \subsetneq V_{p-1}^{\prime} \subsetneq W_{0}$. By the minimality of $W_{0}$ we have $\mathbf{0} \notin V_{p-1}^{\prime}$. As $-n e_{1} \in W_{0}-\Delta$ by (5.8), we have $-n e_{1} \in V_{p-1}^{\prime}$ and hence $V_{p-1}^{\prime} \in \mathcal{V}^{p-1}$ and $\mathcal{V}^{p-1}$ is not empty. We deduce that $V_{0}^{p-1}$ is well defined.

For $0 \leq i<p-1$ we now define $\mathcal{V}^{i}$ as the set of type 0 sublevel components $V^{\prime}$ satisfying $h\left(\partial_{\bullet} V^{\prime}\right)=h\left(\partial_{\bullet} W_{0}\right)-$ $3 i-1, V_{0}^{p-1} \subsetneq V^{\prime} \subsetneq W_{0}$. Using the same arguments as above we observe that $\mathcal{V}^{i}$ is ordered by inclusion and finite. Applying once again the first part of Lemma 4.13, now with $h, V_{0}^{p-1}$ and $W_{0}$ we obtain the existence of a a type 0 sublevel component $V_{i}^{\prime}$ which satisfies $h\left(\partial_{\bullet} V_{i}^{\prime}\right)=h\left(\partial_{\bullet} W_{0}\right)-3 i-1$ and $V_{0}^{p-1} \subsetneq V_{i}^{\prime} \subsetneq W_{0}$. Thus $\mathcal{V}^{i}$ is not empty and $V_{0}^{i}$ is well defined.

We proceed to show the inclusion relations (6.3). We have $V_{0}^{0} \subsetneq W_{0}$ and $V_{0}^{p-1} \subsetneq V_{0}^{p-2}$ (when $p>1$ ) by the definition of $V_{0}^{0}$ and $V_{0}^{p-2}$. Next, for each $0<i<p-1$ the first part of Lemma 4.13 applied with $h, V_{0}^{p-1}$ and $V_{0}^{i-1}$ shows that there exists an element $V_{i}^{\prime \prime} \in \mathcal{V}^{i}$ satisfying $V_{0}^{p-1} \subsetneq V_{i}^{\prime \prime} \subsetneq V_{0}^{i-1}$, whence the inclusion $V_{0}^{i} \subsetneq V_{0}^{i-1}$ follows from the minimality of $V_{0}^{i}$. It remains to show that $U_{0} \subsetneq V_{0}^{p-1}$ and this is done next by exhibiting an element of $\mathcal{V}^{p-1}$ which strictly contains $U_{0}$.

To do so we repeat the arguments in the proof of Proposition 5.1 concerning the fact that $U_{0} \subsetneq V_{0}$. Let $u \in \partial_{\bullet} U_{0}$, $w \in \partial_{\circ} W_{0}$ and write $V_{p-1}^{\prime \prime}=\mathrm{LC}_{h}^{\left(h\left(\partial_{\bullet} W_{0}\right)-3 p+2\right)+}(u, w)$. Our goal is to show that $V_{p-1}^{\prime \prime} \in \mathcal{V}^{p-1}$. By the first item of Corollary 4.2 applied to $V_{p-1}^{\prime \prime}$ and $W_{0}^{c}$ we have $V_{p-1}^{\prime \prime} \subseteq W_{0}$. Since $U_{0}$ is co-connected, by Proposition 3.1 we have that $\partial_{\bullet} U_{0} \cup \partial_{\circ} U_{0}$ is a connected set of vertices of height less or equal to $h\left(\partial_{\circ} W\right)-\delta / 2+1=h\left(\partial_{\bullet} W_{0}\right)-3 p+2$. Therefore $\partial_{\bullet} U_{0} \cup \partial_{\circ} U_{0} \subseteq \mathrm{LC}_{h}^{\left(h\left(\partial_{\bullet} W_{0}\right)-3 p+2\right)+}(u)$ and since $w \notin U_{0}$ we have $U_{0} \subseteq V_{p-1}^{\prime \prime}$ and hence $-n e_{1} \in V_{p-1}^{\prime \prime}$. Since $W_{0}-\Delta \subsetneq U_{0} \subsetneq V_{p-1}^{\prime \prime} \subsetneq W_{0}$ and since $W_{0}$ is of type 0 , we get from Proposition 3.6 that $V_{p-1}^{\prime \prime}$ is of type 0 . By the minimality of $W_{0}$ we get that $0 \notin V_{p-1}^{\prime \prime}$. Thus $V_{p-1}^{\prime \prime} \in \mathcal{V}^{p-1}$, and by the maximality of $V_{0}^{p-1}$ we get that $U_{0} \subsetneq V_{p-1}^{\prime \prime} \subseteq V_{0}^{p-1}$ as required.

For each $0 \leq i \leq p-1$, repeating the proof of Proposition 5.2 with $V_{0}^{i}+j \Delta$ in the role of $V_{j}$ we get that $\Delta$ is a minimal translation of $V_{0}^{i}$, and that, moreover, for every $z \in n \mathbb{Z}^{d}$ and integer $k$,

$$
V_{0}^{i}+z=V_{0}^{i}+k \Delta \text { if and only if } W_{0}+z=W_{0}+k \Delta .
$$

In particular, (6.2) implies that

$$
V_{0}^{i}+n e_{1}=V_{0}^{i}+\ell \Delta \quad \text { for all } 0 \leq i \leq p-1 .
$$

Consequently, by Lemma 3.8,

$$
\left|\pi\left(\partial V_{0}^{i}\right)\right| \geq \ell n^{d-1} \quad \text { for all } 0 \leq i \leq p-1 .
$$

In particular, by the definition (6.1) of $p$ and $\ell$,

$$
\sum_{i=0}^{p-1}\left|\pi\left(\partial V_{0}^{i}\right)\right| \geq p \cdot \ell n^{d-1}=\sigma n^{d-1} .
$$


Aiming to use Proposition 6.1, we proceed to find two vertices on the torus such that the sublevel components of $\pi \circ \Psi_{m}(h)$ between these vertices contain the sets $\pi\left(\partial V_{0}^{i}\right)$ in their boundaries.

Proposition 6.3. Let $u \in \partial_{\bullet} V_{0}^{p-1}$ and $v \in \partial_{\circ} V_{0}^{0}$. Then, for each $0 \leq i \leq p-1$,

$$
V_{0}^{i}=\mathrm{LC}_{h}^{(h(u)+3(p-1-i))+}(u, v)
$$

and

$$
\pi\left(\partial V_{0}^{i}\right) \subseteq \partial \mathrm{LC}_{\pi \circ \Psi_{m}(h)}^{(h(u)+3(p-1-i))+}(\pi(u), \pi(v)) .
$$

Proof. As $u \in \partial_{\bullet} V_{0}^{p-1}$, we have $h(u)=h\left(\partial_{\bullet} W_{0}\right)-3 p+2$ and as $v \in \partial_{\circ} V_{0}^{0}$ we have $h(v)=h\left(\partial_{\bullet} W_{0}\right)$. Denote

$$
A^{i}:=\operatorname{LC}_{h}^{\left(h\left(\partial_{\bullet} W_{0}\right)-3 i-1\right)+}(u, v)=\operatorname{LC}_{h}^{(h(u)+3(p-1-i))+}(u, v), \quad 0 \leq i \leq p-1 .
$$

We show that $V_{0}^{i}=A^{i}$ for all $i$, proving (6.6). First, as $u \in \partial_{\bullet} V_{0}^{p-1}$ and $h(v)>h(u)$, we immediately have that $A^{p-1}=V_{0}^{p-1}$ by Corollary 4.4. Now fix $0 \leq i<p-1$. It follows also that $A^{i} \supseteq V_{0}^{p-1}$. Moreover, as $h\left(\partial_{\bullet} V_{0}^{i}\right)=h\left(\partial_{\bullet} A^{i}\right)$ and, by (6.3), $u \in V_{0}^{i}$, it follows that $\operatorname{LC}_{h}^{h\left(\partial_{\bullet} A^{i}\right)+}(u) \subseteq V_{0}^{i}$. From this, as $v \notin V_{0}^{i}$ by $(6.3)$ and $\left(V_{0}^{i}\right)^{c}$ is connected, we conclude that $A^{i} \subseteq V_{0}^{i}$. Since $A^{i}$ both contains and is contained in sublevel components of type 0 , we conclude from Proposition 3.6 that $A^{i}$ is of type 0 . The minimality of $V_{0}^{i}$ now implies that $V_{0}^{i} \subseteq A^{i}$, leading to the equality $V_{0}^{i}=A^{i}$ that we wanted to prove.

We now proceed to prove (6.7). Fix $0 \leq i \leq p-1$. Let us prove first that

$$
\pi\left(\partial_{\bullet} V_{0}^{i}\right) \subseteq \operatorname{LC}_{\pi \circ \Psi_{m}(h)}^{h\left(\partial_{\bullet} V_{0}^{i}\right)+}(\pi(u)) .
$$

To this end, it suffices to show that

$$
\partial_{\bullet} V_{0}^{i} \subseteq \mathrm{LC}_{\Psi_{m}(h)}^{h\left(\partial_{\bullet} V_{0}^{i}\right)+}(u) .
$$

Denote $B^{i}:=\operatorname{LC}_{h}^{h\left(\partial_{\bullet} V_{0}^{i}\right)+}(u)$. By the definition (5.11) of $\Psi_{m}$ we have (as in (5.14))

$$
\Psi_{m}(h)(w)=h(w) \text { for all } w \in W_{0}^{+} \backslash U_{0}^{-} .
$$

Therefore, as $\partial_{\bullet} V_{0}^{i} \subseteq B^{i}$ by (6.6) and part 4 of Proposition 4.1, as $\partial_{\bullet} V_{0}^{i} \subseteq W_{0}^{+} \backslash U_{0}^{-}$by (6.3) and as $u \in B^{i} \cap\left(W_{0}^{+} \backslash\right.$ $\left.U_{0}^{-}\right)$, the containment (6.9) will follow once we show that $B^{i} \cap\left(W_{0}^{+} \backslash U_{0}^{-}\right)$is connected. To see this, note that as $h\left(\partial_{\bullet} W_{0}\right)>h\left(\partial_{\bullet} V_{0}^{i}\right)$ and $u \in W_{0}$, it follows from the definition of $B^{i}$ that $B^{i} \subseteq W_{0}$. Moreover, as $\partial_{\bullet} U_{0} \cup \partial_{\circ} U_{0}$ is connected by Proposition 3.1 and as $h\left(\partial_{\bullet} V_{0}^{i}\right)>h\left(\partial_{\bullet} U_{0}\right)$, it follows from the definition of $B^{i}$ that if $B^{i} \cap U_{0} \neq \varnothing$ then $\partial_{\bullet} U_{0} \cup \partial_{\circ} U_{0} \subseteq B^{i}$. Since $B^{i}$ is connected by its definition, we conclude that $B^{i} \backslash U_{0}^{-}$is also connected. It follows that $B^{i} \cap\left(W_{0}^{+} \backslash U_{0}^{-}\right)$is connected, as we wanted to show, implying (6.9) and (6.8).

To prove (6.7), it remains to show that

$$
\pi\left(\partial_{\circ} V_{0}^{i}\right) \subseteq \operatorname{LC}_{\pi \circ \Psi_{m}(h)}^{h\left(\partial_{\bullet} V_{0}^{i}\right)+}(\pi(u), \pi(v))^{c} .
$$

Write $C^{i}:=\operatorname{LC}_{\pi \circ \Psi_{m}(h)}^{h\left(\partial_{\bullet} V_{0}^{i}\right)+}(\pi(u))$. As both $v \in W_{0}^{+} \backslash V_{0}^{i}$ and $\partial_{\circ} V_{0}^{i} \subseteq W_{0}^{+} \backslash V_{0}^{i}$ by (6.3), the containment (6.11) is a consequence of

$$
\begin{aligned}
& \pi\left(W_{0}^{+} \backslash V_{0}^{i}\right) \text { is connected, } \\
& C^{i} \cap \pi\left(W_{0}^{+} \backslash V_{0}^{i}\right)=\varnothing .
\end{aligned}
$$


Let us show that $W_{0}^{+} \backslash V_{0}^{i}$ is connected, which will imply (6.12). Indeed, the facts that $V_{0}^{i} \subseteq W_{0}$ by (6.3) and $h\left(\partial_{\circ} W_{0}\right) \geq h\left(\partial_{\bullet} V_{0}^{i}\right)+2$ imply that $\left(V_{0}^{i}\right)^{++} \backslash V_{0}^{i} \subseteq W_{0}^{+} \backslash V_{0}^{i}$. Proposition 3.1 shows that $\left(V_{0}^{i}\right)^{++} \backslash V_{0}^{i}$ is connected. Thus, as $W_{0}^{+}$is connected we obtain that $W_{0}^{+} \backslash V_{0}^{i}$ remains connected.

Let us now prove (6.13). Since, by (6.10) and (6.3), we have the inequalities $\Psi_{m}(h)\left(\partial_{\circ} W_{0}\right)>h\left(\partial_{\bullet} V_{0}^{i}\right)$ and $\Psi_{m}(h)\left(\partial_{\circ} V_{0}^{i}\right)>h\left(\partial_{\bullet} V_{0}^{i}\right)$, it suffices to show that

$$
\pi(u) \notin \pi\left(W_{0}^{+} \backslash V_{0}^{i}\right) .
$$

Suppose, in order to obtain a contradiction, that $u+z \in W_{0}^{+} \backslash V_{0}^{i}$ for some $z \in n \mathbb{Z}^{d}$. Recalling (6.4), let $k$ be the integer satisfying $V_{0}^{i}+z=V_{0}^{i}+k \Delta$ and $W_{0}+z=W_{0}+k \Delta$. Necessarily $k \neq 0$ as otherwise, since $u \in V_{0}^{i}$ by (6.3), also $u+z \in V_{0}^{i}+z=V_{0}^{i}$ and hence $u+z \notin W_{0}^{+} \backslash V_{0}^{i}$. For $k \neq 0$, as $u \in W_{0} \backslash\left(\left(W_{0}-\Delta\right)^{+}\right)$by (6.3), Proposition 5.1 and the fact that $h(u)=h\left(\partial_{\bullet} W_{0}\right)-\frac{\delta}{2}+2>h\left(\partial_{\bullet}\left(W_{0}-\Delta\right)\right)=h\left(\partial_{\bullet} W_{0}\right)-\delta$, it follows that $u+z \in\left(W_{0}+k \Delta\right) \backslash$ $\left(\left(W_{0}-(k-1) \Delta\right)^{+}\right)$. This contradicts our assumption that $u+z \in W_{0}^{+} \backslash V_{0}^{i}$ as $\left(W_{0}+k \Delta\right) \backslash\left(\left(W_{0}-(k-1) \Delta\right)^{+}\right)$is disjoint from $W_{0}^{+} \backslash\left(W_{0}-\Delta\right)$ and $\left(W_{0}-\Delta\right) \subseteq V_{0}^{i}$ by (6.3) and Proposition 5.1. This finishes the proof of (6.13) and hence the proof of the proposition.

We may now deduce Theorem 2.4 in a straightforward manner.

Proof of Theorem 2.4. Let $m \in 6 \mathbb{Z}^{d} \backslash\{\boldsymbol{0}\}$. Assume, without loss of generality (as $\left|\mathrm{QP}_{m}\right|$ does not change when permuting the coordinates of $m$ and replacing $m$ with $-m$ ), that the first coordinate of $m$ is positive and is the largest in absolute value among all coordinates. Recall from (6.1) that for each $h \in \mathrm{QP}_{m}$ we may define $p=p(h)$ and $\ell=\ell(h)$. As both $p(h)$ and $\ell(h)$ are positive integers it follows from (6.1) that $1 \leq p(h) \leq \frac{m_{1}}{6}$. Denote $\mathrm{QP}_{m, p}:=\{h \in$ $\left.\mathrm{QP}_{m}: p(h)=p\right\}$.

Proposition 6.3 together with (6.5) show that for each $h \in \mathrm{QP}_{m, p}$, denoting $r:=\pi \circ \Psi_{m}(h)$, there exist $u, v \in \mathbb{T}_{n}^{d}$ satisfying $r(v)=r(u)+3 p-2$ and $\sum_{j=0}^{3 p-3}\left|\partial \mathrm{LC}_{r}^{(r(u)+j)+}(r(u), r(v))\right| \geq \frac{m_{1}}{6} n^{d-1}$. This allows us to apply Proposition 6.1, using also that $\pi \circ \Psi_{m}$ is one-to-one and that $\left|\operatorname{Hom}\left(\mathbb{T}_{n}^{d}\right)\right|=\left|\mathrm{QP}_{\mathbf{0}}\right|$, to deduce that in high dimensions,

$$
\begin{aligned}
\frac{\left|\mathrm{QP}_{m}\right|}{\left|\mathrm{QP}_{\mathbf{0}}\right|} & =\frac{\left|\left(\pi \circ \Psi_{m}\right)\left(\mathrm{QP}_{m}\right)\right|}{\left|\operatorname{Hom}\left(\mathbb{T}_{n}^{d}\right)\right|}=\frac{\sum_{p=1}^{m_{1} / 6}\left|\left(\pi \circ \Psi_{m}\right)\left(\mathrm{QP}_{m, p}\right)\right|}{\left|\operatorname{Hom}\left(\mathbb{T}_{n}^{d}\right)\right|} \\
& \leq \frac{m_{1}}{6} \exp \left(-\frac{c m_{1} n^{d-1}}{d \log ^{2} d}\right) \leq \exp \left(-\frac{c^{\prime} m_{1} n^{d-1}}{d \log ^{2} d}\right)
\end{aligned}
$$

for some $c, c^{\prime}>0$, as we wanted to show.

\section{Near optimality of the bound}

In this section we prove Proposition 1.3.

Assume that the dimension $d$ is sufficiently large for the following calculations and fix an even integer $n$. Let $f$ be a uniformly chosen proper 3 -coloring of $\mathbb{T}_{n}^{d}$. Define the events

$$
A_{x}:=\left\{f(y)=0 \text { for all } y \in \mathbb{T}_{n}^{d} \text { with } \operatorname{dist}(x, y)=2\right\}, \quad x \in \mathbb{T}_{n}^{d}
$$

The following claim is a consequence of our main theorem, Theorem 1.1.

Lemma 7.1. For each $x \in \mathbb{T}_{n}^{d}$ we have

$$
\mathbb{P}\left(A_{x}\right) \geq \frac{1}{7}
$$


Proof. Observe that Theorem 1.1 and Markov's inequality imply that there exists a constant $c>0$ for which

$$
\mathbb{P}\left(\min _{i \in\{0,1\}} \mathrm{CP}_{i, k}(f) \geq \exp \left(-\frac{c d}{\log ^{2} d}\right)\right) \leq \exp \left(-\frac{c d}{\log ^{2} d}\right), \quad k \in\{0,1,2\} .
$$

By the union bound,

$$
\mathbb{P}(E) \leq 3 \exp \left(-\frac{c d}{\log ^{2} d}\right),
$$

where

$$
E:=\left\{\exists k \in\{0,1,2\}, \min _{i \in\{0,1\}} \mathrm{CP}_{i, k}(f) \geq \exp \left(-\frac{c d}{\log ^{2} d}\right)\right\} .
$$

As the coloring $f$ is proper, on the event $E^{c}$ we necessarily have some random $k_{0} \in\{0,1,2\}$ and $i_{0} \in\{0,1\}$ such that

$$
\mathrm{CP}_{i_{0}, k_{0}}(f) \geq 1-2 \exp \left(-\frac{c d}{\log ^{2} d}\right) .
$$

By the homogeneity of the torus, it follows that for each $y \in V^{i_{0}}$,

$$
\mathbb{P}\left(f(y) \neq k_{0} \mid E^{c}, i_{0}\right) \leq 2 \exp \left(-\frac{c d}{\log ^{2} d}\right) .
$$

We conclude that for each $x \in V^{i_{0}}$,

$$
\mathbb{P}\left(f(y)=k_{0} \text { for all } y \in \mathbb{T}_{n}^{d} \text { with } \operatorname{dist}(x, y)=2 \mid E^{c}, i_{0}\right) \geq 1-8 d^{2} \exp \left(-\frac{c d}{\log ^{2} d}\right) .
$$

Using the homogeneity of the torus again and symmetry between the 3 colors, for each $x \in \mathbb{T}_{n}^{d}$,

$$
\mathbb{P}\left(A_{x} \mid E^{c}\right) \geq \frac{1}{6}\left(1-8 d^{2} \exp \left(-\frac{c d}{\log ^{2} d}\right)\right),
$$

from which the lemma follows for sufficiently large $d$ using (7.1).

We note for later use that for each $x \in \mathbb{T}_{n}^{d}$, by the domain Markov property,

$$
\mathbb{P}\left(f(x)=1 \mid A_{x}\right)=\frac{1}{2^{2 d}+2} \quad \text { and } \quad \mathbb{P}\left(f(x+e)=1 \mid A_{x}\right)=\frac{1}{2},
$$

where $e$ is a standard basis vector.

Let $T \subset V^{0}$ be such that for any two distinct $x_{1}, x_{2} \in T$ we have $\operatorname{dist}\left(x_{1}, x_{2}\right) \geq 4$ and $|T| \geq \frac{\left|V^{0}\right|}{5 d^{2}}$. Such a set may be constructed greedily as for any $x \in V^{0},\left|\left\{y \in V^{0}: \operatorname{dist}(x, y) \leq 3\right\}\right| \leq 5 d^{2}$. Let $T_{0} \subset T$ be the collection of $x \in T$ for which the event $A_{x}$ occurs and write $S=\left|T_{0}\right|$. We have $\mathbb{E}(S) \geq \frac{1}{7}|T|$ by Lemma 7.1, whence by Markov's inequality for $|T|-S$ we obtain

$$
\mathbb{P}\left(S>\frac{1}{8}|T|\right)=1-\mathbb{P}\left(|T|-S \geq \frac{7}{8}|T|\right) \geq 1-\frac{8}{7} \frac{|T|-\mathbb{E}(S)}{|T|} \geq \frac{1}{49} .
$$

For each $x \in \mathbb{T}_{n}^{d}$ define the 1-ball around $x$ by

$$
B_{x}:=\left\{y \in \mathbb{T}_{n}^{d}: \operatorname{dist}(x, y) \leq 1\right\} .
$$


Let $\mathcal{F}_{T}$ be the sigma algebra generated by the values $f(y)$, where $y$ ranges over all vertices in $\mathbb{T}_{n}^{d}$ satisfying that $\operatorname{dist}(x, y)=2$ for some $x \in T$. Observe that $T_{0}$ is measurable with respect to $\mathcal{F}_{T}$. Observe further that conditioned on $\mathcal{F}_{T}$, by the domain Markov property, the values that $f$ takes on each $B_{x}, x \in T_{0}$, are uniformly sampled 3-colorings of $B_{x}$ with zero boundary conditions on $\partial_{\circ} B_{x}$, and these colorings are independent between the different $x \in T_{0}$. Fix an arbitrary (measurable with respect to $\mathcal{F}_{T}$ ) partition $T_{0}^{0} \uplus T_{0}^{1}=T_{0}$ with $\left|T_{0}^{0}\right|=\left\lfloor\frac{S}{2}\right\rfloor$ and $\left|T_{0}^{1}\right|=\left\lceil\frac{S}{2}\right\rceil$. We then have that, conditioned on $\mathcal{F}_{T}$,

$$
\min _{i \in\{0,1\}} \mathrm{CP}_{i, 1}(f) \geq \frac{\min \left(\left|\left\{x \in T_{0}^{0}: f(x)=1\right\}\right|,\left|\left\{x \in T_{0}^{1}: f\left(x+e_{1}\right)=1\right\}\right|\right)}{\left|V^{0}\right|}=\frac{\min (X, Y)}{\left|V^{0}\right|},
$$

where $X, Y$ are independent binomial random variables satisfying, by (7.2), $X \sim \operatorname{Bin}\left(\left\lfloor\frac{S}{2}\right\rfloor, \frac{1}{2^{2 d}+2}\right)$ and $Y \sim$ $\operatorname{Bin}\left(\left\lceil\frac{S}{2}\right\rceil, \frac{1}{2}\right)$. The fact that $S$ is measurable with respect to $\mathcal{F}_{T}$, together with (7.3), now allows to conclude that

$$
\mathbb{E}\left(\min _{i \in\{0,1\}} \mathrm{CP}_{i, 1}(f)\right) \geq \frac{c^{\prime}}{d^{2}} 2^{-2 d},
$$

for some $c^{\prime}>0$. As the color $k=1$ is arbitrary, this concludes the proof of the proposition.

\section{Remarks and open problems}

In this section we discuss a few open problems and make a remark.

1. (Tori with odd side length) In this work we consider a uniformly sampled proper 3-coloring of a high-dimensional discrete torus with even side length. Our main result is that for such a coloring, with high probability, one of the two bipartition classes is dominated by a single color. How will this result change if we take $n$, the side length of the torus, to be odd? since tori with odd side length are no longer bipartite, some change must occur. We expect that in this situation, we will find in a typical coloring three 'pure phase' regions. Each of these regions will have a distinct dominant color coloring one of its bipartition classes while the two remaining colors equally dominate the other bipartition class. Every two regions will be separated by a single long odd interface (of size roughly $n^{d-1}$ ), and the vertices on each side of the interface will be colored by the dominant color of their region.

2. (Positive temperature) In physical terminology, a uniformly chosen proper 3-coloring is the zero-temperature case of the antiferromagnetic 3-state Potts model. The positive temperature version of this model is defined as follows. A 3-coloring $f$, not necessarily proper, of the underlying graph is sampled with probability proportional to $\exp (-\beta H(f))$, where $\beta>0$ is a parameter proportional to the inverse temperature and $H(f)$ is the number of edges $(u, v)$ for which $f(u)=f(v)$. We expect that the analog of Theorem 1.1 continues to hold when the temperature is small, but positive (that is, when $\beta$ is sufficiently large). Proving this is complicated by the fact that non-proper 3-colorings are no longer related to height functions.

3. (Larger number of colors) As explained in Section 1.3, it is expected that Theorem 1.1 has a natural extension to proper colorings of the torus with more than 3 colors. Specifically, that for each $q$ there is some $d_{0}(q)$ such that if $d \geq d_{0}(q)$ then a typical proper $q$-coloring of $\mathbb{T}_{n}^{d}$ has the property that the $q$ colors split into two sets of sizes $\lfloor q / 2\rfloor$ and $\lceil q / 2\rceil$ with each bipartition class dominated by colors from one of the two sets. Proving this is wide open even for the case $q=4$. A result of Vigoda [23] implies that $d_{0}(q) \geq \frac{3}{11} q$. In [4, Conjecture 5.3] it is conjectured that $d_{0}(q)=q / 2$, at least in the sense that certain "long range influences" exist if and only if $d \geq q / 2$. However, any result showing that $d_{0}(q)<\infty$ will constitute a major advance.

We end with the following remark. Our work extends certain results from [18]. The results in [18] were proven in greater generality than simply for the torus $\mathbb{T}_{n}^{d}$. There, also tori with non-equal side lengths were considered, of the form $\mathbb{T}_{n_{1}}^{1} \times \mathbb{T}_{n_{2}}^{1} \times \cdots \times \mathbb{T}_{n_{d}}^{1}$. These include, in particular, "two-dimensional" tori of the form $\mathbb{T}_{n}^{2} \times \mathbb{T}_{2}^{d}$ for $d$ a fixed large constant. In our work, for simplicity, we considered only the case of the torus $\mathbb{T}_{n}^{d}$. However, it seems that our arguments can be adapted with no difficulty to the more general tori for which results were obtained in [18]. 


\section{Acknowledgements}

We thank Eugenii Shustin for useful discussions on the relation between our work and algebraic topology. We also thank Naomi Feldheim, Wojciech Samotij and Yinon Spinka for many useful comments which greatly improved the quality of the paper. We are also grateful to the anonymous referees whose insightful comments significantly helped to improve the presentation.

\section{References}

[1] C. Boutillier and B. de Tilière. Loop statistics in the toroidal honeycomb dimer model. Ann. Probab. 37 (5) (2009) 1747-1777. MR2561433

[2] G. R. Brightwell, O. Häggström and P. Winkler. Nonmonotonic behavior in hard-core and Widom-Rowlinson models. J. Stat. Phys. 94 (3-4) (1999) 415-435. MR1675359

[3] R. L. Dobrušin. The problem of uniqueness of a gibbsian random field and the problem of phase transitions. Funkcional. Anal. i Priložen. 2 (4) (1968) 44-57.

[4] J. Engbers and D. Galvin. H-coloring tori. J. Combin. Theory Ser. B 102 (5) (2012) 1110-1133. MR2959393

[5] D. Galvin. On homomorphisms from the Hamming cube to Z. Israel J. Math. 138 (2003) 189-213. MR2031957

[6] D. Galvin and J. Kahn. On phase transition in the hard-core model on $\mathbb{Z}^{d}$. Combin. Probab. Comput. 13 (2) (2004) 137-164. MR2047233

[7] D. Galvin, J. Kahn, D. Randall and G. Sorkin. Phase coexistence and torpid mixing in the 3-coloring model on $Z^{d}$. SIAM J. Discrete Math. 29 (3) (2015) 1223-1244. MR3374637

[8] D. Galvin and D. Randall. Torpid mixing of local Markov chains on 3-colorings of the discrete torus. In Proc. ACM-SIAM SODA 376-384. ACM, New York, 2007. MR2482863

[9] D. Galvin and P. Tetali. On weighted graph homomorphisms. In Graphs, Morphisms and Statistical Physics 97-104. DIMACS Ser. Discrete Math. Theoret. Comput. Sci. 63. Amer. Math. Soc., Providence, RI, 2004. MR2056231

[10] Y. Huang, K. Chen, Y. Deng, J. L. Jacobsen, R. Kotecký, J. Salas, A. D. Sokal and J. M. Swart. Two-dimensional Potts antiferromagnets with a phase transition at arbitrarily large $q$. Phys. Rev. E 87 (1), 012136 (2013).

[11] J. Kahn. Range of cube-indexed random walk. Israel J. Math. 124 (2001) 189-201. MR1856513

[12] J. Kahn. An entropy approach to the hard-core model on bipartite graphs. Combin. Probab. Comput. 10 (3) (2001) 219-237. MR1841642

[13] R. Kenyon Lectures on Dimers, in Statistical Mechanics. IAS/Park City Math. Ser. 16, 191-230. Amer. Math. Soc., Providence, RI, 2009. MR2523460

[14] R. Kotecký. Long-range order for antiferromagnetic Potts models. Phys. Rev. B 31 (1985) 3088-3092.

[15] R. Kotecký, A. D. Sokal and J. M. Swart. Entropy-driven phase transition in low-temperature antiferromagnetic Potts models. Comm. Math. Phys. 330 (3) (2014) 1339-1394. MR3227515

[16] E. H. Lieb. The residual entropy of square ice. Phys. Rev. 162 (1) (1967) 162-172.

[17] T. Meyerovitch and R. Pavlov. On independence and entropy for high-dimensional isotropic subshifts. Proc. Lond. Math. Soc. (3) 109 (4) (2014) 921-945. MR3273488

[18] R. Peled. High-dimensional Lipschitz functions are typically flat. To appear in Ann. Prob. Preprint. Available at arXiv:1005.4636.

[19] R. Peled and W. Samotij. Odd cutsets and the hard-core model on $\mathbb{Z}^{d}$. Ann. I. H. Poincaré (B) 50 (3) (2014) 975-998. MR3224296

[20] R. Peled, W. Samotij and A. Yehudayoff. Lipschitz functions on expanders are typically flat. Combin. Probab. Comput. 22 (4) (2013) 566-591. MR3073490

[21] R. Peled, W. Samotij and A. Yehudayoff. Grounded Lipschitz functions on trees are typically flat. Electron. Commun. Probab. 18 (55) (2013) 1-9. MR3078018

[22] Á. Timár. Boundary-connectivity via graph theory. Proc. Amer. Math. Soc. 141 (2) (2013) 475-480. MR2996951

[23] E. Vigoda. Improved bounds for sampling colorings. J. Math. Phys. 41 (3) (2000) 1555-1569. MR1757969 